\title{
Resource Allocation for Energy \\ Harvesting Communication Systems over \\ FADING CHANNELS \\ by
}

\author{
Mohammed Abubakor Baljon \\ Master of Engineering, Dalhousie University, 2012 \\ Bachelor of Science, King Abdulaziz University, 2007 \\ A dissertation \\ presented to Ryerson University \\ in partial fulfillment of the \\ requirements for the degree of \\ Doctor of Philosophy \\ in the Program of
}

Electrical and Computer Engineering

Toronto, Ontario, Canada, 2019

(C)Mohammed Abubakor Baljon 2019 


\section{AUTHOR'S DECLARATION FOR ELECTRONIC SUBMISSION OF A DISSERTATION}

I hereby declare that I am the sole author of this dissertation. This is a true copy of the thesis, including any required final revisions, as accepted by my examiners.

I authorize Ryerson University to lend this dissertation to other institutions or individuals for the purpose of scholarly research.

I further authorize Ryerson University to reproduce this dissertation by photocopying or by other means, in total or in part, at the request of other institutions or individuals for the purpose of scholarly research.

I understand that my dissertation may be made electronically available to the public. 
Resource Allocation for Energy Harvesting Communication Systems over Fading Channels

Doctor of Philosophy 2019

Mohammed Abubakor Baljon

Electrical and Computer Engineering

Ryerson University

\begin{abstract}
Abstract: Energy Harvesting (EH) is an emerging communications paradigm to defeat the limitation of network longevity by recharging the nodes by harvesting energy from the environment. The Energy Harvesting Network (EHN) requires a stable and efficient power control scheme like other conventional communication systems. It is more complicated than conventional communication networks, in that it should not only consider the quality of service requirements of the network but also adapt to the randomness of the energy arrival. In this thesis, several optimal offline and online resource allocation strategies for point-to-point and two-hop EH communication networks over wireless fading channels are investigated.

As a first step, the RGWF (Recursive Geometric Water-filling) algorithm is introduced, which provides an optimal offline transmission policy for a point-to-point EH communication system. Next, a network composed of a source, a relay, and a destination, where the source is an EH node is considered. Joint time scheduling and power allocation problems are formulated to maximize the network throughput by considering conventional and
\end{abstract}


bufferaided link adaptive relaying protocols. Based on the modified RGWF algorithm, the joint power allocation and transmission time scheduling problem are decoupled, and efficient offline schemes are proposed for a two-hop wireless network for delay-tolerant and delay sensitive applications. In the second part, the aim is to obtain the optimal transmission policy that maximizes the average total throughput of a point-to-point EH communication system with low and high data arrival rate in an online manner. The solution is obtained using dynamic programming by casting the proposed problem as a semi-Markov decision process (SMDP). In a delay-tolerant approach with high data rate, a cross-layer adaptation is considered, where the proposed policy chooses modulation constellation for EH networks dynamically, depending on battery state, data buffer state in addition to channel state. The proposed SMDP-based dynamic programming approach has proven to be dynamically adaptive to the change of the channel and/or buffer states that optimally satisfy the BER requirements at the physical layer, and the overflow requirements at the data-link layer. 


\section{Acknowledgements}

I would like to express my deepest appreciation and sincere gratitude to my supervisor Professor Lian Zhao for her continuous guidance, support, and encouragement. Her valuable advice and extended knowledge was a great help during my $\mathrm{PhD}$ journey. The experience that I gained while working under her supervision has greatly developed my academic and research skills. I am also grateful to my friends, teachers and classmates.

My special thanks go to all the members of the examination committee: Dr. Anpalagan, Dr. Liao, Dr. Ma, and Dr. Wang (in alphabetical order).

Special thanks and a deep gratitude to the Saudi Cultural Bureau in Canada and the Ministry of High Education in Saudi Arabia for their financial and academic support throughout my advanced education.

Finally, I am truly indebted to my family members for their endless support, encouragement, and prayers. 


\section{Table of Contents}

Declaration ............................. ii

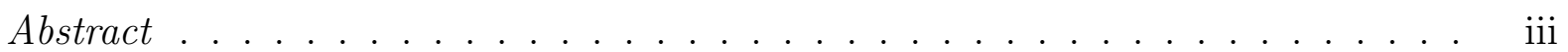

Acknowledgements ........................

List of Tables . . . . . . . . . . . . . . . . . . . $\mathrm{x}$

List of Figures . . . . . . . . . . . . . . . . . . . xi

List of Appendices . . . . . . . . . . . . . . . . . . . xiii

1 Introduction $\quad 1$

1.1 Background and Motivation . . . . . . . . . . . . . . . . 1

1.2 Energy Harvesting in Wireless Communication Systems _. . . . . . . . 4

1.2.1 Characteristics of EH Sources . . . . . . . . . . . . . 5

$1.2 .2 \quad$ Storage Capacity . . . . . . . . . . . . . . . . . . 7

1.3 Literature Review . . . . . . . . . . . . . . . . . . . . . . 8

1.4 Contributions . . . . . . . . . . . . . . . . . . . . . . 10

1.5 Thesis Organization . . . . . . . . . . . . . . . . . . . 12

2 Resource Allocation Problems and General Tools $\quad 15$

2.1 Wireless System Model . . . . . . . . . . . . . . . . . . . . . . 15

2.2 Resource Allocation Approaches . . . . . . . . . . . . . . . . . . . 16

2.3 Resource Allocation and Service Requirements . . . . . . . . . . . . . . 17

2.3 .1 Objective Function . . . . . . . . . . . . . . . . 18 
2.3 .2 Constraints . . . . . . . . . . . . . . . . . . . . . . . . . . . . 19

2.4 Optimization Framework and General Tools . . . . . . . . . . . . . . 21

2.4 .1 Single-hop Conventional Communication Systems . . . . . . . . . . 22

2.4 .2 Single-hop EH Communication Systems: Offline Setting . . . . . . . . 25

2.4 .3 Single-hop EH Communication Systems: Online Setting . . . . . . . . 29

2.5 Chapter Summary . . . . . . . . . . . . . . . . . . . . . . 31

\section{Resource Allocation for Two-Hop Communication with Energy Harvesting Constraints Over Fading Channels 32}

3.1 Introduction . . . . . . . . . . . . . . . . . . . . . . . . 32

3.2 System Model . . . . . . . . . . . . . . . . . . . . . . . . . . 34

3.2 .1 Model Description . . . . . . . . . . . . . . . . . . 34

$3.2 .2 \quad$ RGWF-EH Profile . . . . . . . . . . . . . . . . . . . . 35

3.2 .3 Transmission policy . . . . . . . . . . . . . . . . . 36

3.3 Throughput Maximization Problem for the DS case . . . . . . . . . . 38

3.3 .1 Optimal Resource Allocation . . . . . . . . . . . . . . . . . . . . . 39

3.3 .2 Sub-optimal Resource Allocation . . . . . . . . . . . . . . . 40

3.3.3 Pre-defined Data Rates (PDDR) Based . . . . . . . . . . . . . . . 41

3.4 Throughput Maximization Problem for the DT Case . . . . . . . . . . 43

3.4 .1 Optimal Resource Allocation . . . . . . . . . . . . . . . . . . 44

$3.4 .2 \quad$ Sub-Optimal Resource Allocation . . . . . . . . . . . . . . . . 44

3.4.3 Relay In Demand using Average Fading (RID-AF) Based . . . . . . . 46

3.5 Simulation Results . . . . . . . . . . . . . . . . . 46

3.5.1 Performance of RGWF algorithm for a point-to-point communication 48

3.5.2 Resource Allocation Schemes for Delay-Sensitive Two-hop Communi-

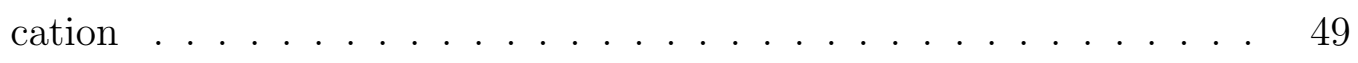

3.5.3 Resource Allocation Schemes for Delay Tolerant Two-hop Communi-

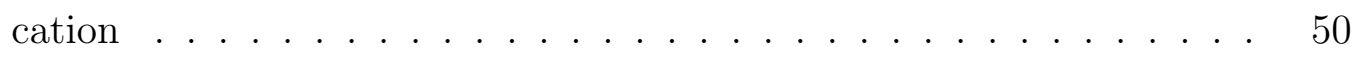


3.6 Chapter Summary . . . . . . . . . . . . . . . . . . . . . . 52

4 SMDP-Based Resource Allocation for Wireless Networks with Energy Harvesting Constraints $\quad 54$

4.1 Introduction . . . . . . . . . . . . . . . . . . . . 54

4.2 System Model . . . . . . . . . . . . . . . . . . . . . . 56

4.2 .1 Model Description . . . . . . . . . . . . . . . . 56

4.2 .2 Problem Formulation . . . . . . . . . . . . . . . . . . . . . . 58

4.3 SMDP Formulation Of EH Technology . . . . . . . . . . . . . . 59

4.3 .1 System States . . . . . . . . . . . . . . . . . 60

4.3 .2 Set of Actions . . . . . . . . . . . . . . . . . . . 60

4.3 .3 Reward Model . . . . . . . . . . . . . . . . . . . . . . . . . . . 61

4.3 .4 Sojourn Time . . . . . . . . . . . . . . . . . . . . . . . . 62

4.3 .5 Transition Probability . . . . . . . . . . . . . . 63

4.4 Energy Allocation Scheme By SMDP . . . . . . . . . . . . . . . 65

4.5 Performance Evaluation . . . . . . . . . . . . . . . 66

4.6 Chapter Summary . . . . . . . . . . . . . . . . . . . . . . 68

5 Cross-layer Adaptive Transmission Techniques for EH Systems over Fading $\begin{array}{ll}\text { Channels } & \mathbf{7 0}\end{array}$

5.1 Introduction . . . . . . . . . . . . . . . . . 70

5.2 System Model . . . . . . . . . . . . . . . . . . . . . . . . 75

5.2 .1 Model Description . . . . . . . . . . . . . . . . 75

5.2 .2 Channel Model . . . . . . . . . . . . . . . . . . . . . . . . . . 77

5.2 .3 Battery Model . . . . . . . . . . . . . . . . . . . . . . 78

5.2 .4 Data Buffer Model . . . . . . . . . . . . . . . . . 78

5.3 SMDP Formulation of EH Cross-layer Adaptive Transmission $\ldots . . .79$

5.3 .1 Problem Description . . . . . . . . . . . . . . . . . . 79 
5.3.2 Problem Formulation and Policy . . . . . . . . . . . . 86

5.4 Solution Techniques . . . . . . . . . . . . . . . . . 87

5.4.1 Cross-Layer and Policy Evaluation . . . . . . . . . . . 88

5.5 Performance Evaluation . . . . . . . . . . . . . . . . . . . . 90

5.6 Chapter Summary . . . . . . . . . . . . . . . . . . 94

6 Conclusions $\quad 95$

6.1 Conclusion . . . . . . . . . . . . . . . . . . . 95

$\begin{array}{ll}\text { References } & 111\end{array}$ 


\section{List of Tables}

1.1 Examples of EH sources . . . . . . . . . . . . . . . 5

4.1 List of Notation . . . . . . . . . . . . . . . . . . . . . . 58

5.1 List of Notation . . . . . . . . . . . . . . . . . . 76 


\section{List of Figures}

2.1 Fill the incoming energy until the next energy arrival . . . . . . . . . . . . 27

2.2 Equalizing the water level among the epochs . . . . . . . . . . . . . 27

3.1 System model, $K=7$ epochs in $[0, T]$ with (a) EH source, i.e., random energy arrivals $\left(E_{i n}\right)$, has a time-variant channel, and (b) non-EH relay, i.e., relay powered by battery, experiences an AWGN channel. . . . . . . . . . . . . . . . . 35

3.2 (a) Optimum power allocation for a single-hop with EH constraint over a fading channel using RGWF. (b) RGWF-EH profile. . . . . . . . . . . . . . . . . . . . 36

3.3 Flow chart of RID-AF algorithm. . . . . . . . . . . . . . . . . 47

3.4 Simulation results of the throughput versus the source EH rate for a single-hop over a Rayleigh channel with $\bar{m}=5 . \quad$. . . . . . . . . . . . . . . . . . . . . . . . 49

3.5 Simulation results of the throughput versus the source EH rate for a non-delay tolerant two-hop network under Rayleigh fading with $\bar{m}=5 . \quad$. . . . . . . . . . . 51

3.6 Simulation results of the throughput versus the source EH rate for a delay tolerant two-hop network over a Rayleigh channel with $\bar{m}=5 \ldots$. . . . . . . . . . . . . . 52

3.7 Average throughput versus $\kappa$ with $\left(P_{o}^{E H}=[5,8,10] \mathrm{mW}\right)$ under Rayleigh fading

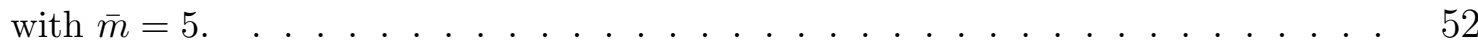

4.1 System block diagram. . . . . . . . . . . . . . . . . . . . . . . . . . . 57

4.2 Average probability of action under various $\lambda_{c} \ldots \ldots \ldots$. . . . . . . . 67

4.3 Overall reward under various $\lambda_{c} \ldots \ldots \ldots$. . . . . . . . . . . . . 68 
4.4 Blocking probability under various $\lambda_{c} \ldots \ldots \ldots$. . . . . . . . . . 69

5.1 System block diagram. . . . . . . . . . . . . . . . . . . . . 75

5.2 Effect of average buffer delay on the throughput among different schemes. . . 91

5.3 Effect of average packet arrival rate on the throughput and the overflow probability rate among different schemes. . . . . . . . . . . . . . . . 92

5.4 Effect of average harvested energy on the throughput for the proposed scheme among various average buffer delays. . . . . . . . . . . . . . . 


\section{List of Appendices}




\section{Chapter 1}

\section{Introduction}

\subsection{Background and Motivation}

With increasing demand and explosive growth in wireless communications in recent years, energy conservation has become more desirable to help reducing the world's energy consumption. According to the International Energy Agency [1], due to tremendous demand for energy in all areas, by 2050, investments in energy preservation could decrease the waste of energy by one-third. Green communication is the key solution to many problems related to the wasted energy due to radio transmissions [2]. Green communication can be defined as the practice of utilizing the energy that is harvsted from the environment wisely by selecting energy-efficient communications technologies. In fact, scavenging ambient energy and utilizing the available energy wisely leads to maximize the overall network throughput $[3,4]$.

Today's number of communication devices, such as smartphones and wireless sensor networks will be doubled or tripled by 2050 [1]. Therefore, the last decade witnessed considerable interest in investigating and proposing more efficient and economical ways to allocate power resources. Besides saving energy, Green communication can solve the $\mathrm{CO} 2$ emission threat that results from the increasing energy consumption in wireless networks. For this reason, a number of countries, organizations and institutions have signed agreements to reduce en- 
ergy consumption by various percentages. By 2020, for instance, the European Union has aimed to reduce greenhouse gas emmissions, the government of China has promised to reduce pollution by 10 percent, and Vodafone Group has targeted to reduce its CO2 emissions by $2020[5][6]$.

Another advantage of utilizing green communication is maximizing the radio communication tasks' lifetime because of its renewability feature. Operation of traditional communication systems cannot surpass the battery size or control the constraints of the power supply. In contrast, nodes with energy harvesting capability in wireless communication systems are able to harvest energy from the renewable sources of their surrounding environment, convert it to electrical energy, and utilize the converted energy in carrying out their functions.. Therefore, EH node technology is a promising technique to overcome the limitation of network longevity as shown in [7-9].

Although all the features of EH wireless networks, such as prolonging network longevity and saving the environment from $\mathrm{CO} 2$ emissions threats, some particular challenges should be considered and a new design dimension should be established. The main challenging of EH technology is the time-varying energy harvesting [10] and the limited amount of energy available [11], which means that satisfactory communication performance is difficult to guarantee. Thus, much effort has been spent on enhancing EH network performance in the literature (e.g., [12-17]). It is revealed that in order to develop efficient transmission policies for EH wireless networks, the random arrivals and the low rate of harvested energy should be addressed by considering the $\mathrm{EH}$ profile that characterizes the $\mathrm{EH}$ capacity of the EH nodes. It is concluded that even when the wireless fading channels remain unchanged, the transmission power should adapt to the time-varying energy arrivals, which adds more challenges to EH wireless networks. Moreover, cooperative relays are used in order to expand the network size geographically, thereby increasing the communication transmission range. Besides multi-hop transmission as it is used to expand the covering transmission area, it also has been approved to maximize the overall throughput of the network [18,19]. Buffer- 
aided relaying is a fundamental concept at the physical layer for many wireless networks, i.e. cellular and ad hoc, etc., and they have been well investigated over the last decade. Intermediate nodes can relay the source information to the destination using minimum power. As a result, energy efficiency is obtained and the high risk of potential interference is avoided. Motivated by these features, deploying relays on EH communication systems is a significant strategy and plays an important role in improving the EH network performance.

On the other hand, as a result of employing data buffering, queuing delay must be considered when developing resource allocation schemes [20-22]. It is known that modern wireless systems must support various delay constraints including delay and non-delay tolerant constraints by providing delay QoS guarantees through efficient resource allocation schemes. Real-time applications are an example of the non-delay tolerant approach, which is classified under hard-deadline constraints, such as online games, real-time audio and video, and intelligent support systems etc. [23,24]. In contrast, other applications including traditional Internet services, such as file transfers, email exchanges, and web browsing, can tolerate some delays but are limited to expect good (average) delay performance. Nevertheless, a modern energy-constrained wireless communication system are subject to time-varying channel conditions and stochastic traffic loads, which makes it very hard for them to provide the necessary QoS to support latency-sensitive applications. This is more challenging by the introduction of wireless nodes powered by energy harvested from the environment, such as solar, motion, thermal energy, light, or RF energy. Although energy harvesting nodes can operate autonomously in remote areas without require to change batteries or access to power lines, the stochastic nature of harvested energy sources poses new challenges in power management, transmission power allocation, and transmission scheduling.

Motivated by the above discussions, this thesis studies novel resource allocation schemes for practical point-to-point and two-hop EH communication systems, where a source does all the optimization part and a receiver is a base station, which is operated by conventional power, to support delay-tolerant and delay-sensitive applications with the objective of max- 
imizing network throughput and longevity while minimizing energy cost. In the considered systems, to overcome the fading nature of wireless channels, the EH nodes use buffers to store the data arrivals, optimally allocate resources, and adapt transmissions to the instantaneous channel conditions and EH profile to enhance the throughput. It also considers the case when the source is equipped with finite-size buffers with different QoS (i.e., delay, packet-dropping rate, packet error rate, bit error rate, etc.) requirements, and cross-layer optimization is investigated, where physical-layer parameters are combined with data-link/higher-layer parameters. These parameters are adapted to channel conditions, battery states, and buffer occupancy to minimize delay, BER, overflow rate, etc., and to maximize throughput. Since these objectives are difficult to manage, we consider a tradeoff between them and find the optimal policy by forming the problem as a semi-Markov decision process (SMDP) and solving the problem with dynamic programming technique.

\subsection{Energy Harvesting in Wireless Communication Sys- tems}

In traditional wireless communication system, transceiver nodes are connected to the power grid or equipped with pre-charged batteries. However, or some applications, connecting the transceiver nodes to the power grid is not possible, while replacing or recharging the batteries is impractical. An alternative solution to tackle this issue is energy harvesting (EH), where wireless EH nodes harvest energy from their surrounding environment, convert it to electrical energy using a corresponding conversion mechanism. Then, the converted electrical energy can be utilized for many purposes such as sensing, signal processing, and/or transmission. Hence, network with EH capability provides a way of operating with an infinite lifetime.

On the other hands, power allocation for $\mathrm{EH}$ transceiver is subject to EH constraints, where each transmitter is constrained to utilize at most the amount of currently available stored energy at each interval even though the transmitter may harvest more energy in the 
Table 1.1: Examples of EH sources

\begin{tabular}{|l|l|l|}
\hline EH source & EH arrival & Harvested power \\
\hline Thermal energy (Human) & Predictable & $0.3 \mathrm{~mW}$ \\
Thermal energy (Industrial) & Predictable & $1-10 \mathrm{~mW}$ \\
Wind energy (turbine) & Unpredictable & $1 \mathrm{~W}$ \\
Wind energy (small-scale) & Unpredictable & $7,5-55 \mathrm{~mW}$ \\
Light energy (Outdoor) & Predictable & $10 \mathrm{~mW} / \mathrm{cm}^{2}$ \\
Light energy (Indoor) & Predictable & $10 \mu W / \mathrm{cm}^{2}$ \\
Vibration energy (human motion) & Predictable & $4 \mathrm{~mW}$ \\
Vibration energy (machines) & Predictable & $1 \mathrm{~W}$ \\
RF transmitter with 4W & Unpredictable & $189 \mu$ Wat $5 \mathrm{~m}$ \\
Powercast transmitter with 3W & Unpredictable & $5.5 \mu W$ at $15 \mathrm{~m}$ \\
\hline
\end{tabular}

future. Therefore, the characteristics of the energy sources, which are represented as the harvested amounts of energy and its arrival time, have a direct influence on the transmission policy design to be either online or offline. For instance, a source of energy can be either controllable or non-controllable; and non-controllable energy can be also divided predictable (offline) or unpredictable (online) transmission strategy.

\subsubsection{Characteristics of EH Sources}

As mentioned earlier, The characteristics of the energy sources directly influences the transmission policy design to be either online or offline. In the following lines, the characteristics of the different energy sources and their performance are presented, which are summarized in Table (1.1).

Thermal energy: An approach, such as Seebeck effect is utilzed to convert the thermal energy into electrical energy [25]. Thermal energy can be harvested when the node is exposed to temperature gradients. One end of the Thermoelectric generator should be placed on the heat source and the other end on a colder source. An example of thermal energy harvesting sources is human bodies ( [26], [27], [28]), room heater [29], CPU heatsinks [30] etc. As a matter of fact, thermal energy is uncontrollable but predictable.

Wind energy: A wind turbine is a well-known source of energy that harvests energy 
from air flows and supplys heavy duty applications, such as power grids, cellular base stations, etc. For a large-scale wind EH system, the Windlab Junior turbine ( [31], [32]) can generate power with $1 \mathrm{Watt}$ at a speed of $2000 \mathrm{rpm}$. Inspired by largescale systems, smallscale wind EH systems are developed. For example, a wind EH turbine is developed in [33], which provides a output power between [7.5 - 55] $\mathrm{mW}$.

Light energy: Light is the most common source of energy with their two well-known types: solar radiation or artificial light [34]. There exist important implementations of solar EH nodes in wireless communications $[35,36]$. Practically, light energy is captured in a solar panel and then converted into electrical energy by using the photovoltaic effect. The output power of solar energy in outdoor and indoor surfaces are $10 \mathrm{~mW} / \mathrm{cm}^{2}$ and $10 \mu \mathrm{W} / \mathrm{cm}^{2}$, respectively [26].

Vibration and Motion energy: Vibrations are energy sources for EH nodes that occur in daily human activity or industry. Electrical energy can be harvested from vibration by many transducers, such as piezoelectric generators or electrostatic and electromagnetic converters [37]. An extensive review of vibration energy harvest leveraging piezoelectric effect is presented in [38]. Vibrations generated from road traffic [39], factory machines [40] and human motions such as footsteps [41], [42] are explored as the source of energy. Researchers in [11] measured the output power of daily human activities and they found that the average harvested power that can be generated from human motion is $4 m W$. On the other hand, vibration that is released from machines can produce energy up to $1 W$ [43].

RF energy: The energy from RF electromagnetic waves has been approved to charge $\mathrm{EH}$ nodes from the far-field electromagnetic radiation in the RF band. Usually, this band ranges from $3 \mathrm{KHz}$ to $300 \mathrm{GHz}$, where most of the energy harvesting research activities are focused on TV [44], Cellular ( [45], [46]), and ISM ( [47], [48], [49]) bands. Authors in [50] shows an example of Wireless Local Area Network (WLAN) powers EH nodes. It shows that the amount of harvested energy that can be obtained from the RF waves relies on RF wavelength $(\lambda)$, transmitting power $(p)$, and the distance between power source and 
the EH node $(d)$. However, the main factor that controls the amount of harvested energy is the distance. Authors in [51] showed that the Powercast transmitter with $3 \mathrm{~W}$ transmit power in $915 \mathrm{MHZ}$ band generates $189 \mu \mathrm{W}$ at $5 m$ distance, whereas RF transmitter with 4 $W$ transmit power in the $928 \mathrm{MHZ}$ at $15 \mathrm{~m}$ distance produces only $5.5 \mu \mathrm{W}$ due to a greater distance. On the other hand, RF-based wireless charging and information transfer at the same time is possible and it is known as simultaneous wireless information and power transfer (SWIPT) [52]. According to [53], two receiver architectures were considered in SWIPT networks, such as time switching-based relaying (TSR) protocol and power splitting-based relaying (PSR) protocol [54]. Particularly, an energy constrained node harvests energy from the RF signal that is broadcasted by the transmitter and it utilizes the collected harvested energy to forward the source information to the destination or another node. While the energy constrained node determines the required time that has to be shared for the energy harvesting circuit and the information processing circuit in TSR protocol, the time is evenly shared between the source-to-relay information transmission and the relay-to-destination information transmission, respectively, in PSR protocol. However, in PSR protocol, the energy constrained node on the first half of the block time determines the required power that has to be split into two separate circuits: EH and signal processing.

\subsubsection{Storage Capacity}

Characteristics of energy storage capacity play a significant role in implementing optimum transmission policy for EHNs. For instance, a large storage capacity can reduce the randomness effect of energy arrivals. In order to generalize this characteristic, the storage capacity types are sorted into two main modules; Harvest-Use-Save (HUS) module and Harvest-Use (HU) module.

HUS module: In an HUS module, the EH node has the ability to either use or save the converted electrical power based on network status. Outfitting an energy buffer with large capacity enhances the EHNs performance by minimizing the energy outage. Numerous 
papers have discussed the transmission policy for various types of EHNs using an HSU module $[12,16,17,55,56]$.

HU module: Mainly, transmission policy for EHN using an HU module is less complicated compared to an HUS module because EH nodes in an HU module are only required to immediately utilize the available harvested energy. Therefore, it is sufficient to design a transmission policy for a current time interval with no consideration of the whole transmission time. Recently, many papers have investigated the transmission policy for various types of EHNs using HU modules [57-59].

\subsection{Literature Review}

The lifetime of wireless networks can be prolonged via EH technology. Although supplying the low-cost devices with fixed energy supplies, such as batteries, and replacing the batteries when they are needed may achieve the goal of prolonging the wireless networks longevity, replacing batteries for such devices is either impossible or expensive, i.e., hazardous environments. Therefore, the EH technique is utilized to gather energy from the surrounding environment in order to overcome the bottleneck of fixed energy supplies [60]. Hence, this has grabbed wide interest in ambient energy-harvesting technologies.

EH techniques are divided into two types: conventional EH technique, which harvests energy from external energy sources that are not part of communication networks, such as those based on solar power, wind energy, etc. [60], and non-conventional EH technique, which replenishes energy from ambient Radio Frequency (RF) signals ( [52], [61], [62]). A problem of two-hop wireless EH and information processing communication system over AF relaying network was considered in [63]. Focusing on TS and PS receiver architecture, optimal TS factor $(\alpha)$ and PS factor $(\rho)$ that minimize the outage probability for an arbitrary relay are derived, respectively. Their analysis provides insights on how the system performance is affected by key parameters such as noise variances, source to relay distance, transmission 
rate, and energy conversion efficiency. The same communication system has been revisited in [64]. On the other hand, outage probability minimization problem based on relay selection in EH relay systems are recently attracted research interests. Particularly, this problem has been investigated for non-conventional EH network type [65]- [66]. Authors in [65] explore relay selection schemes based on the available CSI and largest available stored energy with the goal to minimize the outage probability for both causal and non-causal CSI cases. They conclude that although EH efficiency is an important factor for the outage performance, the tradeoff associated involving a number of relays in the system may outperform the limitation of EH efficiency.

Throughput maximization based on relay selection problem in EH relay systems has been investigated recently for non-conventional EH networks and conventional EH networks [62], [66], respectively. In [62], Aurhors proposed two relay selection schemes aiming to obtain the optimal tradeoff between energy transfer and ergodic capacity. In [66], offline and online joint relay selection, and source-relay transmit power allocation schemes are proposed that maximize the end to end system throughput over a finite number of transmission intervals. The optimal relay selection depends on both the CSI and the amount of harvested energy stored by the nodes.

Another body of work investigates the structure of optimal transnsmision policies for conventional EH technique in EH communication systems $[12,16,17,56]$. The transmission completion time minimization problem for a point-to-point transmission with EH constraints over Additive White Gaussian Noise (AWGN) channel has been studied in [16]. In addition, minimizing transmission completion time as well as maximizing throughput for a single-hop with EH constraints and over a fading channel have been investigated in [17] and [12]. Authors in [56] revisit the problems in $[12,17]$ and a novel approach: a Recursive Geometric Water-Filling (RGWF) algorithm, is proposed as an optimal transmission policy. Optimal power allocation for outage probability minimization in point-to-point fading channels with EH constraints has been studied in [67]. The system model was investigated for two cases: 
non-causal and causal energy state information, where they have been solved using offline and online power allocation algorithms, respectively. Although the system model was shown to be a non-convex problem, the innovative algorithm has solved the problem globally by exploiting some interesting properties of the outage probability function and one-dimension searching was only required. While, the previous-mentioned works concern resource allocation for EH point-to-point communications, in practice, it is not always possible for a source to communicate directly with the destination; for example, due to long distance or severe shadowing. Hence, deploying multi-hop and cooperative relays are significant in improving the coverage, throughput, and reliability of wireless networks. Transmission policy for a EH single-relay two-hop networks have been studied in [68-72]. In [68], throughput maximization problem for the two-hop communication system in the case of an EH source with two energy arrivals being solved using a cumulative curve algorithm. The optimal transmission policy with a non-EH source and an EH relay was developed in [69], while the optimal power allocation problem for the case where both the source and the relay are EH nodes with different delay constraints was solved in [70]. In addition, a power allocation strategy that maximizes the system throughput over a finite number of transmission time slots for EH two-hop networks under various delay constraints has been proposed in [71]. In [72], a Directional Water-Filling (DWF) algorithm, which is used to obtain optimum power allocation for a single-hop transmission, was adopted to gain an optimal transmission policy for a two-hop communication with EH constraints. Gunduz eviller $_{t} w o_{h} o p_{E} H$

\subsection{Contributions}

The main contributions of this thesis are the designs of transmission strategies for practical point-to-point and two-hop conventional EH communication systems, and they can be summarized as follows:

\# Resource Allocation for Two-Hop Communication with Energy Harvesting 


\section{Constraints Over Fading Channels:}

- Time scheduling and power allocation problem is investigated for a two-hop communication system with an EH source and a non-EH relay with objective of maximizing a short-term throughput with a given deadline.

- The low-complexity Recursive Geometric Water-Filling (RGWF) algorithm is modified and extended into a two-hop network scenario so as to optimize the offline resource allocation that maximizes the end-to-end system throughput for delay sensitive and delay tolerant relaying networks.

- For delay sensitive and delay tolerant cases, several online suboptimal low-complexity approaches are proposed that provide reasonable performance.

\section{\# Semi-Markov Decision Process (SMDP)-Based Resource Allocation for a Point-to-Point Communication System with Energy Harvesting Constraints:}

- This work concentrates on the online resource allocation problem for a point-to-point EH communication system, and a novel framework based on SMDP approach is formulated for the proposed system model with the objective of maximizing the network throughput.

- A SMDP-based policy is proposed that dynamically adapts to the changing of the channel status and the varying of arriving harvested energy and incoming data, and efficiently distributes the harvested energy.

- The objective of the framework is to obtain the optimal decision of resource allocation on the point-to-point EH communication system by maximizing the network throughput and reducing the service rejection probability.

\# SMDP-Based Online Cross-layer Adaptive Transmission Techniques for a Point-to-Point Communication System over Fading Channels: 
- A cross-layer adaptation transmission policy for an online point-to-point communication system with EH constraints over a Rayleigh fading channel based on SMDP under delay constraints is investigated.

- A novel framework based on the SMDP approach is formulated for the proposed system model with the objective of maximizing network throughput by optimally allocating the resource while maintaining minimum buffering delay and packet overflow.

- The proposed SMDP-based dynamic programming approach has been approved in being dynamically adaptive to the change of the channel and/or buffers states that optimally satisfy the BER requirements at the physical layer, and the overflow requirements at the data-link layer.

\subsection{Thesis Organization}

The remainder of the thesis is organized as follows:

Chapter 2: Resource Allocation and General Tools in EH Wireless Networks. Presents the resource allocation problems categorized and discussed according to the adopted approaches and network architecture. Then, basic elements, objectives and fundamental constraints of the EH wireless networks are well studied to provide the resource allocation design structure. Next, general tools for the resource allocation are explored and reviewed in the context of EH communication systems. Finally, a comprehensive overview of these categories and optimization strategies is provided.

Chapter 3: Resource Allocation for Two-Hop Communication with Energy Harvesting Constraints Over Fading Channels. Studies the traditional two-hop communication system for delay limited (DL) and delay tolerant (DT) relaying networks over fading channels, in which the source node transmits with power drawn from energy harvesting (EH) sources and the relay transmits with conventional non-EH sources. The throughput maximization problem for the proposed system model is addressed for DL and DT cases. 
It was found that the optimal power allocation algorithm for the single-hop communication system with EH constraints; namely, recursive geometric water-filling (RGWF), can be utilized as a guideline for the design of the two-hop system. First RGWF algorithm is introduced to show the advantages of the geometric approach in eliminating the complexity of the Karush-Kuhn-Tucker (KKT) condition as well as providing a closed-form and exact solutions to the proposed problem. Based on the RGWF algorithm, offline joint power allocation and transmission time scheduling schemes for DL relaying network and DT relaying network are proposed. as well as efficient online resource allocation schemes for both relays. The performance of the proposed schemes is evaluated via simulation and the results demonstrate that a network with delay tolerant ability provides better performance in terms of throughput.

\section{Chapter 4: SMDP-Based Resource Allocation for Wireless Networks with} Energy Harvesting Constraints. Considers a point-to-point communication system with energy harvesting constraints over a fading channel. A resource allocation framework based on a SMDP is proposed. The objective of the framework is to provide a solution for a throughput maximization problem in EH networks by maximizing the total long-term expected reward of the EH system. The system reward is derived by considering both the income and the cost of the EH wireless communications. Numerical results illustrated that

obtaining a transmission action policy based on the SMDP approach resulted in gaining higher throughput and provided guidelines for resource management in green communication with EH transmitters.

\section{Chapter 5: Cross-layer Adaptive Transmission Techniques for EH Systems} over Fading Channels. Investigates a cross-layer online adaptation transmission policy for a point-to-point communication system with EH constraints over a Rayleigh fading channel based on SMDP under delay constraints. Unlike most of the channel-dependent adaptation policies in the literature, the proposed policy chooses modulation constellation for EH networks dynamically depending on battery state, data buffer state in addition to channel state. 
Because the channel-dependent policy is physical layer optimized, it does not guarantee the upper-layer overflow requirement. Hence, a novel framework based on the SMDP approach is formulated for the EH wireless communication system with the objective of maximizing network throughput by optimally allocating the resource while maintaining minimum buffering delay and packet overflow. The proposed SMDP-based dynamic programming approach has been approved in dynamically adaptive the change of the channel and/or buffers states that optimally satisfy the BER requirements at the physical layer, and the overflow requirements at the data-link layer.

Chapter 6: Conclusion. Includes a summary of the research work, its outcomes, and conclusion. 


\section{Chapter 2}

\section{Resource Allocation Problems and General Tools}

\subsection{Wireless System Model}

In this thesis, we consider two main system model, namely a two-hop communication system with an EH source and a non-EH relay in Chapter 3, and a time-slotted single-input singleoutput (SISO) point-to-point communication system in which an energy harvesting source transmits latency-sensitive data over a fading channel in Chapter 4 and Chapter 5 . In both system model, the source is the energy harvesting node, where it does all the optimization part and the receiver is the base station, which is operated by a conventional power. We assume the source has always packets to transmit in Chapter 3, whereas data is arrived to the source with low arrival rate in Chapter 4 and high arrival rate in Chapter 5 . The major objective of this research is to investigate resource allocation schemes for practical point-to-point and two-hop conventional EH communication systems in order to support delay-tolerant and delay-sensitive applications with a more practical insight. 


\subsection{Resource Allocation Approaches}

In this thesis, two key approaches are utilized to solve the resource allocation problem for EH systems; namely, offline schemes and online schemes [12,17]. These approaches differ based on the available knowledge at the transmitter of random parameters that influence transmission, e.g., the channel SNR, the harvested energy, the number of incoming data bits. Each of these approaches has its merits and demerits. Therefore, depending on the problem's requirements and given parameters, one of the approaches can be adopted.

1) Offline: The offline approach is mainly based on the existence of noncausal information regarding the channel SNR, the harvested energy, the number of incoming data bits, etc., known a priori. In other words, the transmitter node has full knowledge (i.e., from the past, present, and future realizations) of these random parameters. It was shown that the study of offline transmission strategies have several advantages due to the following reasons. The optimal offline transmission strategy provides a bound on the achievable performance by any online strategy. For example, an upper bound can be obtained when the design objective is the maximization of some utility function. In opposition, a lower bound is obtained when the transmitter is designed to minimize a cost function. In addition, offline schemes can provide design insights that can help us to design an efficient online scheme.

2) Online: In the online approach, there is only causal information about the amount of harvested energy, the channel SNR, and the number of incoming data bits. The transmitter node has partial side information on these random parameters (including only past and present) and maybe some statistical information regarding its future behaviour. Therefore, in this case, online resource allocation schemes must be employed, taking into account the available information regarding the channel SNR, the harvested energy, and the incoming data bits. In general, an optimal online resource allocation scheme is formulated by computationally intensive stochastic dynamic programming (DP) [73]. However, in order to alleviate the computational cost of the optimal online scheme, suboptimal but lowcomplexity online schemes can be proposed. 


\subsection{Resource Allocation and Service Requirements}

In general, a transmission policy formulation problem mostly consists of three main components: objective function, a list of constraints, and a list of optimization variables, which are based on the applied optimization tools. The formulation of transmission policy for communication systems with EH constraints is more complicated compared to conventional communication systems because more constraints are required. Designing the transmission policy for non-EH networks is mainly based on the possibility of obtaining information of changeable parameters that affect transmission, such as Channel State Information (CSI) while designing the transmission policy for EH networks is based on the CSI as well as the amount of energy that is harvested and stored at the node's energy battery, which is random in nature. When the transmitter node has full information of the random parameters (including the past, present and future), the offline approach is considered whereas the online approach is applied when the transmitter node has partial side information of the channel condition and energy state (including only past and present).

On the other hand, throughput and delay are typically the most significant service requirements in the context of a point-to-point link [74]. Network throughput is defined as the total number of bits that are successfully transmitted through a channel in a certain time, and it testifies to the performance of the wireless channel. Delay considerations, are significant for the quickly increasing demands of real-time applications that require firm restrictions on packet delays. It must be remembered that the main objective of EH technology is to meet athe increasing energy demand of increasing numbers of wireless applications and devices, while lowering the emission of greenhouse gases to help achieve environmental sustainability. Moreover, supporting higher data rates under strict delay QoS requirements such as real-time applications increases the energy consumption. Therefore, it is not surprising that most researchers recently have focused on maximizing network throughput, through effective network control while taking into consideration the energy consumption and delay performance. 
There are several elements or objectives that could be targeted by the RA problem. These elements may be incorporated as the optimization principle objective or as a constraint that should hold.

\subsubsection{Objective Function}

There are several figures of merit that can be investigated when designing transmission strategies for EH communication systems. Some examples that have been considered in the literature are:

(1.) Maximization of the network throughput $[17,56,72,75]$.

(2.) Maximization of the energy efficiency [76].

(3.) Minimization of the total transmission time $[17,56]$.

(4.) Maximization of the outage probability [67].

This dissertation focuses on the maximization of the network throughput as a figure of merit, leaving the remaining ones as possible future research directions. In the dissertation, similary to [77], consider power allocation for effective capacity maximization for a point-topoint communication system over fading channel with $B \mathrm{~Hz}$. We assume ergodic stationary identically and independent distributed (i.i.d.) block-fading channel with fading duration $T$ (second) equal to the transmission epoch; channel power gain is constant during each epoch but independently from each others. Given Shannons formula, the corresponding instantaneous transmission rate (capacity) in epoch $i$ is given by:

$$
R\left[p_{i}\right]=\log \left[1+h_{i} \cdot p_{i}\right]
$$

where $h_{i}$ and $p_{i}$ denote the instantaneous (normalized) channel gain, and transmit power, respectively, in epoch $i=1,2, \cdots$. 


\subsubsection{Constraints}

Given any objective function, the system performance is usually limited by the available resources such as a finite amount of energy, bandwidth, transmission time, etc., or by some specific design requirements such as a minimum Signal to Noise Ratio (SNR) at the receiver, a maximum delay constraint, etc. In the following section, the common constraints that appear when designing transmission strategies for EH communication systems are summarized.

- Energy Causality Constraint: Energy causality constraint is uniquely considered in EH wireless communication. When the input power is subject to energy causality constraint, it means that energy must be available at the node battery and the total consumed energy cannot be more than the total available energy. In other words, the total consumed energy by the node at a certain instant in time must be no greater than the total energy that is harvested and stored in the battery at that time. An energy causality constraint can be stated as follows

$$
\sum_{i=1}^{l} \tau_{i} \cdot p_{i} \leq \sum_{i=1}^{l} E_{i}^{E H}, \quad \forall l
$$

where $\tau_{i}$ and $p_{i}$ are the transmission duration and the non-negative transmission powers of EH source, respectively, and $\sum_{i=1}^{l} E_{i}^{E H}$ is the cumulative harvested energy.

- Data Causality Constraint: This set of constraints applies when the data is dynamically received over time. Similar to the energy causality constraint above, the data causality constraint means that data can be transmitted if and only if data has been received and stored in the data buffer. Therefore, the total transmitted data by the node at a certain time instant must be no greater than the total arrival data on the data buffer at that time. Data causality can be expressed as:

$$
\sum_{i=1}^{l} \tau_{i} \cdot \log \left(1+h_{i} \cdot p_{i}\right) \leq \sum_{i=1}^{l} D_{i}, \quad \forall l,
$$


where $\sum_{i=1}^{l} D_{i}$ is the cumulative arrived data at the data buffer and $h_{i}$ is instantaneous (normalized) channel gain.

- Finite Energy Capacity Constraint: This constraint is for a finite battery case, where the communication device is limited in size and, hence, energy capacity is limited as well. Therefore, it is quite important for the proposed resource allocation to adapt the arrival of the harvested energy to ensure no energy packet is lost due to battery overflows. The adaptation mechanisms can be applied according to the EH-profile and the size of energy capacity such that energy expenditure is always less than the stored energy $E_{i}$ at any instant in time. Hence, the stored available energy must not exceed the size of energy storage capacity $E_{\max }$. The finite energy buffer causality constraint can be expressed as:

$$
\sum_{i=1}^{l} E_{i}^{E H}-\sum_{i=1}^{l} \tau_{i} \cdot p_{i} \leq E_{\max }, \quad \forall l,
$$

where $E_{\max }$ is the size of the energy capacity.

- Finite Data Buffer Constraint: Similar to the finite battery capacity constraint, the proposed transmission strategy must optimally adapt the data arrival rate and buffer size in order to ensure that no data packet is dropped due to buffer overflows. The finite data buffer causality constraint is described by:

$$
\sum_{i=1}^{l} D_{i}^{E H}-\sum_{i=1}^{l} \tau_{i} \cdot \log \left(1+h_{i} \cdot p_{i}\right) \leq D_{\max }, \quad \forall l,
$$

where $D_{\max }$ is the size of the node data buffer.

- Half-Duplex Constraint: This constraint indicates that the wireless communication devices cannot receive and transmit at the same time. Therefore, during source transmission, the relay or another node must be in silent mode and vice versa. Hence, 
transmission period must be shared between the nodes such that the optimum solution is achieved. The half-duplex causality constraint is given by:

$$
l_{i}^{s}+l_{i}^{r} \leq \tau_{i}, \quad \forall i,
$$

where $l_{1, i}$ and $l_{2, i}$ are the transmission durations for nodes (1) and (2), respectively, that are sharing the total time duration $\tau_{i}$ of the $i$ th epoch.

- QoS Constraint: QoS is a general term used for many user satisfaction related requirements. When a system is bounded with a certain performance, QoS constraints must ensure the system performance is within the limit, such as minimum SNR, maximum outage probability, maximum packet dropping rate, and maximum latency.

- Delay Constraint: Delay is an important metric in any wireless network especially for real-time applications such as voice and multimedia. By definition, delay refers to the amount of time that elapses until a packet is delivered to the destination from the moment that the message arrives at the data queue. Delay has two components; queueing delay: the amount of time a packet waits in a queue until it is executed, and transmission delay: the amount of time that the packet's bits take in the link. Both delay types need to be analyzed in order to characterize the delay performance.

\subsection{Optimization Framework and General Tools}

In this section, we explore and review the optimization strategies for throughput maximization problems in both conventional and EH communication systems. Depending on the objectives, these strategies can be categorized under several performance optimization criteria. In the following, a brief overview of these categories and optimization strategies is provided, and the characteristics of each are highlighted. 


\subsubsection{Single-hop Conventional Communication Systems}

Radio resources, such as power and bandwidth, must be well utilized and this can be classified as a Radio Resource Management (RRM) problem. Recently, various tools have been established for solving RRM problems. Water-Filling (WF) is a well-known information theory technique and it is a useful tool for RRM problems in wireless communication systems. Specifically, WF is widely utilized to determine power allocation strategies that maximize point-to-point channel capacity for offline settings by assigning more power to the channel with higher gains. As a result, the overall channel capacity is maximized.

\section{Problem and Algorithm}

In the following, the water-filling problem is introduced, which can be formulated as the following problem: given $P>0$, as the total power or volume of the water, find that

$$
\begin{array}{ll}
\max _{\left\{p_{i}\right\}_{i=1}^{K}} & \sum_{i=1}^{K} \frac{L_{i}}{2} \cdot \log \left(1+h_{i} p_{i}\right) \\
\text { subject to: } & 0 \leq p_{i}, \forall i, \\
& \sum_{i=1}^{K} p_{i}=P
\end{array}
$$

where $K$ is the total number of channels. Let $L_{i}, h_{i}$ and $p_{i}$ denote the time duration, the instantaneous (normalized) channel gain and the transmission power of the $i$ th channel, respectively. Since the constraints are such that (i) the allocated power must be nonnegative, and (ii) the sum of the power equals $P$, the problem (2.7) is called the water-filling (problem) with sum power constraint. On the other hand, the described channel principle can be extended to cover various communication systems' issues, i.e., a multi-carrier channel [78], a frequency-selective channel [79], a multiple input multiple output (MIMO) channel [80], etc. By interpreting the observed properties of the optimal power allocation scheme as a water-filling scheme, $p_{i}$ units of water are placed in a rectangular container with a bottom width of $\frac{L_{i}}{2},(\mathrm{i}=1, \ldots, \mathrm{K})$. To unify the parameter notation, through a change of variables, an equivalent target problem can be obtained as follows: 


$$
\begin{array}{ll}
\max _{\left\{p_{i}\right\}_{i=1}^{K}} & \sum_{i=1}^{K} \tau_{i} \cdot \log \left(1+h_{i} p_{i}\right) \\
\text { subject to: } & 0 \leq p_{i}, \forall i \\
& \sum_{i=1}^{K} p_{i}=P
\end{array}
$$

where $\tau_{i} \leftarrow \frac{L_{i}}{2}, h_{i} \leftarrow \frac{h_{i}}{L_{i}}$ and $p_{i} \leftarrow L_{i} p_{i}$. Note that the symbol $\leftarrow$ is the assignment operator.

In this section, two water-filling approaches are presented that solve the water-filling (problem) with sum power constraint. One is the conventional water-filling (CWF) method; another is the proposed geometric water-filling (GWF) method. The main principle of the WF approaches is to determine the optimum water level that maximizes the overall network throughput. Nevertheless, CWF and GWF algorithms are developed to solve this constrained optimization problem using different optimization approaches.

\section{Conventional Water-Filling (CWF)}

CWF is a wide WF algorithm umbrella approach that solves a power allocation problem with a sum power constraint under non-negative individual powers. Because of the non-linear equation and the inequality equation as shown in the problem (2.8), the resulting proposed system is non-linear. In the CWF algorithm, Karush-Kuhn-Tucker (KKT) conditions are utilized to find the solution to the proposed problem. KKT conditions can be considered, on the other hand, as a group of the optimal conditions. Below is the derived solution after applying the KKT conditions,

$$
\left\{\begin{array}{l}
p_{i}=\left(\mu-\frac{1}{h_{i}}\right)^{+}, \quad \forall i \\
\sum_{i=1}^{K} p_{i}=P, \\
\mu \geq 0 .
\end{array}\right.
$$

where $(z)^{+}=\max \{0, z\} . \mu$ is the water level chosen to satisfy the power sum constraint with equality $\sum_{i=1}^{K} p_{i}=P$. Eqn. (2.9) relates to the solution to the CWF problem (2.8). 


\section{Geometric Water-Filling (GWF)}

The GWF approach can be seen as a functional block that solves the same CWF RRM problem as in Eqn.(2.8). The GWF approach simplifies the CWF algorithm by eliminating the complexity of solving the non-linear system using KKT conditions, and takes a geometric approach that provides explicit solutions for and helpful insights into the problem and the solution. In the conventional water-filling algorithm, the optimum water level must be determined first, and then, the power allocation task is solved. In GWF, on the other hand, the highest channel under water $\left(i^{\prime}\right)$, is obtained instead, which is an integer. The water volume above the $i$ th channel $\left(P_{2}(i)\right)$ can be determined based on the height of the $i$ th fading channel $\left(d_{i}=\frac{1}{h_{i} \tau_{i}}\right)$ and the channel width $\left(\tau_{i}\right)$ as shown below,

$$
P_{2}(i)=\left[P-\sum_{k=l}^{K-1}\left(d_{i}-d_{k}\right) \tau_{k}\right]^{+}, \text {for } i=l, \ldots, K
$$

The main purpose of determining $P_{2}(i)$ is to find the maximal channel index $\left(i^{\prime}\right)$ that makes $P_{2}(i)$ positive. Hence, $i^{\prime}$ can be obtained using the following formula

$$
i^{\prime}=\max \left\{i \mid P_{2}(i)>0,1 \leq i \leq K\right\}
$$

Consequently, the allocated power of the $i$ th channel $(i=1,2, \ldots, K)$ is determined as follows:

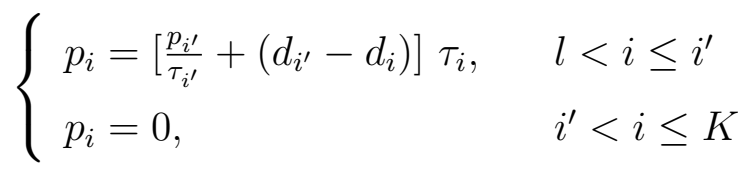

where the power level of the $i^{\prime}$ channel is obtained as follows:

$$
p_{i^{\prime}}=\frac{\tau_{i^{\prime}}}{\sum_{k=l}^{i^{\prime} \tau_{k}}} P_{2}\left(i^{\prime}\right)
$$

On the other hand, the GWF algorithm uses fewer computational resources compared 
to CWF. The worst-case computational complexity of the GWF approach has $(8 K+3)$ fundamental arithmetic, whereas the CWF approach has $O\left(K^{2}\right)$ fundamental arithmetic [81]. More detailed of GWF algorithm can be found in [81].

\subsubsection{Single-hop EH Communication Systems: Offline Setting}

EH communication is an advanced wireless communication technique that has attracted enormous research attention. Authors in [56] presented the RGWF algorithm that solves the optimum power allocation problem for a point-to-point data transmission with EH transmitter over a fading channel based on the GWF approach. Shannon capacity is adopted to achieve the maximum link capacity by determining the rate versus power relationship of the channel, given by:

$$
R\left[p_{i}\right]=\log \left[1+h_{i} p_{i}\right]
$$

where $h_{i}$ and $p_{i}$ are the instantaneous (normalized) channel gain and the transmission power at the $i$ th slot, respectively. The total transmitted bits for a given link in the $i$ th time slot with duration $\tau_{i}$, is given by:

$$
D(i, \tau)=\sum_{i} \tau_{i} \cdot R\left(p_{i}\right)
$$

\section{Problem and Algorithm}

In [56], throughput maximization problem for a single-hop system with EH constraints over fading channels is investigated. The non-negative EH node transmission power is defined as $p_{i}$ with non-negative transmission durations $\tau_{i}$, for $(i=1,2, \ldots, K)$, respectively. Moreover, $\sum_{i=1}^{l} E_{i}^{E H}$ is the cumulative harvested energy of the transmitter. When the input power is subject to energy causality constraint, it means that the energy harvested can only be used in the current time or in the future, but not in the past. In addition, the total consumed energy cannot be more than the total available energy, and an energy causality constraint is stated in Eqn.(2.2) 
In other words, by replacing the sum power constraint in Eqn.(2.8) with the EH causality constraint, the target problem can be defined as a water-filling problem with EH constraints. As a results, other approaches were utilized to solve this problem, and they are namely called directional water-filling (DWF) [17] and recursive geometric water-filling (RGWF) [56], which are extensions of CWF and GWF approaches, respectively. Hence, the throughput maximization problem can be formulated as follows,

$$
\begin{array}{ll}
\max _{\left\{p_{i}\right\}_{i=1}^{K}} & \sum_{i=1}^{K} \tau_{i} \cdot \log \left(1+h_{i} p_{i}\right) \\
\text { subject to: } & \sum_{i=1}^{l} \tau_{i} \cdot p_{i} \leq \sum_{i=1}^{l} E_{i}^{E H}, \quad \forall l, \\
& 0 \leq p_{i}, \quad 0 \leq \tau_{i}, \quad \forall i,
\end{array}
$$

Consequently, the objective function of this problem will be based on the total amount of data that the energy harvesting transmitter can forward to the destination over a given number of transmission time slots $K$.

\section{Directional Water-Filling (DWF)}

DWF was initially proposed in [17], which solved the presented problem (2.16) by adding EH constraints. Specifically, the authors in [17] consider the optimization of a point-to-point data transmission with an energy harvesting transmitter over a wireless fading channel. In the current work, the water-filling problem with $\mathrm{EH}$ constraints is introduced, which can be formulated into the following problem: given $E_{i}^{E H}>0$, as the amount of energy arrival at $i$ th epoch. Hence, the above problem has been solved using the DWF algorithm. The main feature of DWF is illustrated in Fig.(2.1), where $h_{i}$ is instantaneous (normalized) channel gain, i.e., low fading level and high fading level, which represent the good channel state and

bad channel state, respectively. Mainly, DWF aims to equalize the power as much as possible and it proceeds as follows: [81]

1. Fill each incoming energy until next energy arrival.

2. A water right-permeable material (the solid line that separates each three epochs) 


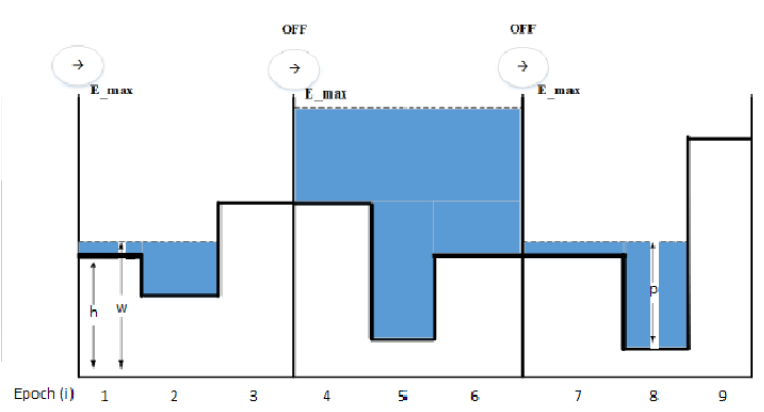

Figure 2.1: Fill the incoming energy until the next energy arrival

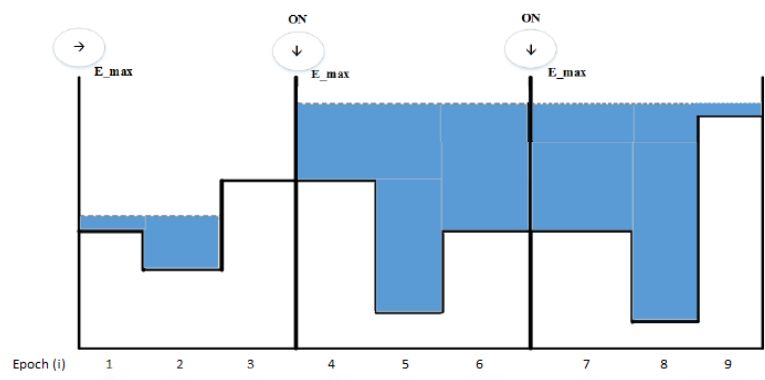

Figure 2.2: Equalizing the water level among the epochs

allows the available energy to be transferred to the right only, whereas energy transfer in the left direction is not allowed. (Because of energy causality).

3. Equalize the water level $w$ among intervals if the water level on the left-hand side is higher than the right side while keeping in mind the level of harvested energy cannot exceed $E_{\max }$. This is clearly shown in figure (2.2) at intervals $(4,5,6,7,8,9)$.

4. Eventually, the optimal power allocation is determined as the height of the water in each interval, i.e., $P_{i}^{*}=\left[w_{i}-h_{i}\right]^{+}$, where $w_{i}$ is the water level of the $i_{t h}$ epoch.

\section{Recursive Geometric Water-Filling (RGWF)}

Problem (2.8) has been solved based on the GWF scheme, which eliminates the complexity of employing the KKT multipliers. The RGWF algorithm, on the other hand, is a recursive version of the geometric water-filling algorithm that is utlized to solve problem (2.16) by 
obtaining optimum transmission power and maximizing the throughput of a single-hop EH communication system, while taking into account that channel conditions and harvested energy vary in time. In RGWF, GWF can be represented as a functional block that is recursively applied to sequentially solve the power allocation problem for energy harvesting transmission over fading channels. The exact optimal allocated power can be determined to provide insights into the given profile and the solution.

In the following, a description of the RGWF algorithm is presented.

1. Assuming $K$ is an integer that represents the total number of slots in a given transmission time (T), the assigned arrival harvested energy can be represented as $\left\{1, K,\left\{E_{i}^{E H}\right\}_{i=1}^{K}\right\}$.

2. RGWF sequentially processes the $(K)$ slots starting from the second time slot.

3. Assuming $(L)$ is the index of current processing slot, the power levels are updates for slot $(L)$ and its previous $(L-n)$ slots. Hence, a processing window is established.

4. GWF algorithm is applied to this window to obtain optimal power allocation for the slots that are assigned to this window by solving [(2.10)-(2.13)]. The common water level is then found.

5. The water level of the current process window is compared with the previous slot, and then, if the non-decreasing water level condition is satisfied, the allocated powers for the corresponding $(L)$ slots are obtained. Consequently, a new processing window will be created by moving to the next time slot. Otherwise, the current processing window will be extended with one slot in the left side and the same steps above are applied.

In this way, the completed optimum power allocation $\left\{p_{i}\right\}_{i=1}^{K}$ for the $i$ th time slot $(i=$ $1, \ldots, K)$ for a point-to-point $\mathrm{EH}$ communication system over fading channel are determined within finite loops. That is to say, RGWF can be written as a formal expression:

$$
\left\{p_{i}\right\}_{i=1}^{K}=R G W F\left(1, K,\left\{\tau_{i}\right\}_{i=1}^{K},\left\{h_{i}\right\}_{i=1}^{K},\left\{E_{i}^{E H}\right\}_{i=1}^{K}\right) .
$$


In the following, mapping is used as a first step to solve a two-hop communication system over fading channel with EH constraints.

\subsubsection{Single-hop EH Communication Systems: Online Setting}

The previous section presented an algorithm that provides optimum power allocation for a point-to-point EH communication system for the case where channel state information and energy arrival information are known in a non-causal manner. The presented algorithm will be utilized for maximizing the throughput of a two-hop wireless network with EH constraints. Since the aforementioned information is known only causally, the aim is to find the optimal transmission policy which maximizes the average total throughput of the network in an online manner. The optimal solution can be obtained using dynamic programming. Finding an optimal solution using dynamic programming is very complex because of the curse of dimensionality. To overcome this issue, discrete dynamic programming is used to cast the optimization problem as a semi-Markov decision process (SMDP). In the SMDP formulation, the set of transmission power levels is discrete and finite in contrast to general dynamic programming, where continuous power variations are allowed. Moreover, the channels are quantized to a finite number of states. The quantizations of channel and power level result in a state space of finite size and making the SMDP formulation mathematically tractable to solve the throughput optimization problem in the online setting. Introduced below are some of the basic concepts and terminology and discuss each ingredient of the SMDP problem.

\section{Semi-Markov Decision Process (SMDP)}

- Control Problem and Decision Epochs: In a stochastic control problem there are set of states, where in each state, some actions are executed that influence the environment; it may enter another state and action, repeatedly. In a stochastic system, a decision maker or a controller is in charge of controlling the behaviour of a certain stochastic system, which evolves over time. In MDP, this control is carried out by 
making decisions or taking actions at specific equal time instances. In other words, time is always discretized at equal time steps, where it may make a transition to another state. In this approach, decisions can be made only at fixed epochs $t=0,1, \ldots$. However, in many stochastic control problems the times between the decision epochs are not constant but random. A possible tool for analysing such problems is the semiMarkov decision model. In SMDP, these time steps do not have the same amount of time and, hence, some time steps might be longer than others.

On the other hand, the permanence in a certain state occurs for a random amount of the time, called the holding time $(\tau)$, following a given distribution. The holding time $(\tau)$ represents the time to the next transition. In other words, it indicates the time that the environment is going to spend in the current state before it transitions to a new state. Each state is associated with one or more reward variables called reward rates.

- States and Actions: Consider a dynamic system whose state is reviewed at random epochs. At those epochs, a decision has to be made and costs are incurred as a consequence of the decision made. The set of all such states is referred to as the state space $S$. Given that the present state of the system is $s \in S$, the scheduler can select an action $a$ from a set of allowable actions $A^{s}$ associated with this state. For each state $s \in S$, a set $\mathrm{A}(\mathrm{s})$ of possible actions is available. It is assumed that the state space $S$ and the action sets $\mathrm{A}(\mathrm{s}), s \in S$ are finite.

- Rewards and Transition Probabilities: Given that the controller takes an action $a \in A^{s}$ at the system state $s \in S$, the system will obtain a reward of $r(s, a)$. In other words, there will be an instant reward for every state and action pair. The function $r(s, a)$ is a real valued function defined by $S \times A$.

The future system state is affected by the action that is taken at the current state. Moreover, The system state in the next epoch is changed according to the transition 
probability. The transition probability; $p\left(s^{\prime} \mid s, a\right)$ can be defined as the probability that the system will be in state $s^{\prime} \in S$, given that the state of the system is $s$ and the action $a^{s}$ is selected.

\subsection{Chapter Summary}

This chapter presents the resource allocation problems that are categorized and discussed according to the adopted approaches and network architecture. Then, basic elements, objectives and fundamental constraints of the $\mathrm{EH}$ wireless networks are well studied to provide the resource allocation design structure. Finally, general tools for the resource allocation are explored and reviewed in the context of EH communication systems, and a comprehensive overview of these categories and optimization strategies is provided. 


\section{Chapter 3}

\section{Resource Allocation for Two-Hop}

\section{Communication with Energy}

\section{Harvesting Constraints Over Fading}

\section{Channels}

\section{$3.1 \quad$ Introduction}

Transmission polices for EH communication systems to maximize throughput have been well investigated recently. A throughput maximization problem for a single-hop with EH constraints over a fading channel has been investigated in [17] and [12]. Authors in [56] revisit the problem in $[12,17]$ and a novel approach: a Recursive Geometric Water-Filling (RGWF) algorithm, is proposed as an optimal transmission policy. However, as the harvested energy

Parts of Chapter 3 are presented at the IEEE International Conference on Wireless Communications Signal Processing (WCSP) in Nanjing, China [82], and published in the Journal Issue on Information and Communication Technology [83]. 
CHAPTER 3. RESOURCE ALLOCATION FOR TWO-HOP COMMUNICATION WITH ENERGY HARVESTING CONSTRAINTS OVER FADING CHANNELS

is mainly arrives randomly in a small amount, in single-hop EH networks, how to guarantee the satisfactory short-term performance is a challenging problem

Multi-hop transmission is used in order to expand the network size geographically using relays, thereby increasing the communication transmission range. Hence, it may utilize as a potential candidate to improve the short-term performance of EH communication networks. Two-hop communication systems with EH constraints have been studied recently, but only for some special cases. The authors in [69] propose an optimal transmission policy for the two-hop system model in the case of multiple EH packets arriving at the relay. In [68], a throughput maximization problem for the two-hop communication system in the case of an EH source with two energy arrivals has been solved using a cumulative curve algorithm. In [82], a Recursive Geometric Water-Filling (RGWF) algorithm, which is used to obtain optimum power allocation for a single-hop transmission, was adopted to gain an efficient transmission policy for a two-hop communication with EH Source (EH-S) and conventional Non-EH Relay (NEH-R) over a fading channel for a delay-limited network.

In this chapter, an efficient transmission policy of two-hop communication with energy harvesting constraints over fading channels for DS relaying network is investigated, as well as a DT relaying network under deterministic offline and online settings. By comparing our work with the existing results, the contributions of this work are summarized as follows:

- Novel solutions are presented to tackle a short-term throughput maximization problem with a given deadline of the two-hop communication system over a fading channel.

- The low-complexity RGWF algorithm is modified and extended into a two-hop network scenario so as to optimize the offline resource allocation that maximizes the end-to-end system throughput for DS and DT relaying networks.

- For DS and DT cases, several online low-complexity approaches are proposed that provide reasonable performance.

The remainder of this chapter is organized as follows. The system model and the adopted 
CHAPTER 3. RESOURCE ALLOCATION FOR TWO-HOP COMMUNICATION WITH ENERGY HARVESTING CONSTRAINTS OVER FADING CHANNELS

transmission policy are presented in Section 3.2. In Section 3.3, the throughput maximization problem is formulated and the developed algorithms for the DS case are proposed. The formulation of the throughput maximization problem and the proposed algorithms for the DT case are discussed in Section 3.4, followed by simulation results and chapter summary in Sections 3.5 and 3.6, respectively.

\subsection{System Model}

\subsubsection{Model Description}

In this section, the system model in [72] is adopted, which consists of a two-hop communication system with an EH-S and a half-duplex NEH-R over a fading channel, as shown in Fig. 3.1. The direct transmission between source and destination is negligible because of deep fading. For convenience, it is considered that the given total transmission time period is from $[0, T]$ including $\mathrm{K}$ epochs. Each epoch is represented as either new harvest energy arriving or change in the channel fading gain, or both. The time difference between epochs' instants $\left(t_{i-1}\right)$ th and $\left(t_{i}\right)$ th is called the $i$ th epoch, which is defined as $\tau_{i}=t_{i}-t_{i-1}$, for

$i=1,2, \ldots, K$. Moreover, $E_{i}$ is the corresponding amount of harvested energy and $\frac{1}{h_{i}}$ is the fading level in the $i$ th interval, for $i=1,2, \ldots, K$. Without loss of generality, it is assumed that $t_{0}=0$ and $t_{K}=T$. The terms epoch and interval are used interchangeably in this chapter. We assume the channel between the source-relay link $\left(h_{i}^{s}\right)$ experiences fading whereas $\left(h_{i}^{r}\right)$ is the AWGN channel on the relay-destination link for the $i$ th interval, $(i=1,2, \ldots, K)$. In addition, the available energy is utilized only for transmission purposes. Thus, Shannon capacity is adopted to achieve the maximum link capacity by determining the rate versus power relationship of the channel.

In this chapter, resource allocation schemes for a two-hop wireless communication with EH constraints over fading channels for delay-sensitive and delay-tolerant networks are proposed. In the following subsection, the advantage of a RGWF-EH profile in maximizing the 


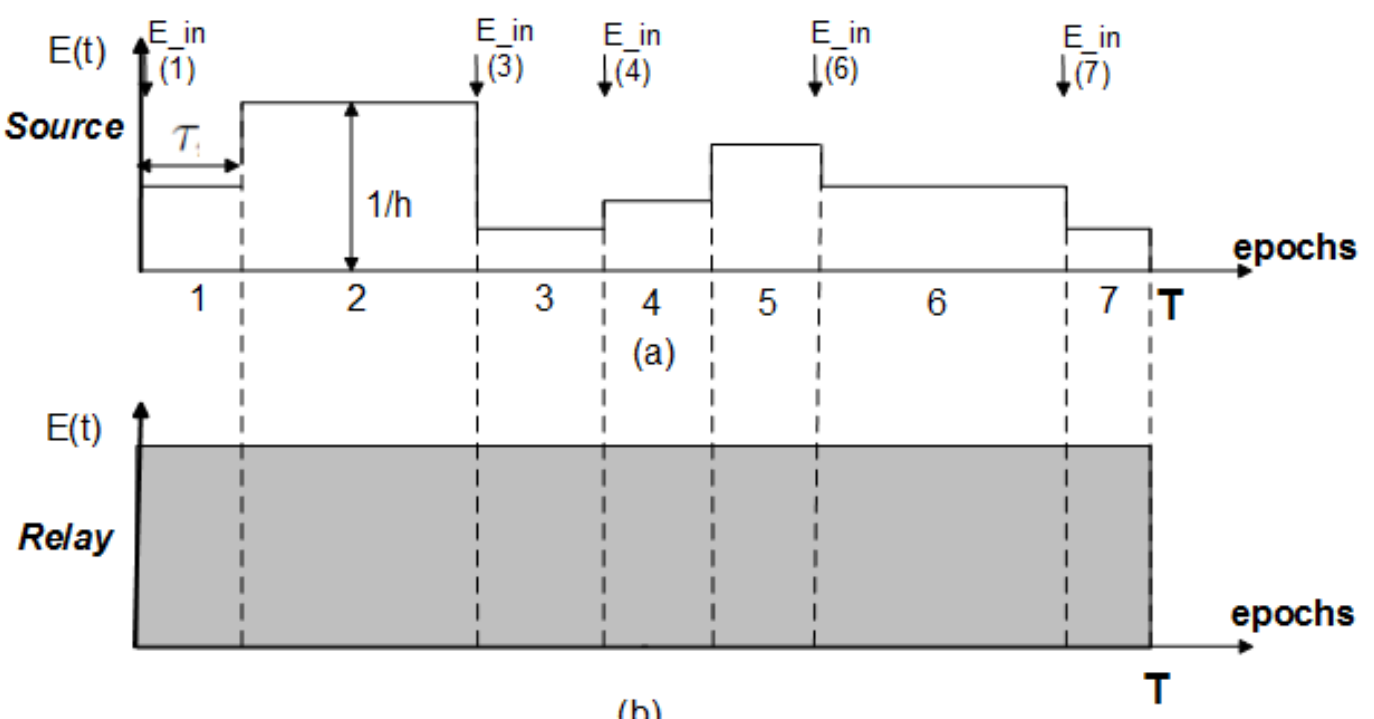

(b)

Figure 3.1: System model, $\mathrm{K}=7$ epochs in $[0, \mathrm{~T}]$ with (a) EH source, i.e., random energy arrivals $\left(E_{i n}\right)$, has a time-variant channel, and (b) non-EH relay, i.e., relay powered by battery, experiences an AWGN channel.

network throughput and the transmission policy for both delay networks' types is discussed.

\subsubsection{RGWF-EH Profile}

The RGWF algorithm has been shown to provide optimal power allocation for a single-hop over a fading channel [56]; as illustrated in Fig.(3.2-a), where the plain areas represent the fading gain and the shadowed areas represent the allocated power for the epochs. According to Fig.(3.2-a), it is easy to notice that more power is allocated to the epoch with less fading whereas less power is allocated to the high fading epoch, which leads to maximum throughput. Now, by only plotting the allocated power, the RGWF-EH profile is obtained as shown in Fig.(3.2-b). This profile can be used as a guideline for designing an efficient scheduling scheme that maximizes the network throughput for a two-hop communication system. On the other hand, a harvested energy arrival profile at the source node and a bit arrival profile at the destination are strongly related to each other. Since the source can transmit no data until energy is physically available, the EH profile at the source controls the number of the 
CHAPTER 3. RESOURCE ALLOCATION FOR TWO-HOP COMMUNICATION WITH ENERGY HARVESTING CONSTRAINTS OVER FADING CHANNELS

transmitted bits to the relay. Moreover, the bit arrival profile at the relay will be shaped based on the number of bits that have already been transmitted by the source. In addition, according to the energy causality constraints, the total power assigned up to the $i$ th epoch cannot be greater than the total harvested energy up to the same epoch. Consequently, EH profile forms the domain of the harvested energy usage, and all the feasible transmission polices will be narrowed to this domain.

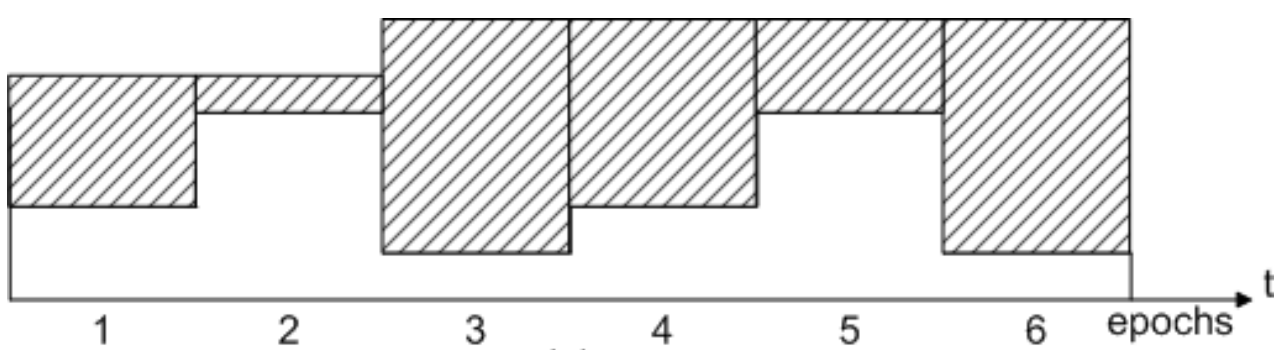

(a)

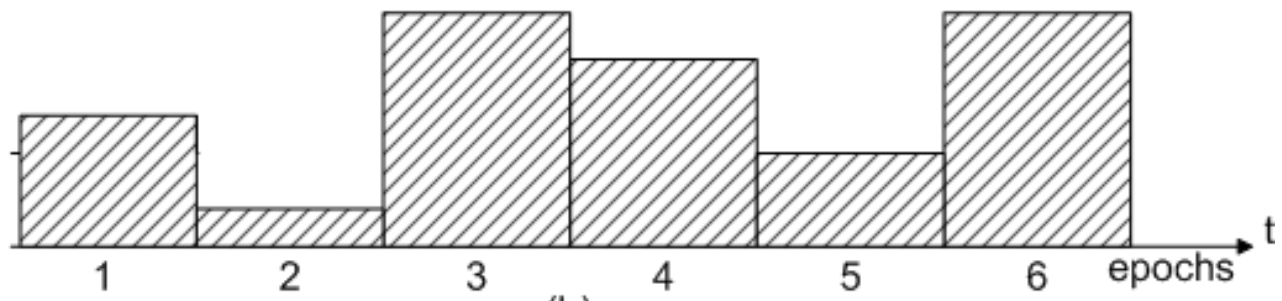

(b)

Figure 3.2: (a) Optimum power allocation for a single-hop with EH constraint over a fading channel using RGWF. (b) RGWF-EH profile.

\subsubsection{Transmission policy}

Earlier work [17] illustrates that the transmission rate/power of a node is constant between two sequential arrivals of energy. This policy is also kept in the current analysis. Although obtaining an optimal transmission policy for a single-hop transmission plays the core role of designing a two-hop communication system, the objective function for the two-hop transmission is dissimilar. Particularly, the objective function in single-hop transmission is to maximize the throughput in $(\mathrm{T})$, whereas the two-hop system has the same overview except 
CHAPTER 3. RESOURCE ALLOCATION FOR TWO-HOP COMMUNICATION WITH ENERGY HARVESTING CONSTRAINTS OVER FADING CHANNELS

the transmission must be through a relay node. This means that the transmission scheduled time must be divided between source-relay and relay-destination sessions. Therefore, to obtain an efficient transmission policy for two-hop transmission, source and relay power allocation as well as transmission scheduling need to be well planned in order to maximize the network throughput.

In addition, the transmission policy for the EH two-hop communication system is investigated based on both DT and DS cases. In a delay tolerant network, the source node keeps transmitting as long as it has a good channel condition, where it is defined as the channel fading gain is higher than the average threshold over all epochs, and has sufficient energy to transmit. The relay node, on the other hand, will start forwarding the received bits once the source pauses its transmission due to deteriorated channel conditions. In contrast, an example of non-delay tolerant networks is real-time applications that impose strict restrictions on packet delays such that the relay has to forward received packets as soon as they arrive, Whereas the data buffer at NEH-R is neglected in a delay-sensitive network, it is assumed that the data buffer in a delay tolerance network has infinite capacity at the relay node, and the EH-S has data all the time.

The following sections will show how to take advantage of the obtained RGWF-EH profile to maximize network throughput. The ordinary RGWF algorithm is only valid for a single-hop power allocation problem. In order to extend RGWF to two-hop transmission, a modified version of RGWF is proposed. For the throughput maximization problem with a source having an RGWF-EH profile, the transmission policy of a two-hop communication has the following properties:

1. The transmission rate/power of a node is constant within the $i$ th interval, for $i=$ $1,2, \ldots, K$
$p^{s}(t)=\left\{\begin{array}{ll}p_{i}^{s} & t \in L_{i}^{s} \\ 0 & t \in L_{i}^{r}\end{array}\right.$, 
CHAPTER 3. RESOURCE ALLOCATION FOR TWO-HOP COMMUNICATION

WITH ENERGY HARVESTING CONSTRAINTS OVER FADING CHANNELS

$p^{r}(t)=\left\{\begin{array}{cc}p_{i}^{r} & t \in L_{i}^{r} \\ 0 & t \in L_{i}^{s}\end{array}\right.$

where $L_{i}^{s}\left(L_{i}^{r}\right)$ is the set of the source (relay) transmission sets in the given time $[0, T]$.

2. Emptying both source energy and the relay data buffer at the end of the given transmission time $(T)$.

Based on the above discussed properties, it is concluded that a joint source and relay power allocation along with their scheduled time must be optimized such that a source transmits first and then it is followed by the relay at its allocated scheduling time. On the other hand, the total number of transmitted bits that are delivered from the source to the relay must be the same as the number of transmitted bits from the relay to the destination by a given time $(T)$.

\subsection{Throughput Maximization Problem for the DS case}

This section aims to maximize network throughput of a two-hop system with EH constraints for the DS network under offline and online knowledge of energy state information (ESI) and channel state information (CSI). EH-S and NEH-R are sharing the same epoch for the $i$ th interval $(i=1,2, \ldots, K)$ such that the $l_{i}^{s}+l_{i}^{r}=\tau_{i}$ constraint must be satisfied; where $\tau_{i}$ is the length of epoch $(i)$, whereas the total transmitted bits by both nodes must be equal for each $i$ th interval $(i=1,2, \ldots, K)$. The non-negative transmission powers of EH-S and NEH-R are defined as $p_{i}^{s}$ and $p_{i}^{r}$ with non-negative transmission durations $l_{i}^{s}$ and $l_{i}^{r}$, for $i=1,2, \ldots, \mathrm{K}$, 
CHAPTER 3. RESOURCE ALLOCATION FOR TWO-HOP COMMUNICATION WITH ENERGY HARVESTING CONSTRAINTS OVER FADING CHANNELS

respectively. The throughput maximization problem can be formulated as follows:

$$
\begin{array}{ll}
\max & \sum_{i=1}^{K} l_{i}^{r} \cdot \log \left(1+h_{i}^{r} p_{i}^{r}\right) \\
\text { subject to: } & \sum_{i=1}^{l} l_{i}^{s} \cdot p_{l}^{s} \leq \sum_{i=1}^{l} E_{i}^{E H}, \quad \forall l, \\
& l_{i}^{r} \cdot \log \left(1+h_{i}^{r} p_{i}^{r}\right) \leq l_{i}^{s} \cdot \log \left(1+h_{i}^{s} p_{i}^{s}\right), \quad \forall i, \\
& l_{i}^{s}+l_{i}^{r}=\tau_{i}, \quad \forall i, \\
& 0 \leq p_{i}^{s}, 0 \leq l_{i}^{s}, 0 \leq l_{i}^{r}, \quad \forall i,
\end{array}
$$

where $\sum_{j=1}^{i} E_{j-1}^{E H}$ is cumulative harvested energy at the source. Consequently, the objective function of this problem will be based on the total amount of data that NEH-R can transmit to the destination for the given total number of epochs $K$. The first condition is due to the energy causality constraints at the source, whereas the third constraint represents the half-duplex at the relay. Since small end-to-end delay is required, the equality of transmitted bits by both nodes at each time instant is shown in the following formula hence, the problem can be rewritten as following:

$$
\begin{array}{ll}
\max & \sum_{i=1}^{K} l_{i}^{r} \cdot \log \left(1+h_{i}^{r} p_{i}^{r}\right) \\
\text { subject to: } & \sum_{i=1}^{l}\left(\tau_{i}-l_{i}^{r}\right) \cdot p_{i}^{s} \leq \sum_{i=1}^{l} E_{i}^{E H}, \quad \forall l, \\
& l_{i}^{r} \cdot \log \left(1+h_{i}^{r} p_{i}^{r}\right)=\left(\tau_{i}-l_{i}^{r}\right) \cdot \log \left(1+h_{i}^{s} p_{i}^{s}\right), \quad \forall i, \\
& 0 \leq p_{i}^{s}, 0 \leq l_{i}^{r}, \quad \forall i,
\end{array}
$$

It is noted that the above property reduced the problem (3.2) into a convex optimization problem and it can be optimally solved using any conventional convex optimization technique [84]. In the following, optimal and suboptimal methods that solve the throughput maximization problem for a DS network will be discussed.

\subsubsection{Optimal Resource Allocation}

The most recent advanced method that provides the global optimal solution for both convex and non-convex optimization problems is the Interior-Point OPTimizer (IPOPT) algo- 
CHAPTER 3. RESOURCE ALLOCATION FOR TWO-HOP COMMUNICATION WITH ENERGY HARVESTING CONSTRAINTS OVER FADING CHANNELS

rithm [85]. Interior-point method provides optimal solutions for Non-Linear Programming (NLP) problems with enormous inequality constraints. The global convergence of the IPOPT method, which involves the primal-dual interior point algorithm, is proposed by Fletcher and Leyffer [86] utilizing a filter line search method. Specifically, the search direction is found by applying Newtons method into modified Karush-Kuhn-Tucker (KKT) equations. On the other hand, the filter term refers to a set of values that guarantee that the objective and constraint functions never return. A trial point is acceptable only if it achieves considerable progress towards the optimization goal and is not a member of the filter.

\subsubsection{Sub-optimal Resource Allocation}

In the transmission policy for the proposed problem without delay tolerance, there is a single source-relay stage pair in each epoch. Particularly, source and relay stages for the $i$ th RGWF interval $(i=1,2, \ldots, K)$ are defined as $l_{i}^{s}=\left[t_{i-1}, t_{i-1}+t_{i}^{\prime}\right), l_{i}^{r}=\left[t_{i-1}+t_{i}^{\prime}, t_{i}\right)$ respectively, where the time instants, $t_{i-1}, t_{i}$, and $t_{i}^{\prime}$, are the beginning, the ending of the $i$ th epoch, and the time when transmission is switched to the relay, respectively. The transmitting powers of the $i$ th RGWF interval $(i=1,2, \ldots, K)$ for the EH-S and NEH-R are $p_{i}^{s}=P_{i}^{E H}, p_{i}^{r}=P_{M}^{R}$ respectively, where $P_{i}^{E H}$ is the allocated power that is obtained based on applying the RGWF algorithm considering only the source-relay link, and $P_{M}^{R}$ is the relay power, which is given and fixed. Consequently, by applying Eqn.(3.3), the initial scheduled time is obtained for the $i$ th interval $(i=1,2, \ldots, K)$

$$
t_{i}^{\prime}=\frac{\tau_{i} \cdot \log \left(1+P_{M}^{R}\right)}{\log \left(1+P_{M}^{R}\right)+\log \left(1+h_{s r, i} P_{i}^{E H}\right)} .
$$

In the case of an ordinary RGWF, where only a single-hop is involved in the transmission, the power is allocated to the whole $i$ th interval, which includes source and relay transmission period. In this system model, the relay shares the interval with the source and therefore, the RGWF algorithm must be modified in order to guarantee that the power allocation 
CHAPTER 3. RESOURCE ALLOCATION FOR TWO-HOP COMMUNICATION

WITH ENERGY HARVESTING CONSTRAINTS OVER FADING CHANNELS

algorithm is exactly applied to the length of source stage. Otherwise, the allocated power on the relay stage, which is a result of applying RGWF to the whole epoch $\left(\tau_{i}\right)$, will be considered as wasted power. To overcome the potential problem of assigning source power to the relay transmission period, the source transmit power is re-allocated for the $i$ th epoch $(i=1,2, \ldots, K)$ as follows:

$$
P_{i}^{*}=P_{i}^{E H} \cdot \frac{\tau_{i}}{t_{i}^{\prime}} .
$$

The optimum time scheduling will be re-determined based on the optimum source transmitting power $\left(P_{i}^{*}\right)$, and subsequently, on the initial scheduled time $t_{i}^{\prime}$. Hence, the optimum scheduled time for the $i$ th epoch $(i=1,2, \ldots, K)$, is determined as follows:

$$
t_{i}^{*}=\frac{\tau_{i} \cdot \log \left(1+P_{M}^{R}\right)}{\log \left(1+P_{M}^{R}\right)+\log \left(1+h_{s r, i} P_{i}^{*}\right)},
$$

It can be seen that the solution given in (3.5) is unique and satisfies the two-hop transmission policies that are discussed in the beginning of this section. The proposed scheme is refereed to as the Relay In Partial Epoch (RIPE) algorithm based on RGWF.

\subsubsection{Pre-defined Data Rates (PDDR) Based}

This subsection discusses suboptimal performance considering the same problem set-up with only causal knowledge of CSI and ESI. The pre-defined multi-rate wireless technology is adapted in order to support the two-hop DS network with EH constraints. The rate adaptation techniques involve scheduling algorithms based on instantaneous information of source $\operatorname{SNR}\left(\gamma_{i}^{s}\right)$. It is assumed that the fixed relay $\operatorname{SNR}\left(\gamma^{r}\right)$ is the only parameter that must be known in advance. Since the relay node is powered by a fixed energy source, relay SNR is assumed to be higher than any instantaneous SNR of source node. Hence, resource allocation is obtained based on $\left(\gamma_{i}^{s}\right)$ only, where it is computed as follows:

$$
\gamma_{i}^{s}=h_{i}^{s} \cdot \frac{2 E_{i}^{E H}}{\tau_{i}}
$$


CHAPTER 3. RESOURCE ALLOCATION FOR TWO-HOP COMMUNICATION

WITH ENERGY HARVESTING CONSTRAINTS OVER FADING CHANNELS

The algorithm is proposed as follows. The maximum number of bits that the link can afford $\left(B_{\max }\right)$ is determined as follows:

$$
B_{\max (n)}=\frac{\tau_{i}}{2} \cdot \log \left(1+\left(\gamma^{r}\right)\right)
$$

Then, $(L)$ pre-defined multi-rate is computed by dividing $\left(B_{\max (n)}\right)$ into $\left(\frac{1}{L}\right)$ scale factors for $(\mathrm{n}=1, \ldots, \mathrm{L})$ where $\left(B_{\max (1)}\right)$ is the highest possible transmitted bits and $\left(B_{\max (L)}\right)$ is the lowest possible transmitted bits that the source can transmit in its scheduled time. On the other hand, the corresponding relay scheduled time that the relay needs to re-transmit $\left(B_{\max (n)}\right)$ is determined as follows:

$$
t_{\max (n)}^{r}=\frac{B_{\max (n)}}{\operatorname{rate}\left(\gamma^{r}\right)}
$$

Finally, the source scheduled time for $i$ th epoch $\left(t_{i}^{s}\right)=\frac{B_{\max (n)}}{\operatorname{rate}\left(\gamma_{i}^{s}\right)}$ is computed. If $t_{i}^{s} \leq\left(\tau_{i}-\right.$ $t_{\max (n)}^{r}$ ), then, $B_{\max (n)}$ is the maximum number of bits that can be transmitted for epoch $(i)$. Otherwise, $(n)$ increases by one until $t_{i}^{s} \leq\left(\tau_{i}-t_{\max (n)}^{r}\right)$ condition is satisfied. The above steps are applied for the $i$ th interval $(i=1,2, \ldots, K)$. The following pseudo-code summarizes our algorithm.

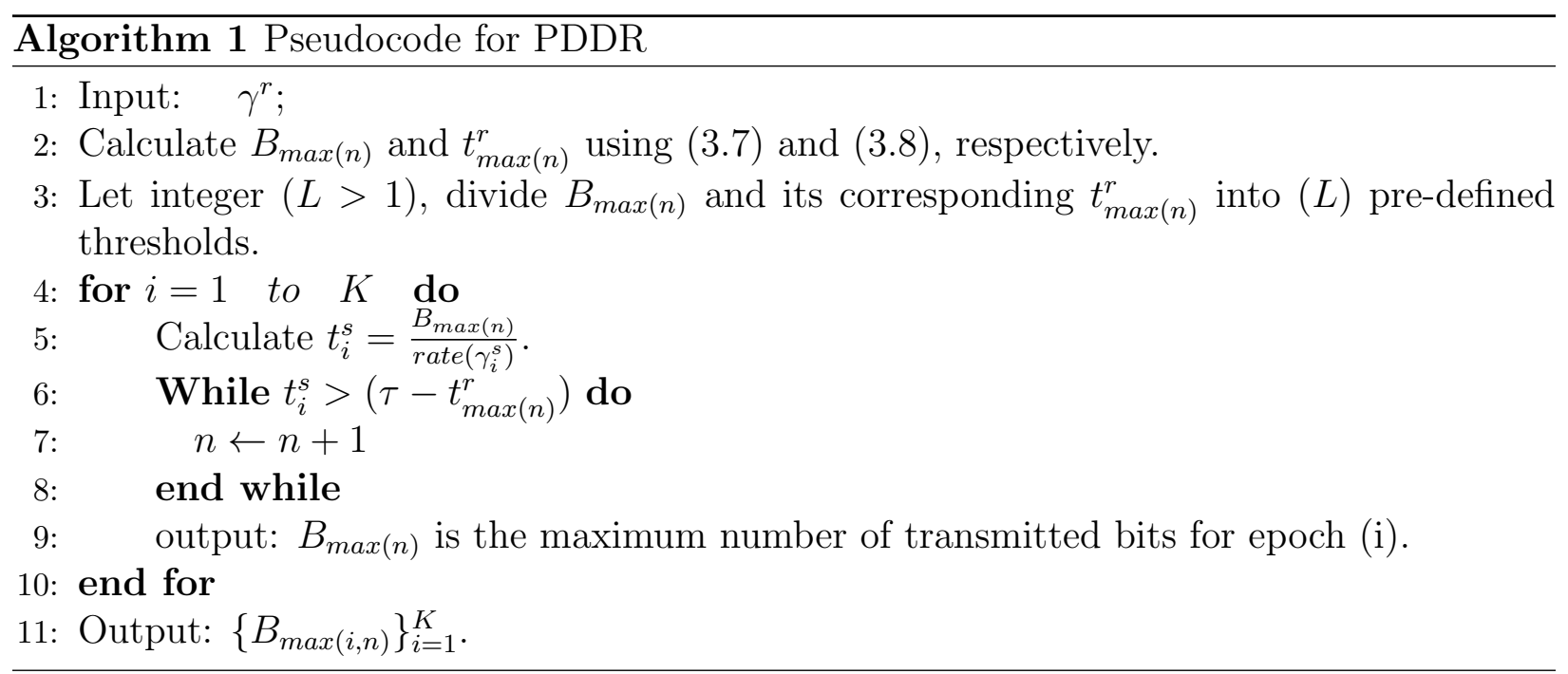


CHAPTER 3. RESOURCE ALLOCATION FOR TWO-HOP COMMUNICATION WITH ENERGY HARVESTING CONSTRAINTS OVER FADING CHANNELS

\subsection{Throughput Maximization Problem for the DT Case}

In this section, maximizing the number of bits delivered to the two-hop system with EH constraints is considered using a deadline $T$ for the DT network. A delay tolerance network is desirable when the network has no constraint on the end-to-end delay. Unlike the delaysensitive network, the epoch is never being shared between nodes; however, it is either being allocated to the EH-S or NEH-R for the $i$ th interval $(i=1,2, \ldots, K)$ such that the total transmitted bits by both nodes must be identical by the end of the $K$ time slots. The throughput maximization problem for the delay tolerance network can be formulated as follows:

$$
\begin{array}{ll}
\max & \sum_{i=1}^{K} \delta_{i} \cdot \tau_{i} \cdot \log \left(1+h_{i}^{r} p_{i}^{r}\right) \\
\text { subject to: } & \sum_{i=1}^{l}\left(1-\delta_{i}\right) \cdot \tau_{i} \cdot p_{i}^{s} \leq \sum_{i=1}^{l} E_{i}^{E H}, \forall l, \\
& \sum_{i=1}^{K-1} \delta_{i} \cdot \tau_{i} \cdot \log \left(1+h_{i}^{r} p_{i}^{r}\right) \\
& \leq \sum_{i=1}^{K-1}\left(1-\delta_{i}\right) \cdot \tau_{i} \cdot \log \left(1+h_{i}^{s} p_{i}^{s}\right), \\
& \sum_{i=K-1}^{K} \delta_{i} \cdot \tau_{i} \cdot \log \left(1+h_{i}^{r} p_{i}^{r}\right) \\
& =\sum_{i=K-1}^{K}\left(1-\delta_{i}\right) \cdot \tau_{i} \cdot \log \left(1+h_{i}^{s} p_{i}^{s}\right), \\
& \delta_{i} \cdot\left(1-\delta_{i}\right)=0, \forall i, \\
& p_{i}^{s} \geq 0, p_{i}^{r} \geq 0, \tau_{i} \geq 0, \delta_{i} \in\{0,1\} \forall i,
\end{array}
$$

where $\sum_{j=1}^{i} E_{s, j-1}^{E H}$ is the cumulative harvested energy values for the source. According to the second constraint, which shows the data causality, the amount of data transmitted by EH-S is higher than or equal to the data transmitted by the relay at any instant in time. Consequently, the objective function of this problem will be based on the total amount of data that NEH-R can transmit to the destination for the given total number of epochs $K$. The above problem is a convex Mixed Integer Non-Linear Program (MINLP) since the integer variable $\delta_{i}$ is either $(1)$ or (0); i.e., $\delta_{i} \in\{0,1\}$ and many advanced algorithms recently have been proposed to solve this type of problem efficiently. In this chapter, several schemes are proposed that solve the proposed problem in offline and online settings. 
CHAPTER 3. RESOURCE ALLOCATION FOR TWO-HOP COMMUNICATION WITH ENERGY HARVESTING CONSTRAINTS OVER FADING CHANNELS

\subsubsection{Optimal Resource Allocation}

The IPOPT algorithm is the most recent advanced method that provides the global optimal solution for NLP optimization problems, and the Advanced Process Optimizer (APOPT) algorithm is approved to solve many large-scale optimization problems, such as NLP, MILP and MINLP problems. APOPT is an open source software solver that provides global optimal solutions for convex MINLP problems [87].

On the other hand, the problem in (3.9) can be simply solved using the exhaustive search algorithm by giving $\delta_{i}$, for the $i$ th interval $(i=1,2, \ldots, K)$. Hence, the only variables that need to be optimized are $\left(p_{i}^{s}, p_{i}^{r}\right)$ for all possible combinations of $\delta_{i}$, for the $i$ th interval $(i=1,2, \ldots, K)$, and only a $\delta_{i}$ 's combination that maximizes the overall throughput will be chosen. Because of the complexity of the exhaustive search algorithm especially for large numbers of time slots, the exhaustive search approach will not be applied in this paper.

\subsubsection{Sub-Optimal Resource Allocation}

To solve the problem (3.9) heuristically, the whole epoch will be either allocated to the source node or the relay node based on $\left(\gamma_{i}^{s}\right)$ for the $i$ th interval $(i=1,2, \ldots, K)$. First, the RGWF approach will be applied to the source-relay link for the $i$ th RGWF interval $(i=1,2, \ldots, K)$. Hence, the transmitting powers of the $i$ th RGWF interval $(i=1,2, \ldots, K)$ for the EH-S

and NEH-R are $p_{i}^{s}=P_{i}^{E H}, p_{i}^{r}=P_{M}^{R}$ respectively. The threshold average capacity $\left(\theta_{\bar{c}}\right)$ for $(K \rightarrow \infty)$ can be formulated as

$$
\theta_{\bar{c}}=\frac{\sum_{i=1}^{K} \gamma_{i}^{s}}{K}=\frac{\sum_{i=1}^{K} h_{i}^{s} p_{i}^{E H}}{K}
$$

The whole interval is assigned to the source set $\left(L_{i}^{s}\right)$ if $\left(\gamma_{i}^{s} \geq \theta_{\bar{c}}\right)$ or it is allocated to the relay set $\left(L_{i}^{r}\right)$, otherwise. Moreover, the first interval is allocated to the source set, whereas the last epoch is allocated to the relay. Eventually, the RGWF algorithm is utilized again and it will be only applied to the intervals that are assigned to the source node $\left(\tau_{i}^{s}\right)$. To 
CHAPTER 3. RESOURCE ALLOCATION FOR TWO-HOP COMMUNICATION

WITH ENERGY HARVESTING CONSTRAINTS OVER FADING CHANNELS

avoid a vital problem that results in $p_{i}^{r} \neq 0$ even when the relay node does not have data to send, the proposed algorithm solves that potential problem as it is shown in algorithm. (2). The "for" loop (line 15 to 23) ensures that the bit arrival profile at the relay will be shaped based on the number of bits that have been transmitted by the source and the relay node only transmits if it has stored data in the buffer. The proposed algorithm is referred to as Relay In Demand using Average Capacity (RID-AC). Algorithm 2 shows the pseudo-code for this scheme.

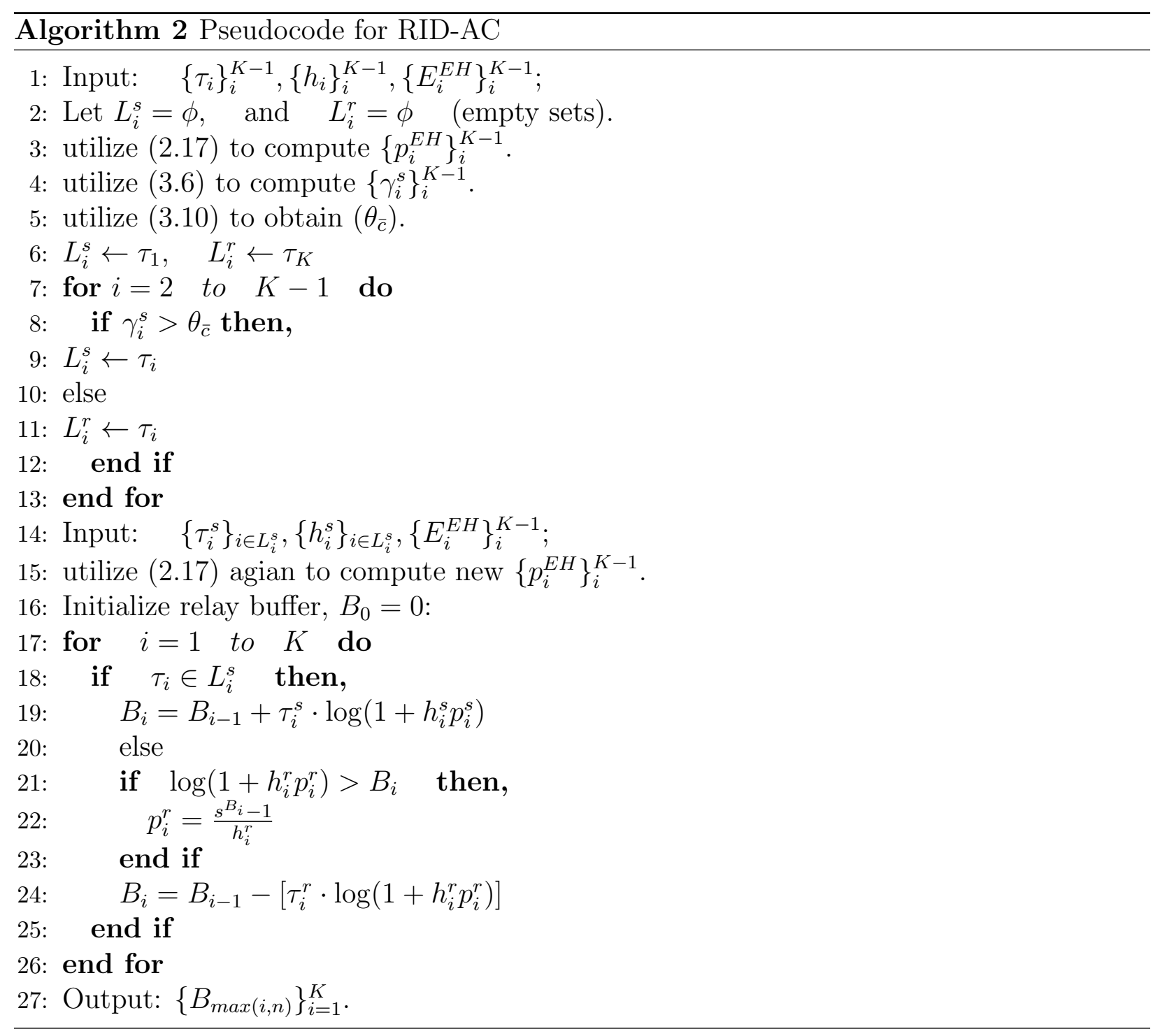


CHAPTER 3. RESOURCE ALLOCATION FOR TWO-HOP COMMUNICATION WITH ENERGY HARVESTING CONSTRAINTS OVER FADING CHANNELS

\subsubsection{Relay In Demand using Average Fading (RID-AF) Based}

It is well-known that only causal information of channel status and harvested energy is available for real scenarios of resource allocation in wireless communication. This is to say, an efficient low-complexity scheme is proposed that solves (3.9) with knowledge of CSI only. Similar to the sub-optimal performance of offline transmission policy using average capacity as a threshold, the whole epoch will be either allocated to source node or relay node. However, since only CSI is known in advance, the epoch allocation is determined based on the channel fading gain $\left(h_{i}^{s}\right)$ only as a threshold. Specifically, the epoch is allocated to source or relay for the $i$ th interval $(i=1,2, \ldots, K)$ as

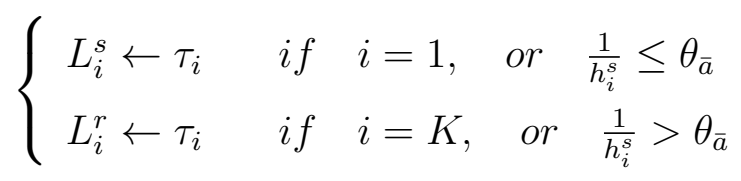

where the threshold based on the average fading $\left(\theta_{\bar{a}}\right)=\kappa \cdot \frac{\sum_{i=1}^{K} \frac{1}{h_{i}}}{K}$, and $(\kappa)$ is a scalar factor. The epoch $\left(\tau_{i}\right)$ is considered to be dedicated to the source in two cases: first at the beginning of transmission; second, when the channel fading gain is higher than the threshold. In the second case, the $i$ th epoch is dedicated to the relay transmission. Although the proposed solution is heuristic, it has been shown in the numerical results that the proposed algorithms in a delay-tolerant network achieved higher throughput compared to the proposed algorithms in a delay-sensitive network as well as Algorithm 2 in [72] with the price of no-bounded delay. The proposed algorithm is referred to as Relay In Demand using Average Fading (RID-AF). The following flow chart demonstrates the RID-AF algorithm, as it is illustrated in Fig.3.3.

\subsection{Simulation Results}

In this section, simulation results are provided to evaluate the performance of the proposed offline and online resource allocation algorithms for both delay-tolerant and delay-sensitive 


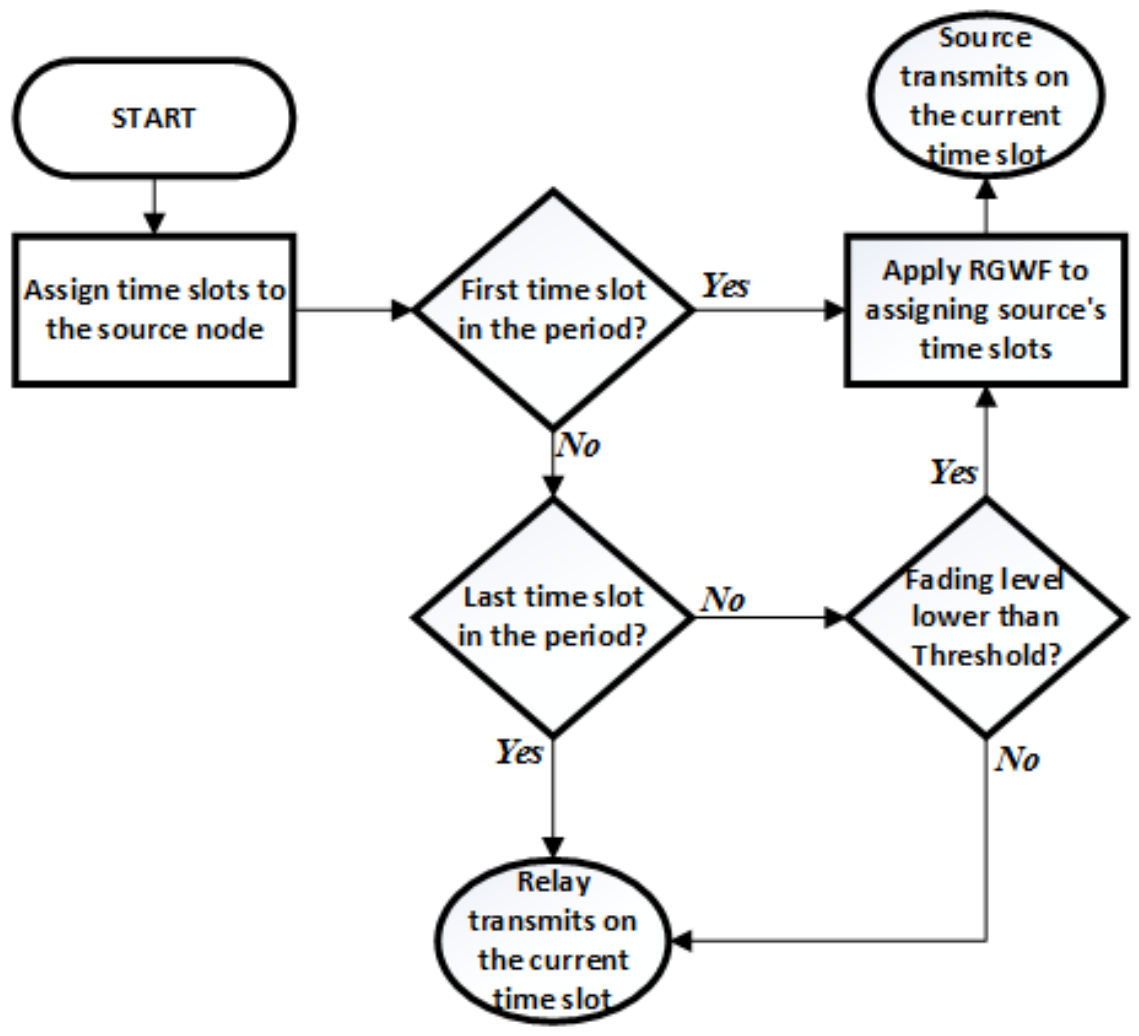

Figure 3.3: Flow chart of RID-AF algorithm. 
CHAPTER 3. RESOURCE ALLOCATION FOR TWO-HOP COMMUNICATION WITH ENERGY HARVESTING CONSTRAINTS OVER FADING CHANNELS

networks. We consider a band-limited fading channel in the source-relay link and additive white Gaussian noise channel in the relay-destination link, with bandwidth $(B W)=1 \mathrm{MHz}$ and noise power spectral density $N_{0}=10^{-19} \mathrm{~W} / \mathrm{Hz}$. The path loss and the transmission block length are assumed to be $100 \mathrm{~dB}$ and $100 \mathrm{~ms}$, respectively. Consequently, the rate

power function is determined as $r=\log \left(1+\frac{h \cdot P}{10^{-3}}\right)$. The method uses i.i.d. Rayleigh fading channels, where the gain $h$ follows Rayleigh distribution with mean $(\bar{m}=5)$ and $h=1$ for AWGN channels. The Energy Harvesting rate $P_{i}^{E H}$, on the other hand, follows a Poisson distribution with arrival rate $(\lambda=1)$, which is multiplied in the unit of the average EH rate for the source $\left(E_{o}^{E H}=[1,2, \ldots, 10]\right)$. The simulation was done using Matlab and it was run for 10000 random EH realizations.

\subsubsection{Performance of RGWF algorithm for a point-to-point com- munication}

Fig.3.4 shows the average throughput versus the source average EH rate with infinite energy capacity over a Rayleigh fading channel. The RGWF scheme performance is compared with a conventional non-EH source, where the same total amount of energy is available to allocate at the start of the process; and the baseline performance, where the source node starts its transmission as soon as the harvested energy arrives over a Rayleigh fading channel. It is shown that with the increasing rate of the harvested energy, the throughput loss of the RGWF over the non-EH network is more significant due to the fact that a higher amount of harvested energy has to follow the causality constraint, and therefore, loss flexibility of allocation. On the other hand, the proposed RGWF outperforms the baseline scheme in the entire range of the energy arrival rate. 
CHAPTER 3. RESOURCE ALLOCATION FOR TWO-HOP COMMUNICATION

WITH ENERGY HARVESTING CONSTRAINTS OVER FADING CHANNELS

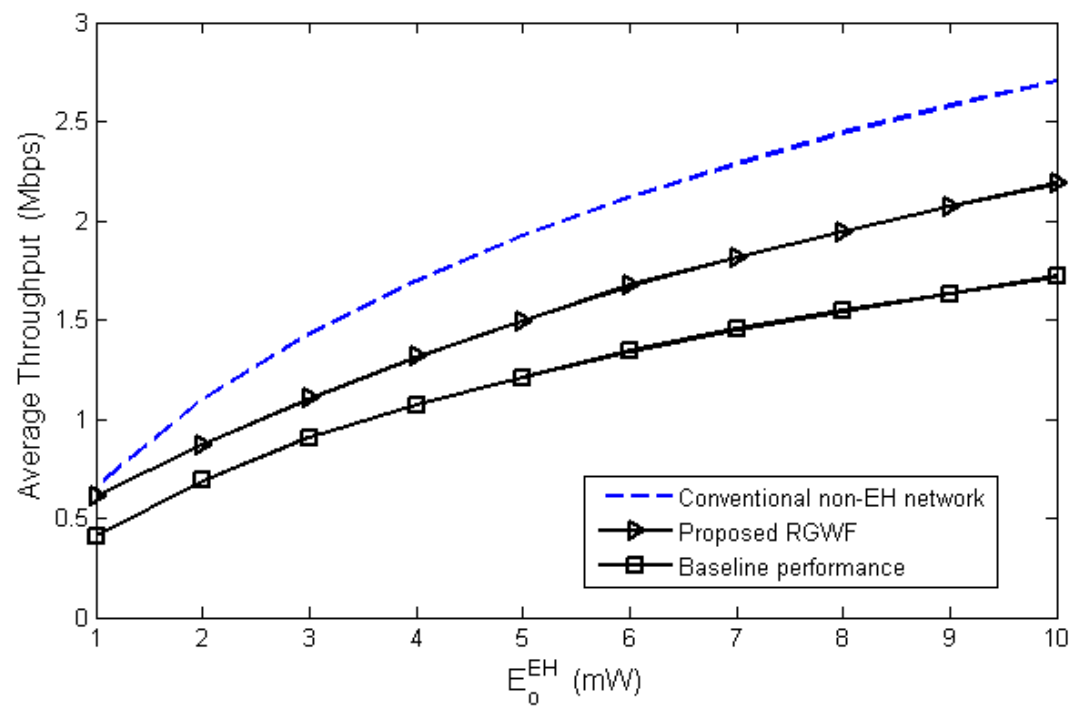

Figure 3.4: Simulation results of the throughput versus the source EH rate for a single-hop over a Rayleigh channel with $\bar{m}=5$.

\subsubsection{Resource Allocation Schemes for Delay-Sensitive Two-hop Communication}

In this subsection, we show the performance of the proposed resource allocation schemes for delay-sensitive relaying in a two-hop wireless network with an EH source over a Rayleigh fading channel, whereas the non-EH relay link experiences an AWGN environment. The throughput performance of the proposed algorithms to the baseline algorithms are coompared where fixed power allocation and time scheduling are applied to both nodes. For example, each epoch is divided into two-equal time slots and they are assigned to the source and the relay respectively. All proposed algorithms are also compared with the upper bound of the short-term throughput for conventional non-EH nodes.

Fig.3.5 shows the impact of high channel SNR and low channel SNR versus the throughput when the channel link faces Rayleigh fading. The figure shows the average throughput for the resource allocation schemes proposed for a delay-sensitive network versus $\left(E_{o}^{E H}\right)$ for $(K \rightarrow \infty)$ and with a constant relay peak transmitting power $P_{M}^{R}=10 \mathrm{~mW}$. It is notice- 
CHAPTER 3. RESOURCE ALLOCATION FOR TWO-HOP COMMUNICATION WITH ENERGY HARVESTING CONSTRAINTS OVER FADING CHANNELS

able that the average throughput increases as $E_{o}^{E H}$ increases for all considered schemes. As expected, it can also be observed that the performance of the offline proposed resource allocation scheme is superior to the online schemes for all $\left(E_{o}^{E H}\right)$. The reason behind this is the fact that the non-causal information of CSI and harvested energy is available before starting the transmission in offline schemes whereas only causal information of CSI and harvested energy is available for online case.

Fig.3.5 can be analyzed from two perspectives. First is the large throughput gap between the proposed RGWF-based algorithm (RIPE) and the baseline policy; second, it reaches the upper bound policy. In addition, when the network is delay-sensitive, it is clear that the proposed RIPE algorithms based on data rates alternate exactly between the upper bound and baseline performance, which indicates that the throughput performance is degraded in terms of less complexity and less feedback overhead. In addition, the proposed online RIPE scheme with 10-rates performs better than the proposed online RIPE scheme with 5-rates when source link faces the Rayleigh channel due to the fact that more data rates are available as options, especially when the SNRs vary among the epochs. Moreover, it is observed that the difference between the performance of PDDR-10 and PDDR-5 rates increases with increasing $E_{o}^{E H}$.

\subsubsection{Resource Allocation Schemes for Delay Tolerant Two-hop Communication}

This subsection discusses the performance of the proposed resource allocation schemes for delay tolerant relaying in a two-hop wireless network with EH source over a Rayleigh fading channel, whereas the non-EH relay link has an AWGN channel. The throughput performance of the proposed offline and online algorithms are compared to the baseline scheme where fixed power allocation and slot allocation are applied to both nodes. For example, assuming the total number of epochs are even, the odd epochs are assigned to the source and even epochs are assigned to the relay. All the proposed algorithms are also compared with the upper 
CHAPTER 3. RESOURCE ALLOCATION FOR TWO-HOP COMMUNICATION

WITH ENERGY HARVESTING CONSTRAINTS OVER FADING CHANNELS

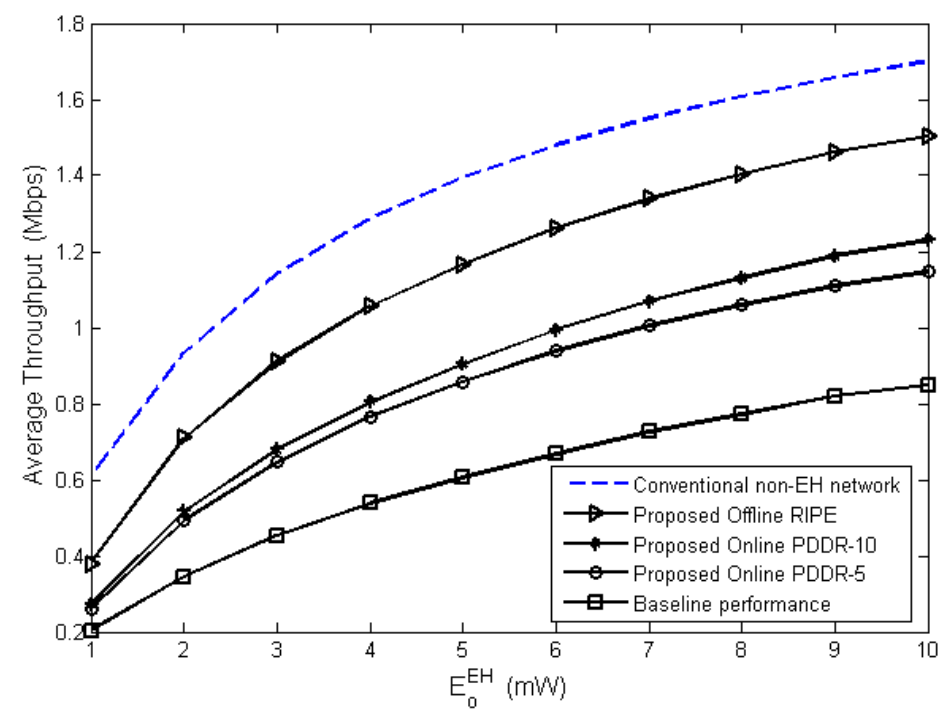

Figure 3.5: Simulation results of the throughput versus the source EH rate for a non-delay tolerant two-hop network under Rayleigh fading with $\bar{m}=5$.

bound of the short-term throughput for a conventional non-EH two-hop network over a Rayleigh fading channel.

Fig.3.6 shows the average throughput for the resource allocation schemes proposed for a delay tolerant network versus the average EH rate for the source $\left(E_{o}^{E H}\right)$ for $(K \rightarrow \infty)$ and with a constant relay peak transmit power $P_{M}^{R}=10 \mathrm{~mW}$ over a Rayleigh fading channel. It is quite clear that the throughput performance of the RID-AC algorithm has surpassed the performance of the RID-AF algorithm with average fading. Moreover, it can be noticed that the average throughput of the offline proposed RID-AC scheme increases sharply as $\left(E_{o}^{E H}\right)$ increases, whereas the average throughput of the online proposed RID-AF scheme increases exponentially as $\left(E_{o}^{E H}\right)$ increases. Fig.3.7 shows the average throughput versus $\kappa$ with different source average EH rate. It can be observed that the average throughput has a direct relationship with the scale factor, and the network gains the maximum throughput when $\kappa=2$. 
CHAPTER 3. RESOURCE ALLOCATION FOR TWO-HOP COMMUNICATION WITH ENERGY HARVESTING CONSTRAINTS OVER FADING CHANNELS

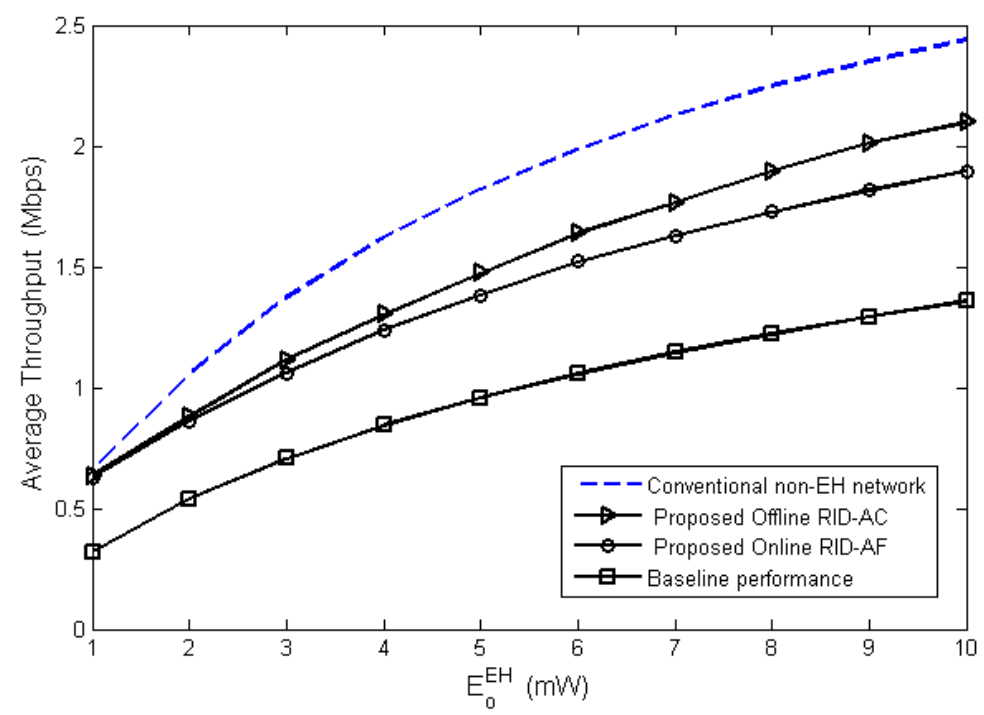

Figure 3.6: Simulation results of the throughput versus the source EH rate for a delay tolerant two-hop network over a Rayleigh channel with $\bar{m}=5$.

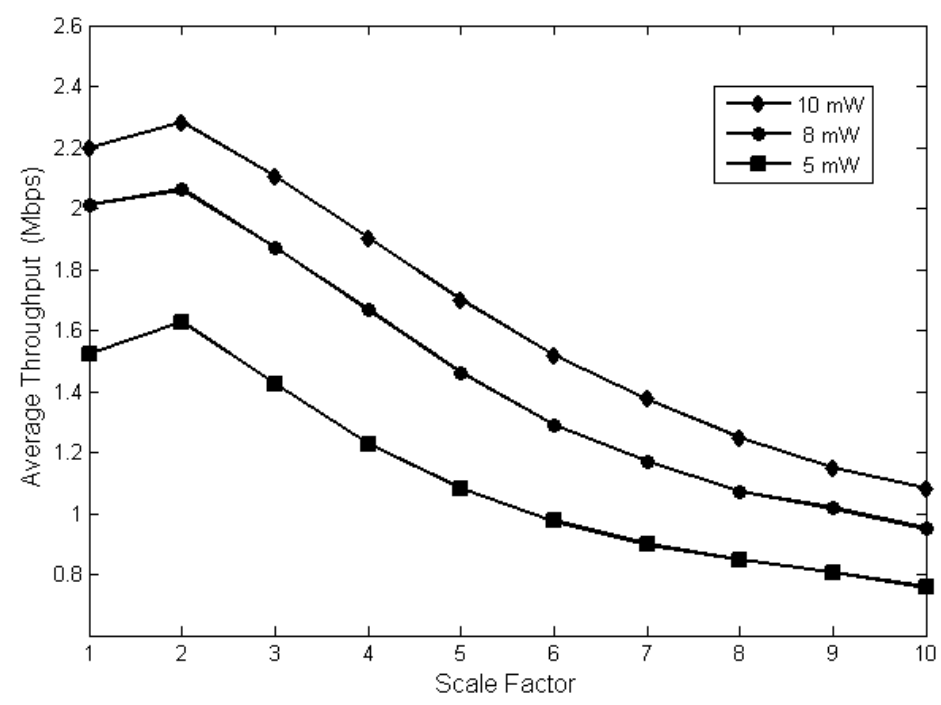

Figure 3.7: Average throughput versus $\kappa$ with $\left(P_{o}^{E H}=[5,8,10] \mathrm{mW}\right)$ under Rayleigh fading with $\bar{m}=5$.

\subsection{Chapter Summary}

This chapter investigates the resource allocation problem for a two-hop communication system with energy harvesting constraints on the source in a fading channel environment. The 
CHAPTER 3. RESOURCE ALLOCATION FOR TWO-HOP COMMUNICATION WITH ENERGY HARVESTING CONSTRAINTS OVER FADING CHANNELS

simple and elegant RGWF approach that solves the power allocation problem for a single-hop transmission is extended and modified to maximize the network throughput for a two-hop communication system. The importance of this representation is that it provides more insight into the problems and the solutions such that various wireless systems can be analysed. This chapter shows the advantage of adapting the RGWF algorithm for the throughput maximization problem under a Rayleigh fading channel. Moreover, two schemes that maximize the network performance from a throughput perspective are proposed for both delay tolerant and non-delay tolerant networks. Transmission scheduling time has been derived for the source and the relay based on RGWF-EH profile to obtain an efficient transmission policy. Numerical results illustrated that optimizing both transmission scheduling and power allocation result in gaining higher throughput. Moreover, simulations show that the proposed approach is simple, efficient and provides significant guidelines on network deployment and resource management in a green radio network with EH sources. 


\section{Chapter 4}

\section{SMDP-Based Resource Allocation for Wireless Networks with Energy Harvesting Constraints}

\subsection{Introduction}

Wireless communication powered by Energy Harvesting (EH) is a promising approach to prolong the lifetimes of wireless networks by eliminating the need for manual battery replacement while clean and renewable energy is approved. Moreover, it has become more desirable to reduce the world's energy consumption. However, the random nature of EH technology, i.e., energy arrives at random times and in random amounts, results in challenges to providing satisfactory communication performance in EH networks. Hence, the design of algorithms that handle the random and on-off energy arrivals is highly needed. This section investigates the energy resource allocation problem for EH networks, and pro-

Parts of Chapter 4 are presented and published at the IEEE Vehicular Technology Conference (VTC) in Toronto, Canada [88]. 
poses a resource allocation framework based on a Semi-Markov Decision Process (SMDP). The objective of the framework is to provide a solution for a throughput maximization problem in EH networks by maximizing the total long-term expected reward of the EH system. The system reward is derived by considering both the income and the cost of the EH wireless communications. The numerical results illustrate a significant expected reward performance gain over a Greedy scheme. Moreover, simulations illustrate that the proposed approach is efficient and provides important guidelines for network deployment and resource management in a green radio network with EH technology.

The design of EH communication systems has attracted considerable research attention recently. The throughput maximization problem for a single-hop network with EH constraints over a fading channel has been investigated in [12] and [56]. Authors in [82] revisited [56], and an efficient transmission policy was proposed that maximizes the throughput of a two-hop wireless network with EH constraints. In [89], the authors investigated the resource allocation problem in ultra-dense small cell networks with energy harvesting base stations. Failure probability minimization for wireless mesh networks with renewable energy suppliers has been addressed in [90]. On the other hand, considering the random nature of EH technology, the problem falls under a stochastic dynamic programming method [91]. Therefore, it is more realistic to utilize an optimization criterion that is dynamic in nature. Several papers formulate the EH networks using the Markov Decision Process (MDP), which is a mathematical framework that is utilized to analyze the system dynamics in an uncertain environment. Nevertheless, the main drawback of MDP is that action taken is time-event based. Due to the stochastic nature of the duration of successive decision-epochs in arriving energy and data, as well as the reliance on the costs of the decision-epochs duration, Semi-Markov Decision Process (SMDP), which is an event-driven process, is more relevant to wireless communication with EH technology.

In this Chapter, the focus is on the resource allocation problem for an online EH pointto-point communication system, and a resource allocation framework with an SMDP-based 
approach is proposed. Because of a random energy arrival process, the node must have a preplan to store enough energy to distribute the harvested energy to the incoming data requests

efficiently. The objective of the framework is to obtain the optimal decision for resource allocation on the EH wireless communication by maximizing the network throughput and reducing the service rejection probability. To the best of our knowledge, there is no existing work reported in the open literature that formulates the resource allocation problem of $\mathrm{EH}$ wireless networks as an infinite horizon SMDP-based problem. The main contributions of this chapter are summarized as follows: 1) a novel framework based on an SMDP approach is formulated for the EH wireless communication system with the objective of maximizing the throughput of a point-to-point transmission; 2) an optimal SMDP-based policy is proposed that is dynamically adaptive to the changing of the channel status and the varying of arriving harvested energy and data requests for scheduling decisions of the energy allocation.

The remainder of this chapter is organized as follows. In Section 4.2, the system model of EH technology is presented. The formulation of an SMDP-based approach for the energy allocation model is proposed in Section 4.3. Section 4.4 shows the implementation of the proposed policy in the proposed system model. Performance analysis is provided in Section 4.5, and chapter summary is given in Section 4.6.

\subsection{System Model}

\subsubsection{Model Description}

This work considers an EH technology for point-to-point wireless communication with a single EH transmitter and a single non-EH receiver over a fading channel in Fig. 4.1. The transmitter is equipped with an energy storage with finite capacity that can store at most $K_{\max }$ harvested energy units (EUs). Representation of relevant variables is adopted as discrete times. The list of important notations is given in Table 4.1.

Let $\lambda_{c}$ denote the average data arrival rate at the transmitter, where data is assumed 


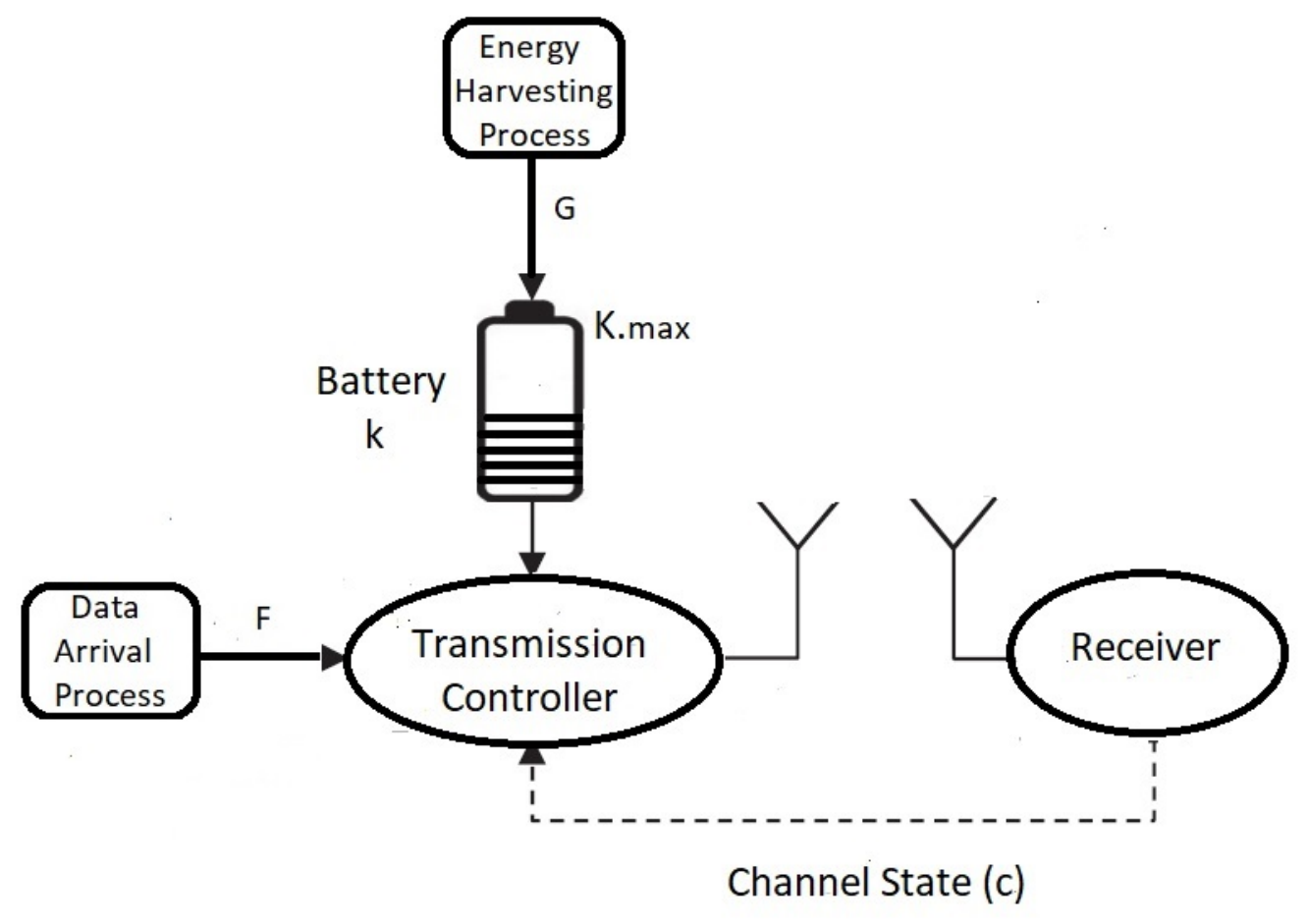

Figure 4.1: System block diagram.

to arrive with low arrival rate follows a Poisson distribution. Thus the transmission time of the services follows an exponential distribution with $\frac{1}{\mu}$ as the average transmission time with one allocated EU. Therefore, $\frac{\xi(n)}{\mu}$ is the transmission time with $n$ allocated EUs. Moreover, let $\lambda_{e}$ denote the arrival rate of EH into the source's energy buffer. We assume that EH arrival follows a Poisson distribution. Since the transmitter has a finite energy capacity, it must verify that the energy storage is neither too full nor empty to avoid buffer overflow or performance degradation, respectively. For simplicity, on the other hand, a three-state wireless channel model is considered that describes our point-to-point transmission in three possible fading states: good state, moderate (mod) state, and bad state, has been adopted. In particular, it is considered that the incoming packet that the source reports to the receiver are arriving at three pre-defined channel states, i.e., good, mod, and bad. Assuming $A_{c}$ 
CHAPTER 4. SMDP-BASED RESOURCE ALLOCATION FOR WIRELESS

NETWORKS WITH ENERGY HARVESTING CONSTRAINTS

Table 4.1: List of Notation

\begin{tabular}{|c|c|}
\hline Symbols & Description \\
\hline $\mathrm{K}_{\max }$ & Maximal amount of energy that the battery can accommodate \\
\hline $\mathrm{K}$ & Number of available EUs in the battery \\
\hline$\lambda_{c}$ & Average data arrival rate at the transmitter \\
\hline$\lambda_{e}$ & Average EH arrival rate at the transmitter's battery \\
\hline$\mu_{c}$ & Departure rate of the transmission that occupies one EU when the channel at state (c) \\
\hline$\xi(n)$ & Number of allocated EUs into a packet for transmission \\
\hline $\mathrm{A}_{c}$ & Event of arriving a packet when the channel at state (c) \\
\hline $\mathrm{G}$ & Event of arriving EU \\
\hline $\mathrm{F}_{n}$ & Event of completion a transmission that is assigned with $\mathrm{n}$ EUs \\
\hline $\mathrm{E}_{d}$ & Reward for transmitting a packet \\
\hline$\theta_{c}$ & Transmission time of the served service that occupies one EU at channel state $(\mathrm{c})$ \\
\hline$\beta$ & Price per unit time \\
\hline$\alpha$ & Continuous-time discounting factor \\
\hline
\end{tabular}

denotes the events of arriving a packet at the transmitter when the channel is at $c$ state, where $c \in(g, m, b)$ denotes good, mod, and bad wireless channel states, respectively. Let $\operatorname{Pr}_{g}, \operatorname{Pr}_{m}$, and $\operatorname{Pr}_{b}$ be defined as the probabilities of occurring events $A_{g}, A_{m}$, and $A_{b}$, respectively, where the sum of all the probabilities is one. Hence, the average arrival data rate $\bar{\lambda}_{c}=\sum_{\forall c} P r_{c} \lambda_{c}$. Certainly, the average gain of the good channel state is higher than that of the mod state and bad channel state. Thus, it permits transmission with less EUs using the same transmission parameters. We assume that the channel gain is block-fading so that channel gain remains the same for the whole duration of the decision-epoch, where channel condition is known at the beginning of the decision-epoch.

\subsubsection{Problem Formulation}

The decision-making procedure of the resource allocation problem is described as follows. When a new packet arrives, the controller determines whether to accept it or not according to the current channel states, and energy availability. If the data is accepted for transmission, the controller will assign the incoming packet with $n$ EUs, $n \in\{1,2, \cdots, N\}, N \leq K$, where $N$ 
is the maximal number of EUs that the source can provide for packet transmission, and $K$ is the number of available EUs in the transmitter's battery. The availability of the resources in the battery is varied based on the arrival of EH and the allocation of EUs into the incoming packet.

In this chapter, an online point-to-point EH communication system is formulated based on the SMDP-based approach. The objective of the formulation is to compute an optimal decision rule that maximizes the system benefits (long-term expected average system reward) and guarantees QoS in terms of network reliability by optimally allocating the arrival energy. The duration of a decision-epoch is a random variable and depends on the energy allocation decision. The control action is taken at the beginning of a decision-epoch, where it is continued up to the end of that interval. Each control action corresponds to a certain transmission power level, which is equivalent to the number of allocated EUs. On the other hand, once a transmission is accepted by the source node, then the completed rate to serve the packet is determined by the number of EUs given for that transmission and the channel states at the time when the packet arrives. The transmit time of a service is a reciprocal function of the number of EUs that is allocated to the packet. Therefore, the growing amount of EU allocation leads to completing the transmission faster.

\subsection{SMDP Formulation Of EH Technology}

As discussed earlier, the duration of the decision-epoch is not fixed since the time between successive control choices varies, where the epoch length depends on the current state and the choice of the action. The cost per decision-epoch relies on the time taken for the transitions from one state to another. Hence, the proposed problem shapes an SMDP problem, which is dynamic programming based. The Semi-Markov Decision Process problem can be modeled based on the following elements: system states, set of actions, reward model, Sojourn time, and transition probabilities, which are discussed below. 


\subsubsection{System States}

The system state of the resource allocation problem for EH technology $s$ can be characterized by the ongoing transmission with $n$ EUs, the available resources in the transmitter's battery, and the current event. Therefore, the state set $\mathcal{S}$ can be represented as

$$
\mathcal{S}=\left\{s \mid s=\left(s_{n}, K, \tilde{e}\right)\right\}
$$

where $s_{n}$ denotes the bitmap of allocating a transmitting packet with $n$ EUs and $\tilde{e}$ represents events in the set, where $\tilde{e} \in\left\{A_{c}, F_{1}, F_{2}, \ldots, F_{N}, G\right\}$. The arrival of a packet at $(c)$ channel states $\left(A_{c}\right)$; where $c \in\{g, m, b\}$, the completion of current transmission assigned with $n$ EUs $\left(F_{n}\right)$, and the arrival of one $\mathrm{EH}(G)$ are different events. In the beginning of each decisionepoch, at most one state is selected among all possible states. The overall objective is to optimize the online resource allocation that maximizes the end-to-end network throughput over Time $T$ for the proposed system model. Two points need to be emphasized. First, there exists some time instant when there is no energy allocated, i.e., the incoming data is dropped due to lack of available energy or a busy channel. Second, for the state selection in each decision-epoch, the system should take into consideration not only the throughput of this block but also the average throughput of the whole transmission period to obtain

a better overall performance. The source transmitting power can be computed according to the fundamental fact that in the total transmission period, a balance between the total harvested energy and the total consumed energy should be achieved from all the states.

\subsubsection{Set of Actions}

The decision process of the EH network determines its admission policy upon a packet arrival into the network. When an event occurs, the EH transmitter decides which action $a(s)$ must be taken from the action set $\mathcal{A}_{s}$ based on the current state $\mathbf{s}$, i.e., 


$$
\mathcal{A}_{s}= \begin{cases}-1, & \tilde{e} \in\left\{F_{1}, \ldots, F_{N}, G\right\} \\ \{0,1, \ldots, C\} . & \tilde{e}=A_{c}\end{cases}
$$

where $a(s)=-1$ represents either completing a transmission or harvesting new energy units. In both cases, no action is required except the transmitter's battery has to be updated. On the other hand, $a(s)=0$ represents an action of blocking an incoming data, where no energy shall be allocated, and $a(s)=n, n \in\{1,2, \ldots, N\}$ represents an action of accepting the incoming request with $n$ EUs. We assume that the transmitter's battery can receive energy packets during data transmission. To obtain the optimum resource allocation policy, a complete mapping from the states to the actions that maximizes the sum of all long-term expected rewards for each state $s$ must be found.

\subsubsection{Reward Model}

Given the system state and the corresponding action, the system reward $R(s, a)$ can be represented as

$$
R(s, a)=Q(s, a)-G(s, a)
$$

where $Q(s, a)$ and $G(s, a)$ refer to the instant income and the expected system cost, respectively, which are obtained by taking action $a(s)$ at state $s$. The income $Q(s, a)$ includes the improvement in system's satisfaction by admitting the transmitting; and the reduction in satisfaction rate by dropping the packet; and the expense of the transmission occupying the EUs. Therefore, the income can be shown as: 


$$
Q(s, a)= \begin{cases}0, & a(s)=-1, \\ & \tilde{e} \in\left\{F_{1}, \ldots, F_{N}, G\right\} \\ -E_{d}, & a(s)=0, \tilde{e}=A_{c} \\ E_{d}-\xi(n) \cdot \theta_{c} \cdot \beta, & a(s)=n, \tilde{e}=A_{c} \\ & n \in\{1,2, \cdots, C\}\end{cases}
$$

where $E_{d}$ refers to the cost or income evaluated by the change of system's satisfaction rate of rejecting or accepting a packet for transmission, respectively. $\theta_{c}$ denotes the transmitted time while the transmission is allocated one $\mathrm{EU}$ at channel state $(c) . \beta$ denotes the price per unit time whose unit is the same as the unit of $E_{d}$. Hence, the income of the EH wireless network can be written as:

The expected system cost $G(s, a)$ is defined as follows:

$$
G(s, a)=\tau(s, a) C(s, a),
$$

where $\tau(s, a)$ is the expected time from the exiting state to the next state when an action $a(s)$ is selected, and $C(s, a)$ is the service price of the expected time $\tau(s, a)$, which can be defined as the number of occupied EUs, shown as,

$$
C(s, a)=\xi(n) .
$$

\subsubsection{Sojourn Time}

The average expected time $\tau(s, a)$ is the time duration from the current event to others after selecting action $a(s)$. The average rate of an occurred event $\gamma(s, a)$, consequently, is the sum of all elements processes' rates from state $s$ to other states after selecting action $a(s) \cdot \gamma(s, a)$ 
and $\tau(s, a)$ can be computed as follows:

$$
\gamma(s, a)=\tau(s, a)^{-1}= \begin{cases}\lambda_{c}+\lambda_{e}, & \tilde{e} \in\{F, G\} \quad \text { or } \quad \tilde{e} \in\left\{A_{c}\right\}, a=0, \\ \lambda_{c}+\lambda_{e}+\frac{\mu_{c}}{\xi(n)} . & \tilde{e} \in\left\{A_{c}\right\}, a=n\end{cases}
$$

The expected instant reward $r(s, a)$ during $\tau(s, a)$ is determined according to the discounted reward model in [92], which is given by:

$$
\begin{aligned}
R(s, a) & =Q(s, a)-C(s, a) E_{s}^{a}\left[\int_{0}^{\tau} e^{-\alpha t} d t\right] \\
& =Q(s, a)-C(s, a) E_{s}^{a}\left[\frac{\left[1-e^{-\alpha \tau}\right]}{\alpha}\right] \\
& =Q(s, a)-\frac{C(s, a)}{\alpha+\gamma(s, a)},
\end{aligned}
$$

where $\alpha$ represents a continuous-time discounting factor.

\subsubsection{Transition Probability}

Next, the state transition probability $P\left(s^{\prime} \mid s, a\right)$ is defined as the probability that the system will be in state $s^{\prime}$ at the next decision epoch if an action $a(s)$ is selected at state $s$. Three cases are considered based on the event type $\tilde{e} \in\left\{A_{c}, F_{n}, G\right\}$ at current state $s$, where $A_{c} \in\left\{A_{g}, A_{m}, A_{b}\right\}$.

- For the given state $s=\left(s_{n}, K, A_{c}\right)$, the transition probability $P\left(s^{\prime} \mid s, a\right)$ to the next state $s^{\prime}$ can be obtained as

$$
P\left(s^{\prime} \mid s, a\right)= \begin{cases}\frac{P r_{c} \lambda_{c}}{\gamma(s, a)}, & a=0, s^{\prime}=\left(s_{n}, K, A_{c}\right) \\ \frac{\lambda_{e}}{\gamma(s, a)}, & a=n, s^{\prime}=\left(s_{n}, K+1-n, G\right) \\ \frac{\mu_{c}}{\xi(n) \gamma(s, a)}, & a=n, s^{\prime}=\left(s_{n}, K-n, F_{n}\right),\end{cases}
$$

where $P r_{c} \in\left\{P r_{g}, P r_{m}, P r_{b}\right\}$, and the summation of the three probabilities equals one. 
- For the given state $s=\left(s_{n}, K, F_{n}\right)$, the transition probability $P\left(s^{\prime} \mid s, a\right)$ to the next state $s^{\prime}$ can be obtained as

$$
P\left(s^{\prime} \mid s, a\right)= \begin{cases}\frac{P r_{c} \lambda_{c}}{\gamma(s, a)}, & a=n, s^{\prime}=\left(s_{n}, K-n, A_{c}\right) \\ \frac{\lambda_{e}}{\gamma(s, a)}, & a=-1, s^{\prime}=\left(s_{n}, K+1, G\right) .\end{cases}
$$

- For the given state $s=\left(s_{n}, K, G\right)$, the transition probability $P\left(s^{\prime} \mid s, a\right)$ to the next state $s^{\prime}$ can be obtained as

$$
P\left(s^{\prime} \mid s, a\right)= \begin{cases}\frac{P r_{c} \lambda_{c}}{\gamma(s, a)}, & a=n, s^{\prime}=\left(s_{n}, K-n, A_{l}\right) \\ \frac{\mu_{c}}{\xi(n) \gamma(s, a)}, & a=-1, s^{\prime}=\left(s_{n}, K, F_{n}\right) \\ \frac{\lambda_{e}}{\gamma(s, a)}, & a=-1, s^{\prime}=\left(s_{n}, K+1, G\right) .\end{cases}
$$

Then, the maximal long-term discounted reward can be gained according to the discounted reward model defined in [93] and can be written in the Bellman equation form

$$
v(s)=\max _{a \in \mathcal{A}_{s}}\left[R(s, a)+\lambda \sum_{s^{\prime} \in \mathcal{S}} P\left(s^{\prime} \mid s, a\right) v\left(s^{\prime}\right)\right],
$$

where $\lambda=\frac{\gamma(s, a)}{\alpha+\gamma(s, a)}<1$ to ensure the convergence of the calculation. In addition, the state transition probability after uniformization $\tilde{p}\left(s^{\prime} \mid s, a\right)$ can be expressed as

$$
\tilde{p}\left(s^{\prime} \mid s, a\right)= \begin{cases}1-\frac{\left[1-P\left(s^{\prime} \mid s, a\right)\right] \gamma(s, a)}{L}, & s^{\prime}=s \\ \frac{P\left(s^{\prime} \mid s, a\right) \gamma(s, a)}{L}, & s^{\prime} \neq s .\end{cases}
$$

where $L=\lambda_{c}+\lambda_{e}+\frac{\mu_{c}}{\xi(n)}$. 


\subsection{Energy Allocation Scheme By SMDP}

By finding a complete mapping from the states to the actions, the optimal resource allocation policy can be obtained. Based on the above transition probabilities and the reward model in (4.8), the maximum long-term discounted reward of the state $s$ for our SMDP-EH approach can be formulated as the discount reward model defined in a Bellman equation as shown in (4.12). The expected real-time reward has to be unitized to uniform the continuous-time Markov decision process. The normalized long-term expected reward $\tilde{v}(s)$ for the proposed adaptive optimal energy allocation of a wireless EH network is stated as:

$$
\tilde{v}(s)=\max _{a \in \mathcal{A}}\left[\tilde{R}(s, a)+\tilde{\lambda} \sum_{s^{\prime} \in S} \tilde{p}\left(s^{\prime} \mid s, a\right) \tilde{v}\left(s^{\prime}\right)\right]
$$

where the unitized reward function $\tilde{R}(s, a)$ and the unitized parameter $\tilde{\lambda}$ are determined as following

$$
\begin{aligned}
\tilde{R}(s, a) & =R(s, a) \frac{\alpha+\gamma(s, a)}{\alpha+L} \\
\tilde{\lambda} & =\frac{L}{(L+\alpha)}
\end{aligned}
$$

The objective of determining $\tilde{v}(s)$ is to choose an action that maximizes the right hand side expression. It is noted that the proposed model is the infinite SMDP with finite states, which provides a stationary policy. Hence, the optimization problem given by (4.14) can be computed by applying the policy iteration approach [91] as summarized in Algorithm 4. The output $\left(P_{\text {opt }}\right)$ is the optimum decision policy of the system.

In the beginning, both $\tilde{v}(s)$ and $P_{\text {opt }}(s)$ are initiated to be zero for each state $s$. For each state $s, \tilde{v}(s)$ and $P_{\text {opt }}(s)$ are repeatedly calculated until the value of $\tilde{v}(s)$ for every state $s$ equals the related $\tilde{v}^{\prime}(s)$ in the previous iteration, which indicates that the convergence is reached. The output $P_{\text {opt }}(s)$ for all states is the decision policy of the system, which results in obtaining the maximum discounted reward. 


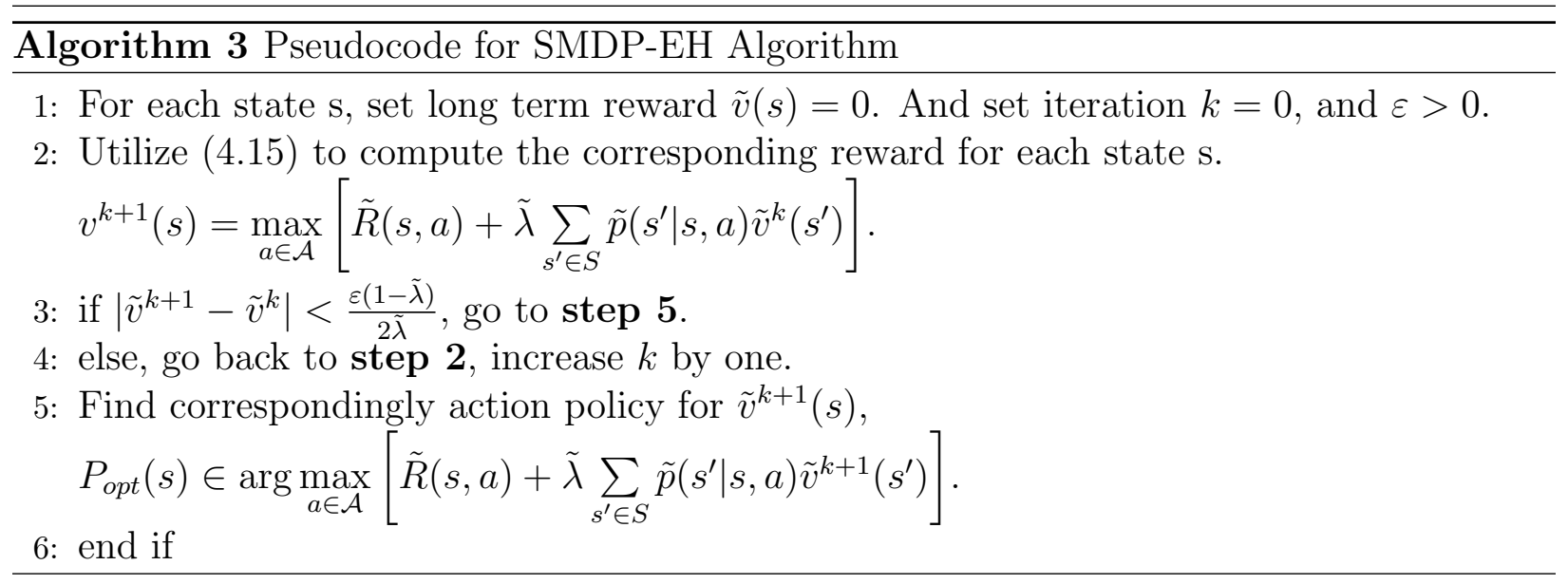

\subsection{Performance Evaluation}

In this section, simulation results are provided to evaluate the performance of the proposed SMDP adaptive energy allocation approach for an EH network. The proposed policy is compared with with Greedy policy [94] under various settings. In Greedy policy, the admission control always assigns the maximum number of resources that the system can afford to any data that arrives at any channel states. Our simulation is developed using an event driven method in MATLAB. The simulation runs for $100 \mathrm{sec}$, and it is repeated ten times to obtain the same average value as the simulation results for the convergence. The maximum number of allocated EUs to a packet is $N=3$. Specifically, a packet can be provided with none, one, two, or three EUs at most, which relies on the availability of resources and the overall system reward. The default values of other parameters used in the system model, except as otherwise noted, are as follows: three pre-defined channel states in the source-destination link are considered. Although the transmitter has a single data arrival rate $\lambda_{c}$, the probabilities that arriving data events occur when the channel is in a good, mod, or bad state are defined as $\operatorname{Pr}_{g}=0.3, P r_{m}=0.4$, and $P r_{b}=0.3$, respectively. The average departure rate of a service depends on the channel states when data arrives and the number of allocated EUs. The energy arrival rate is $\lambda_{e}=1$, the reward for transmitting a packet is $E_{d}=2$, the maximal size of the energy capacity is $K_{\max }=10, \beta=3$, and the discount factor $\alpha$ is 0.15 . 


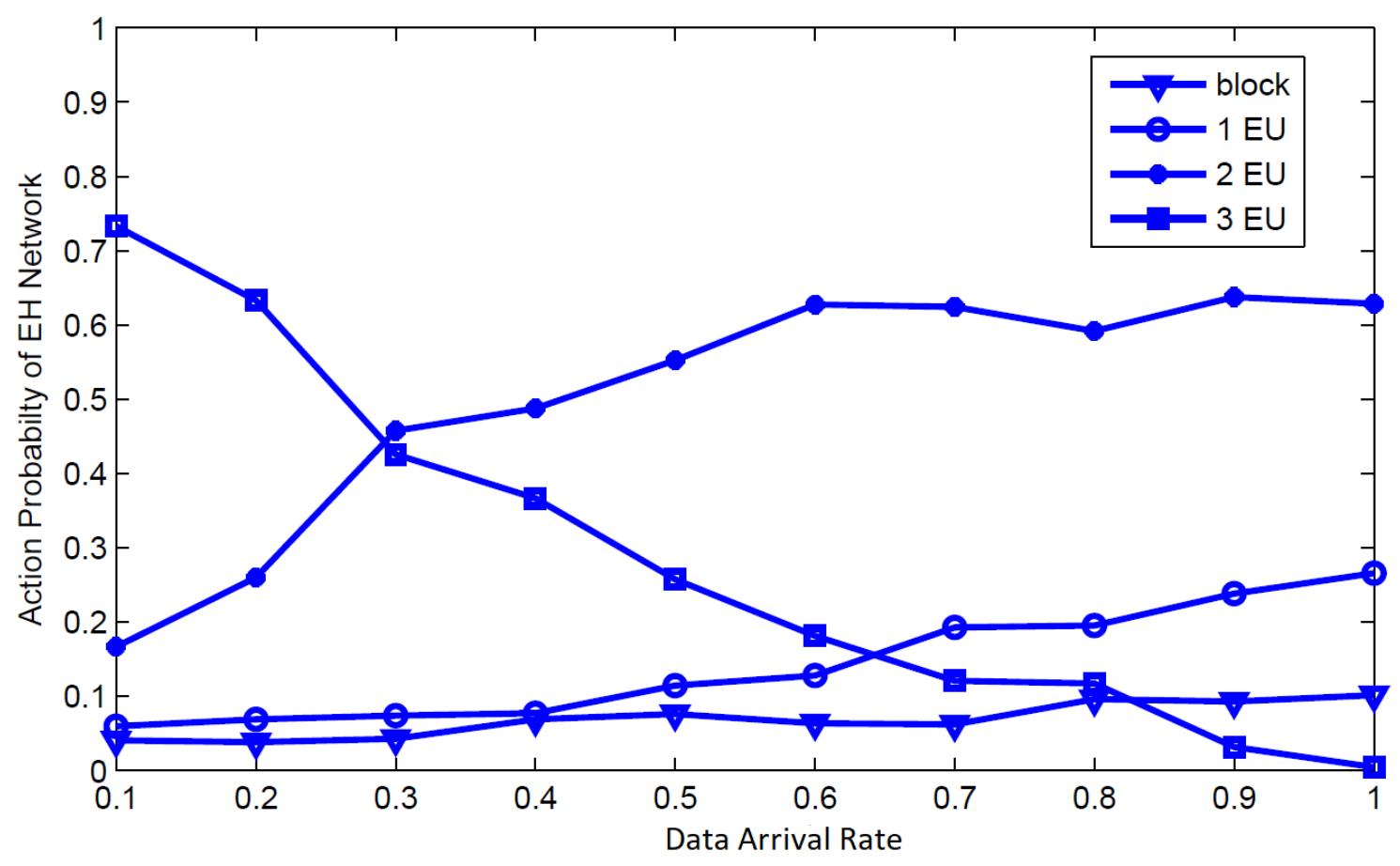

Figure 4.2: Average probability of action under various $\lambda_{c}$.

Fig. 4.2 shows the average action probabilities of the EH network various data arrival rates $\lambda_{c}$. It is noticeable that most requests are allocated to $3 \mathrm{EUs}$ when the data arrival rate is relatively very low, whereas the policy trends to perform the action of assigning less EUs when the data arrival rate starts increasing to adapt more transmission. In other words, the system avoids experiencing a shortage of energy by assigning services with minimum EUs in the case of high traffic.

To further verify the performance of our proposed SMDP-based resource allocation scheme, the blocking probability, as well as the overall system reward, are compared with Greedy policy over various data arrival rates, $\lambda_{c}$. In Fig. 4.3, the system reward of the EH network under variable data arrival rates is shown. As expected, it can be observed that the performance of our proposed scheme is superior to the Greedy scheme for all $\lambda_{c}$. Moreover, it is clear that the gap of the overall reward between the proposed algorithm and the Greedy algorithm is expanded with the increasing of the data arrival rates. 


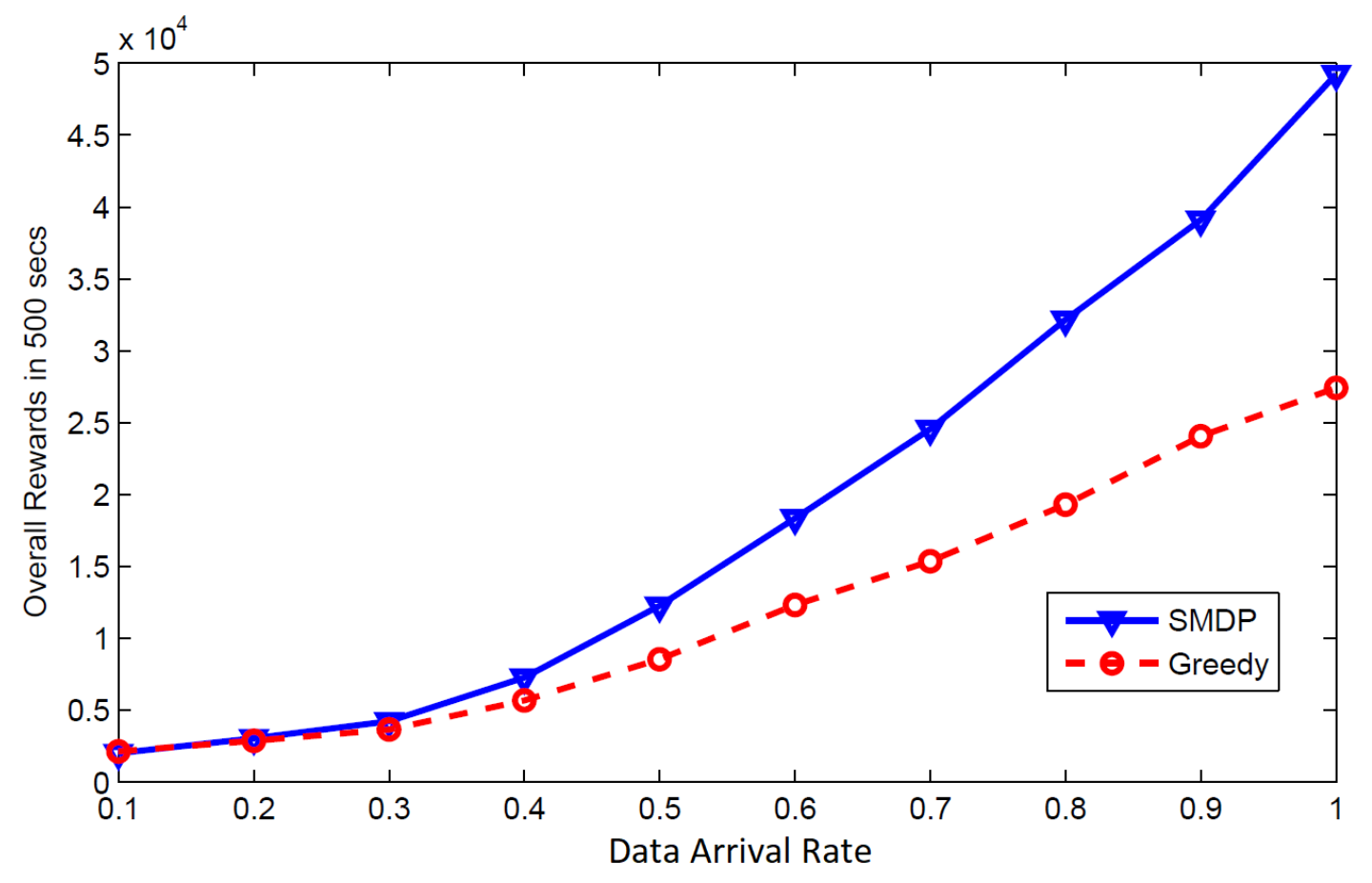

Figure 4.3: Overall reward under various $\lambda_{c}$.

A similar trend is observed in Fig. 4.4, which displays how the blocking probability grows with increasing data arrival rates. While the blocking probability increases slowly due to the high volume of data arriving for our proposed SMDP approach, the probability grows progressively more in the Greedy scheme. The reason the proposed algorithm outperforms the Greedy function is because of the inability of the Greedy policy to control the rejection rate when the data arrival rate is high, or the stored energy is low, while our scheme always maintains the blocking rate by optimally distributing the resource.

\subsection{Chapter Summary}

This chapter considers a point-to-point communication system with energy harvesting constraints over a fading channel, where a resource allocation framework based on a SemiMarkov Decision Process (SMDP) is proposed. The objective of the framework is to provide 


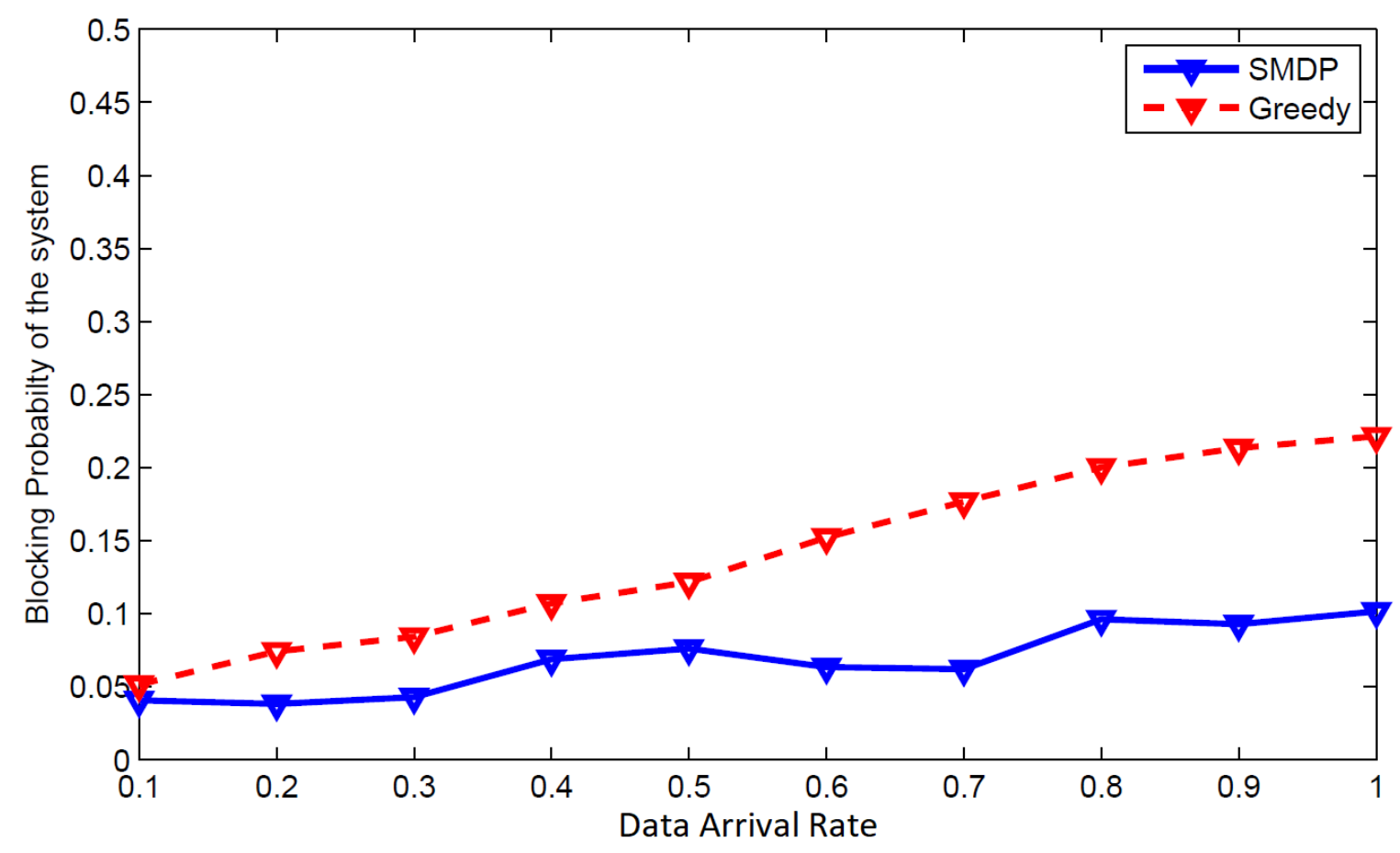

Figure 4.4: Blocking probability under various $\lambda_{c}$.

a solution for a throughput maximization problem in EH networks by maximizing the total long-term expected reward of the EH system. The system reward is derived by considering both the income and the cost of the $\mathrm{EH}$ wireless communications. The numerical results illustrate a significant expected reward performance gain over a Greedy scheme. Moreover, simulations illustrate that the proposed approach is efficient and provides important guidelines for network deployment and resource management in a green radio network with EH technology. 


\section{Chapter 5}

\section{Cross-layer Adaptive Transmission Techniques for EH Systems over Fading Channels}

\subsection{Introduction}

Energy harvesting (EH) technology is an important enabling technique for future wireless communication to meet the demand for self-organizing networks. Adaptive modulation, on the other hand, is crucial to meet the demand for high data rate transmission. Moreover, providing energy harvesting capability to wireless devices enables the nodes to continually acquire energy from renewable resources, which leads to prolonged lifetimes for wireless networks. Although Energy harvesting technology brings new dimensions to the wireless communication problem, the intermittency and randomness of arriving energy are still vast concerns. Besides self-sustainability and virtually perpetual operation compared to network lifetimes currently limited by conventional batteries, EH technology is expected to reduce the use of conventional energy and accompanying carbon footprint to reduce the worlds energy consumption, especially by increasing the number of communication devices, which will 
double or triple by 2050 [1]. Hence, wireless communication powered by energy harvesting is a promising approach to prolong the lifetimes of wireless networks. Traditional energy harvesting technology relies on natural energy sources (like solar, wind, vibration, etc.), which have certain characteristics. EH technology has recently emerged as the key solution to many problems related to the wasting of energy due to radio transmissions [2] and it is expected to significantly improve network energy efficiency. Besides this, it maximizes the network longevity by eliminating manual battery replacement while clean and renewable energy are approved. As a matter of fact, scavenging ambient energy and utilizing the free, renewable energy wisely will lead to opening a vast opportunity for green communication $[3,4]$. Despite the advantages provided by EH technology, the random nature of EH constraints, i.e., energy arrives at random times and in random amounts, results in challenges to providing satisfactory communication performance in EH networks. Hence, designing algorithms to handle the random and on-off energy arrivals is highly desirable. Moreover, decision making plays a crucial role in wireless networks with EH technology due to the randomness in the stochastic energy arrival process. Because of the time-varying fading of wireless channels and the randomness of arriving energy as well as data, the decision about the transmission rate and power depends primarily on channel conditions, data buffer status, and energy capacity status. Practical systems, data buffers and energy capacity are equipped with finite storage; therefore, optimal channel-adaptive schemes may not be enough to optimize the system as a whole, and buffers should be taken into consideration. Because of the randomness of arrival data and energy, as well as the varying of channel gains, exact scheduling policies cannot be determined using statical optimization techniques. Since the packet scheduling problem is inherently dynamic in nature, the problem falls under the stochastic dynamic programming. Therefore, it is more realistic to utilize an optimization criterion that is dynamic in nature. Several papers formulate the EH networks using the Markov decision process (MDP), which is a mathematical framework that analyzes the system dynamics in uncertain environments. Nevertheless, the main drawback of the MDP approach is that decisions taken are time-based. 
On the other hand, due to the stochastic nature of the duration of successive decision-epochs in arriving energy as well as data, and the reliance on the costs of the decision-epochs duration, the semi-Markov decision process (SMDP), which is event-based, is more relevant to wireless communication with $\mathrm{EH}$ technology. In this chapter, the cross-layer variable-rate variable-power adaptive modulation scheme is investigated for an online point-to-point EH communication systems based on channel state, data buffer state and battery state to maximize the network throughput while minimizing the dropped packets probability and data buffer delay. Due to the random arrival of the harvested energy, the node must have a preplan to store enough energy to efficiently distribute the arrival energy for data transmission. Our previous work [88], proposed a resource allocation framework of a point-to-point wireless communication system with EH constraints based on SMDP approach that maximizes the network throughput by considering channel adaptation only. Since the assignment of constellation only depends on the channel gain, this scheme may give an upper bound of the achievable physical layer performance by considering two assumptions: the buffer is infinite, and has data all the time to transmit with any chosen constellation. For practical wireless networks, adapting packet transmission with channel condition as well as by taking buffer status into consideration is crucial. Overall, the goal of the adaptation is to stabilize the system, by achieving maximum throughput while reducing dropping probabilities and minimizing buffering delay. Moreover, because of the finiteness of the data buffer, the calculation of delay and overflow is dynamic in nature, and therefore, a cross-layer adaptive data rate scheme is proposed that selects power constellation dynamically depending on the channel state as well as on the data buffer and the battery states. Both BER requirements at the physical layer, and delay and overflow requirements at the data link layer are satisfied. To the best of our knowledge, there is no existing work reported in the open literature that formulates the resource allocation problem of $\mathrm{EH}$ wireless networks as an infinite horizon SMDP-based problem under data buffer and overflow constraints.

The design of EH communication systems has generated much research activity with 
respect to modern wireless technology. The throughput maximization problem for a singlehop with EH constraints over a fading channel has been investigated in [12] while authors in [56] solved the same problem by proposing a low-complex and optimal transmission policy called recursive geometric water-filling (RGWF). The cooperative transmissions, mainly for the two-hop wireless communication systems with EH technology have been studied recently, but only for some special cases. The authors in [69] proposed an optimal transmission policy for the two-hop system model in multiple $\mathrm{EH}$ packets arriving at the relay. In [68], the throughput maximization problem for the two-hop communication system in the case of an EH source with two energy arrivals has been solved using cumulative curve algorithm. In [82], RGWF algorithm was adopted to maximize throughput of the two-hop EH systems. In [89], the authors investigated the resource allocation problem in ultra-dense small cell networks with energy harvesting base stations. Failure probability minimization for wireless mesh networks with renewable energy suppliers has been addressed in [90].

On the other hand, numerous system models have been formulated based on the SMDP approach, such as mobile cloud computing networks, vehicular cloud computing networks, wireless networks and cognitive vehicular networks. The authors in [95] showed how to manage the cloud resources, i.e., virtual machines, across multiple cloud domains to support continuous cloud service based on SMDP, where the work captures the dynamic arrivals and departures of resource requests for decision making of their resource allocation. In [96], the authors proposed a joint multi-resource allocation framework for the same system model in [95] based on SMDP, where the objective of the framework is to obtain the optimal decision of computing and wireless bandwidth resource allocation among multiple mobile users by maximizing the overall rewards (i.e., low service rejection probability and short service time). The authors in [97] proposed an optimal computation resource allocation scheme to maximize the total long-term expected reward of the vehicular cloud computing system based on SMDP. The system reward is derived by taking into account both income and cost of the proposed system as well as the variability feature of the resources. In [88], 
a novel framework based on SMDP approach is formulated for a point-to-point wireless communication with EH constraints. The objective of the framework is to dynamically adapt to the random nature of the energy arrival and the change of the channel fading in order to make optimum decisions in terms of packet transmission. From a cognitive vehicular networks perspective, the authors in [98] captured the dynamic of vehicle users mobility and the change in cognitive band availability, where the joint resource allocation problem is formulated as SMDP.

The main contributions of this chapter are summarized as follows:

- investigate the capability of employing SMDP-based policy optimization for an adaptive $\mathrm{EH}$ wireless communication system with the objective of maximizing network throughput and minimizing the dropping probability and data buffer delay.

- a novel framework based on the SMDP approach is formulated for EH wireless communication systems with the objective of maximizing network throughput by optimally allocating the resource while maintaining minimal buffering delay and packet overflow;

- a SMDP-based dynamic programming technique is proposed to dynamically adapt to the change of the channel and/or buffers' states that optimally satisfy the BER requirements at the physical layer, and the overflow requirements at the data-link layer.

The remainder of this chapter is organized as follows. The system model and model description are presented in Section 5.2. In Section 5.3, the SMDP Formulation of the Cross-Layer Scheduling Problem for EH Technology is provided. Section 5.4 shows the implementation of the proposed policy to our proposed system model. Performance analysis is provided in Section 5.5, and concluding remarks are given in Section 5.6. 


\subsection{System Model}

\subsubsection{Model Description}

This work considers an EH technology for a point-to-point wireless communication system over fading channels with a single EH transmitter and a single receiver. The transmitter is equipped with finite energy capacitor $K_{\max }$ and finite data buffer $D_{\max }$ as shown in Fig. 5.1. It is assumed that the point-to-point transmission is represented as radio frames, where a radio frame consists of multiple time-slots. The list of important notations of this chapter is given in Table 5.1.

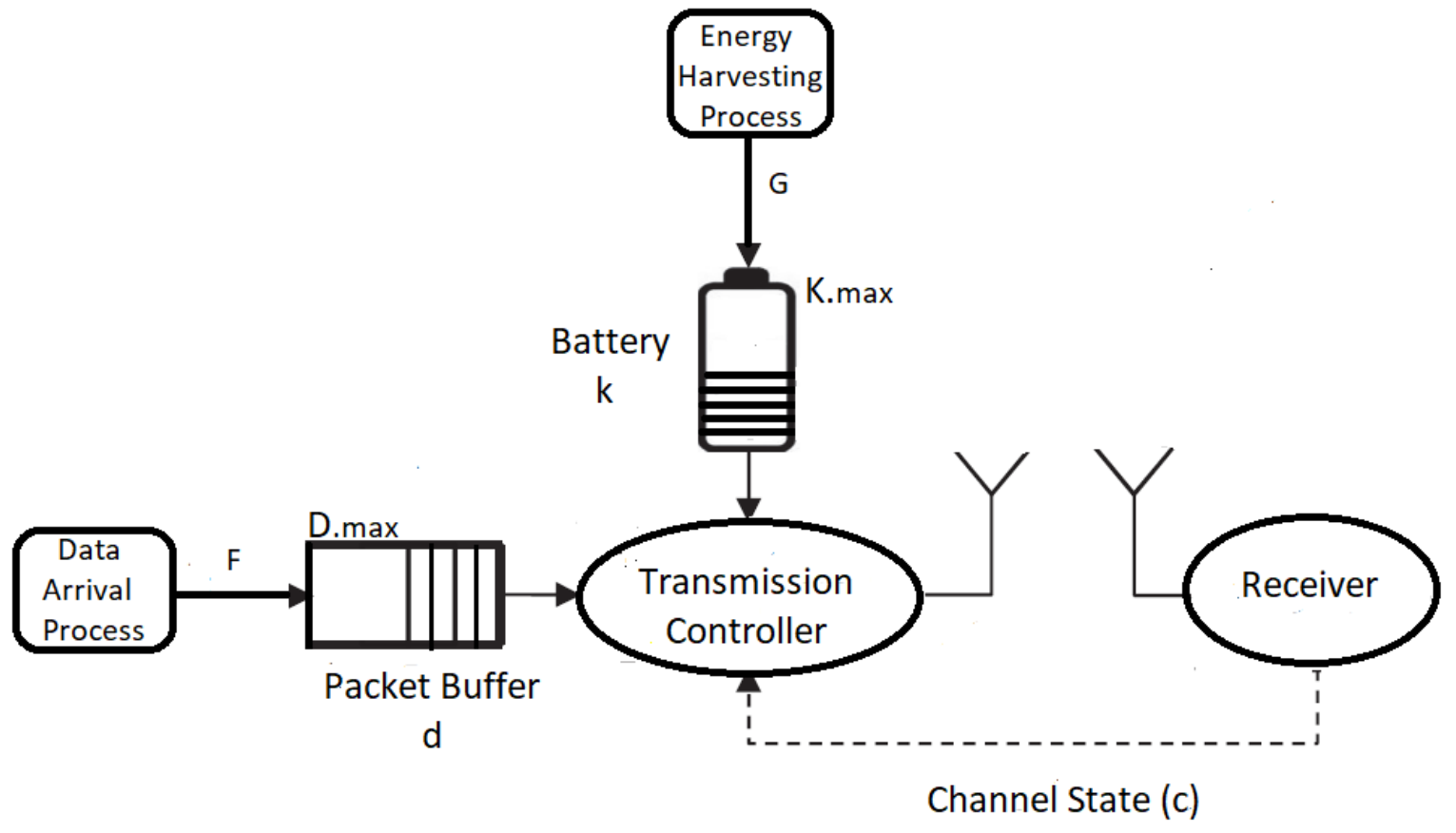

Figure 5.1: System block diagram.

Let $\lambda_{c}$ denote the average data arrival rate at the transmitter, where data is assumed to arrive with high arrival rate follows a Poisson distribution. Similarly, we assume that the transmission time of the service follows an exponential distribution with $\frac{1}{\mu_{c}}$ as the average transmission time with one EU when channel at $c$ state. Therefore, $\frac{\xi(n)}{\mu}$ is the transmission 
Table 5.1: List of Notation

\begin{tabular}{|c|c|}
\hline Symbols & Description \\
\hline $\mathrm{K}_{\max }$ & Maximal amount of energy that the battery can accommodate \\
\hline $\mathrm{D}_{\max }$ & Maximal number of packets that the buffer can accommodate \\
\hline $\mathrm{K}$ & Number of avaliable EUs in the battery \\
\hline $\mathrm{D}$ & Number of stored packets in the buffer \\
\hline$\lambda_{c}$ & Average data arrival rate at the transmitter's buffer \\
\hline$\lambda_{e}$ & Average EH arrival rate at the transmitter's battery \\
\hline$\mu_{c}$ & Departure rate of the transmission that occupies one EU when the channel at state (c) \\
\hline$\xi(n)$ & Number of allocated EUs \\
\hline $\mathrm{N}_{s}$ & Number of transmitted symbols in a time-slot \\
\hline $\mathrm{N}_{p}$ & Number of bits in Packet \\
\hline$w$ & Number of packets taken from the buffer \\
\hline $\mathrm{R}$ & Adaptive modulation rate in bits/symbol \\
\hline $\mathrm{G}$ & Event of arriving new EU \\
\hline $\mathrm{F}$ & Event of arriving new packet \\
\hline $\mathrm{C}_{c}$ & Event of completion transmission and starting a new transmission at channel state $(\mathrm{c})$ \\
\hline $\mathrm{P}_{e}(\gamma)$ & Instantaneous BER with received SNR $(\gamma)$ \\
\hline $\mathrm{P}_{T}$ & Transmission power \\
\hline$\alpha$ & Continuous-time discount factor \\
\hline
\end{tabular}

time with $n$ allocated EUs. Moreover, let $\lambda_{e}$ denote the average arrival rate of EH at the transmitter's battery. It is assumed that the EH arrival rate follows a Poisson distribution. The processing units are packet and block at the higher layer and at the physical layer respectively. A point-to-point transmission block consists of multiple symbols, and a packet is made up of multiple information bits. Packets from the higher layer application are stored in a finite sized buffer at the transmitter's data buffer. Based on the information of channel state, data buffer state and energy capacitor state, the scheduler chooses a particular action $u \in \mathcal{U}$, which is equivalent to the selected modulation constellation. The controller unit takes a corresponding number of packets from the buffer and modulates it with the chosen modulation scheme into symbols for transmission over a Rayleigh fading channel. At the receiver, the symbols are demodulated into bit streams, bit streams are mapped into the packets, and packets get stored in the receiver buffer. Finally, packets are pushed upwards 
to the higher layer from the receiver buffer. It is assumed that the discrete duration of each time-slot corresponds to a frame of $N_{s}$ channel uses (number of symbols transmitted in a time-slot). Depending on the scheduler decision, a frame may have a different number of packets in different time-slots. Let $w$ denote the number of packets taken from the buffer for transmission and $R$ is the adaptive modulation rate in bits/symbol. The relation between number of transmitted packets and modulation rate is given by,

$$
w=\left(\frac{N_{s}}{N_{p}}\right) R
$$

where $N_{p}$ is the packet size in bits.

\subsubsection{Channel Model}

The wireless channel in the analyzed EH technology system is assumed to be ergodic flat fading, obeying a Rayleigh distribution. The probability density function of power gain for the Rayleigh fading channel is described by a exponential distribution [99],

$$
f(\gamma)=\frac{1}{\bar{\gamma}} \exp \left(-\frac{\gamma}{\bar{\gamma}}\right), \text { for } \gamma \geq 0
$$

where $\bar{\gamma}=\mathbf{E}\{\gamma\}$ is the average received channel power gain. We model the Rayleigh fading channel with a first order Markov model, which is described with a set of channel states $\mathcal{C}=\left\{c_{1}, c_{2}, \cdots, c_{C}\right\}$ and a matrix of transition probabilities among states $\mathcal{P}=\left[\operatorname{Pr}_{c_{i}, c_{j}}, 1 \leq\right.$ $i, j \leq C]$, where $\mathrm{C}$ is the number of finite non-overlapping channel states and $\operatorname{Pr}_{c_{i}, c_{j}}$ is the probability of transition from state $c_{i}$ to state $c_{j}$, i.e., $\operatorname{Pr}_{c_{i}, c_{j}}=\operatorname{Pr}\left(c_{j} \mid c_{i}, 1 \leq i, j \leq C\right.$. Let $\Gamma=\left\{\gamma_{0}, \gamma_{1}, \cdots, \gamma_{C}\right\}$ denote the corresponding set of received SNR thresholds in increasing order, where $\gamma_{0}=0, \gamma_{i}<\gamma_{i+1}$ and $\gamma_{C}=\infty$. Then the channel is said to be in state $c_{i}$ if $\gamma_{i-1} \leq \gamma \leq \gamma_{i}$. This work considers a $C$-state wireless channel model that describes the point-to-point transmission in $C$-possible fading states: $c \in\left\{c_{1}, c_{2}, \cdots, c_{C}\right\}$. 


\subsubsection{Battery Model}

It is assumed that the transmitter is equipped with a finite energy capacitor that can hold at maximum of $K_{\max }$ EUs. Let $\mathcal{K}=\left\{k_{0}, k_{1}, \cdots, k_{K}\right\}$ denote the capacitor state space in terms of EU occupancy, where $k_{j}$ corresponds to $j \in\{0,1, \cdots, K\}$ EUs. The number of EUs in the buffer is determined dynamically based on battery status, energy consumption, and new harvested energy. The dynamics of the capacitor occupancy from the current state to next state is given by,

$$
k_{j}=\min \left\{\max \left(k_{0}, k_{i}-\min \left\{P_{T}, k_{i}\right\}+g\right), K_{\max }\right\}
$$

where the transmitting power $P_{T}$ must be smaller or equal to the current available energy $k_{i}$, and $g \in\{0,1, \cdots, G\}$ denotes the arrival of EUs at the transmitter's battery.

\subsubsection{Data Buffer Model}

The transmitter utilizes its data buffer to store the arrival packets. Let $\mathcal{D}=\left\{d_{0}, d_{1}, \cdots, d_{D}\right\}$ denote the data buffer state space in terms of buffer occupancy, where $d_{i}$ corresponds to $i \in\{0,1, \cdots, D\}$ packets in the buffer. The number of packets in the buffer at each decisionepoch is determined dynamically based on the current buffer state, transmitted packets, and new incoming traffic. It can be expressed as follows,

$$
d_{j}=\min \left\{\max \left(d_{0}, d_{i}-w+f\right), D_{\max }\right\}
$$

where $f \in\{0,1, \cdots, F\}$ denotes the number of packets that arrive at the data buffer, and $w \in\{0,2, \cdots, W\}$ denotes the packets that are taken from the buffer for transmission. It is taken into account that the maximum number of packets that can be transmitted is limited by the number of available packets in the data buffer.

With respect to an action at a particular state $a(s)$, each action corresponds to a pair 
of energy allocation and transmission rates as discussed in the next section. It is assumed that all packets arriving in any given time-slot cannot be transmitted immediately and can only be transmitted in the next time-slot or later. There are two other considerations that are made: (i) when the buffer has fewer packets than the number of packets that could be transmitted $d_{i}<w$ and (ii) when the buffer's empty space is less than the number of bits arriving, $d_{D}+w-d_{i}<f$. In the first case, the controller can take no more than the maximum number of bits in the buffer; therefore, the minimum buffer state could be $d_{0}$. In the second case, the transmitter can accommodate $d_{D}+w-d_{i}$ packets in the buffer and additional arriving packets will be dropped. Hence, the maximum buffer state is $d_{D}$.

\subsection{SMDP Formulation of EH Cross-layer Adaptive Transmission}

As discussed earlier, the duration of the decision-epoch is not fixed since the time between successive control choices varies, where the epoch duration depends on the current state and the choice of the action. The cost per decision-epoch relies on the time taken for the transitions from one state to another. Hence, the proposed problem constitutes a SMDP problem, which is dynamic programming based.

\subsubsection{Problem Description}

Maximizing the supportable data rate while minimizing power, delay and/or overflow simultaneously is difficult to manage. The reason is that to minimize the buffer delay and/or buffer overflow, high power is required for transmission with high order modulation and vice versa. In this chapter, the objective is to design a cross-layer scheduler for an online point-to-point EH wireless network that optimally allocates energy and transmission rates while being adaptive to the change of both physical layer (i.e., channel state) and data link layer (i.e., battery and data buffer states) such that the overflow and the queueing delay are 
optimized while the network throughput is guaranteed. The semi-Markov decision process problem can be modelled based on the following tuple $\left\{\mathcal{S}, \mathcal{A}_{s}, \mathcal{W}, \mathcal{T}_{s}, \mathcal{P}\right\}$, which corresponds to the system states, set of actions, reward model, sojourn time, and transition probabilities, as discussed below:

\section{System States}

The system state of the cross layer power allocation and adaptive modulation scheme for $\mathrm{EH}$ technology $s$ can be characterized by the ongoing transmission with $n$ EUs and corresponding adaptive rate, channel state, battery state, buffer state, and current event as represented below

$$
\mathcal{S}=\left\{s \mid s=\left(s_{n, w}, K, D, \tilde{e}\right)\right\}
$$

where $s_{n, w}$ denotes a bitmap of the ongoing transmission that is allocated with $n$ EUs $n \in\{0,1, \cdots, N\}$ and $w$ packets that are taken from the data buffer $w \in\{0,2, \cdots, W\}$. In addition, $\tilde{e}$ represents the event in the event sets, where $\tilde{e} \in\left\{G, F, C_{c}\right\}$. The arrival of harvested energy $(\mathrm{G})$, the arrival of data packets $(\mathrm{F})$, and the completion of the ongoing transmission and starting of a new transmission at channel gain (c) are different events.

At a particular event $\tilde{e}$, the scheduler first determines the status of the buffers and the channel, and then chooses an action dynamically to optimize average transmission power, delay and overflow. The objective of the scheduler is to optimize the online resource allocation that maximizes the network throughput under the constraints on the average buffer delay and packet overflow for the proposed system model. Since the nature of the problem is dynamic, it falls into the general category of stochastic dynamic programming problems. The duration of a decision-epoch is a random variable and depends on the decision of the scheduler. The control action is taken at the beginning of a decision-epoch, and it continues up to the end of that interval. Similar to Chapter 4, a transmitter's battery can harvest energy during data transmission. 


\section{Set of Actions}

In an adaptive power allocation and modulation scheme, each action in the action set of the adaptive transmission scheme has a two-to-one mapping between the energy allocation and the transmission rate on one hand, and the number of transmitted packets on the other hand.

At the start of a particular time-slot, the adaptive controller decides the action $a$ at state $s$ to be taken depending on the data buffer state, energy capacity state as well as channel state. Assume that there are a total of $\mathcal{A}_{s}$ actions available at state $s$ and $\mathcal{A}_{s}=\left\{a_{0,0}, a_{1,2}, a_{1,4}, \cdots, a_{n, w},\right\}$ to be the set of all available actions, where the first index $n$ represents the number of allocated EUs and the second index $w$ represents the number of transmitted packets. Hence, when an event occurs, the controller chooses an action $a(s)$ from the action set $\mathcal{A}_{s}$ based on the current state $s$. The allocation decision must satisfy the finite energy capacitor constraint as $n \leq N \leq K_{\max }$ and the finite data buffer constraint as $w \leq W \leq D_{\max }$, where $n \in 0,1, \cdots, N$ and $w \in 0,1, \cdots, W$, respectively.

Let $P_{e}(\gamma)$ denote the instantaneous BER with received SNR $\gamma$. An approximate expression of BER for M-QAM is given in [100] and it can be expressed by,

$$
P_{e}(\gamma)=\frac{2}{v}\left(1-\frac{1}{\sqrt{M}}\right) \sum_{i=1}^{\left\lceil\frac{\sqrt{M}}{2}\right\rceil} \operatorname{erfc}\left((2 i-1) \sqrt{\frac{3 v \gamma P_{T}}{2(M-1) \bar{P}}}\right)
$$

where $v=\log _{2}(M)$ is the number of bits that modulates a $2^{v}$-QAM symbol and $P_{T}$ is the normalized power allocation and corresponds one-to-one to the number of EUs that are allocated. To obtain the optimum cross-layer adaptive transmission policy, a complete mapping from the states to the actions that maximize the sum of all long-term expected rewards for each state $s$ must be found, taking into account the system constraints, i.e., buffer delay and packet overflow. 


\section{Reward Model}

The choice for an action in a particular state is selected by the associated costs. The controller chooses the action that results in maximizing the reward (lowest cost). An action in a particular state results in a determined cost for each objective to be gained. The prime objective in this chapter is to maximize the network throughput for an online pointto-point EH communication system while minimizing queueing delay and buffer overflow costs so that they are within tolerable limits as well as satisfying the EH constraints. A cost function $R(s, a)$ constitutes the relationship between the state-action pair $(s, a)$ and the system reward. Given a system state and a corresponding action, the system reward (also called associated cost) can be represented as

$$
R(s, a)=Q(s, a)-G(s, a)
$$

where $Q(s, a)$ refers to the instant income and cost that are obtained by taking an action $a(s)$ at state $s$. Each objective (both the main objective and constraint objective) with a cost function is described as follows:

1) Adaptive Modulation Rate: EH power sources are different than conventional power sources in terms of their renewable feature. Unlike conventional wireless networks, which operate with battery of non-renewable and limited energy, wireless networks with EH capability have renewable feature. However, although transmitting with minimum power is significant in wireless devices that usually operate with conventional power sources, optimally controlling the transmitting power in EH technology, even by utilizing more power under certain conditions (necessity and availability), is desirable. Based on the system states, i.e., channel, battery, and data buffer, the scheduler determines how many EUs should be allocated for data transmission, and consequently the number of $w$ packets that are taken from the data buffer for transmission. Hence, 
the adaptive modulation rate is equivalent to the immediate system reward and can be expressed as:

$$
Q_{E}(s, a)=w, s \in \mathcal{S}, a \in \mathcal{A}
$$

2) Buffer Overflow Cost: It can be noted that while the scheduler optimally controls the transmission power and the adaptive rate, some incoming packets may be dropped due to insufficient space in the data buffer. Therefore, packet overflow rate is an important QoS requirement when the incoming traffic is bursty and the buffer is limited in size. The buffer overflow rate cost depends on the current buffer occupancy, arrival packets and the number of transmitted packets. When the buffer is full, the probability of dropped packets is high. Suppose, the current buffer state is $d$ and the controller takes $w$ packets from the buffer. Then, the buffer can accommodate $\left(x=D_{\max }-d+w\right)$ arriving packets. Now, if the arriving packets $f$ are larger than $x,(f-x)$ the packets will be dropped. Therefore, the immediate overflow cost $Q_{O}(s, a)$ by taking an action $a(s)$ at state $s$ is given by the number of packets that are dropped from the buffer as a result of insufficient storage and it can be expressed as

$$
Q_{O}(s, a)=\varphi\left(f,\left(D_{\max }-d+w\right)\right), s \in \mathcal{S}, a \in \mathcal{A}
$$

where $\varphi(y, z)$ is a positive difference function, which returns the difference of $y$ and $z$ when $y>z$, and it returns 0 when $y \leq z$.

3) Queueing Delay Cost: Since many wireless network applications are delay sensitive, delay is another important parameter that quantifies QoS requirements in modern wireless networks. Based on the application of QoS requirements, the maximum tolerable queueing delay is determined. For example, real-time traffic has less tolerance in latency compared to the best-effort traffic. The queueing delay cost $Q_{D}(s, a)$ for action $a(s)$ at state $s$ is the ratio of the corresponding number of packets in the buffer 
$d$ in state $s$, and the average packet arrival rate $\left(\lambda_{c}\right)$, as follows:

$$
Q_{D}(s, a)=\frac{d}{\lambda_{c}}, s \in \mathcal{S}, a \in \mathcal{A}
$$

The expected system cost $G(s, a)$, on the other hand, is defined as follows:

$$
G(s, a)=C(s, a) \tau(s, a),
$$

where $\tau(s, a)$ is the expected service time, which will be discussed below. $C(s, a)$ is the cost rate of the service time when an action $a(s)$ is selected. $C(s, a)$ can be determined by the power allocation at state $s$, shown as

$$
C(s, a)=P_{T},
$$

where the transmission power $P_{T}$ can be calculated using (5.6) with instantaneous received SNR $\gamma$ replaced by the average received SNR $\bar{\gamma}$ from the following equation: $\bar{\gamma}=$ $\frac{1}{\pi_{j}^{c}} \int_{\gamma_{j-1}}^{\gamma_{j}} f_{\Gamma}(\gamma) d \gamma$, which is equalvelant to the number of allocated EUs; $\xi(n)$.

\section{Sojourn Time}

The average expected time $\tau(s, a)$ is the time duration from the current event to others after selecting action $a(s)$. The average rate of events $\gamma(s, a)$, consequently, is the sum of all elements processes' rates from state $s$ to other states after selecting action $a(s) \cdot \gamma(s, a)$ and $\tau(s, a)$ can be computed as follows:

$$
\gamma(s, a)=\tau(s, a)^{-1}= \begin{cases}\lambda_{c}+\lambda_{e}, & \tilde{e} \in\{F, G\} \quad \text { or } \quad \tilde{e} \in\left\{C_{c}\right\}, a=0, \\ \lambda_{c}+\lambda_{e}+\frac{\mu_{c}}{\xi(n)}, & \tilde{e} \in\left\{C_{c}\right\}, a=n .\end{cases}
$$


where $R$ is the modulation rate that is adapted by occupying $n \mathrm{EU}$ at channel state $c$, and $a(s)=-1$ represents either harvesting new EUs or receiving new packets in the data buffer. In both cases, no action is required except that the transmitter's battery and data buffer are updated. When harvesting new EUs $(\tilde{e} \in\{F\})$ or new packets are arriving at the transmitter's data buffer $(\tilde{e} \in\{G\})$, no action is required and none of the ongoing services are being processed. Once the channel state is changed $\left(\tilde{e} \in\left\{C_{c}\right\}\right)$, the scheduler determines the system state and the decision action is taken.

\section{Transition Probability}

Next, the state transition probability from state $s$ to state $s^{\prime}$ when an action $a(s)$ is selected is derived, which is denoted as $P\left(s^{\prime} \mid s, a\right)$, under different events $\tilde{e} \in\left\{G, F, C_{c}\right\}$.

- For the given state $s=\left(s_{n, w}, K, D, C_{c}\right)$, the transition probability $P\left(s^{\prime} \mid s, a\right)$ to the next state $s^{\prime}$ can be obtained as

$$
P\left(s^{\prime} \mid s, a\right)= \begin{cases}\frac{\mu_{c}}{\xi(n) \gamma(s, a)}, & a=n, s^{\prime}=\left(s_{n, w}, K-n, D-w, C_{c}\right) \\ \frac{\lambda_{e}}{\gamma(s, a)}, & a=n, s^{\prime}=\left(s_{n, w}, K-n+1, D-w, G\right) \\ \frac{\lambda_{c}}{\gamma(s, a)}, & a=n, s^{\prime}=\left(s_{n, w}, K-n, D-w+1, F\right)\end{cases}
$$

where the modulation rate $R \in\{0,2,4,6\}$.

- For the given state $s=\left(s_{n, w}, K, D, G\right)$, the transition probability $P\left(s^{\prime} \mid s, a\right)$ to the next state $s^{\prime}$ can be obtained as

$$
P\left(s^{\prime} \mid s, a\right)= \begin{cases}\frac{\mu_{c}}{\xi(n) \gamma(s, a)}, & a=n, s^{\prime}=\left(s_{n, w}, K-n+1, D-w, C_{c}\right) \\ \frac{\lambda_{e}}{\gamma(s, a)}, & a=-1, s^{\prime}=\left(s_{n, w}, K+1, D, G\right) \\ \frac{\lambda_{c}}{\gamma(s, a)}, & a=-1, s^{\prime}=\left(s_{n, w}, K, D+1, F\right)\end{cases}
$$


- For the given state $s=\left(s_{n, w}, K, D, F\right)$, the transition probability $P\left(s^{\prime} \mid s, a\right)$ to the next state $s^{\prime}$ can be obtained as

$$
P\left(s^{\prime} \mid s, a\right)= \begin{cases}\frac{\mu_{c}}{\xi(n) \gamma(s, a)}, & a=n, s^{\prime}=\left(s_{n, w}, K-n, D-w, C_{c}\right) \\ \frac{\lambda_{e}}{\gamma(s, a)}, & a=-1, s^{\prime}=\left(s_{n, w}, K+1, D, G\right) \\ \frac{\lambda_{c}}{\gamma(s, a)}, & a=-1, s^{\prime}=\left(s_{n, w}, K, D+1, F\right)\end{cases}
$$

\subsubsection{Problem Formulation and Policy}

The objective is to make decisions at the beginning of the epoch so as to maximize the supportable data rate under average packet overflow and average delay constraints. The problem can be mathematically formulated as follows:

$$
\begin{array}{ll}
\max & Q_{E} \\
\text { s.t. } & Q_{D} \leq B_{d} \\
& Q_{O} \leq P_{o}
\end{array}
$$

where $B_{d}$ and $P_{o}$ are the allowable average delay threshold and average packet overflow rate, respectively. The actual value of $B_{d}$ is application dependent and known to the transmitter for the target application. On the other hand, a smaller (larger) $P_{o}$ represents a strict (loose) buffer overflow constraint.

Two adaptation policies are considered to investigate the cross-layer performance as follows:

1. Channel-Dependent Static Policy: The adaptive modulation rate is chosen based on channel state only but it maintains a fixed specified BER. However, this adaptation has a major drawback because it does not consider the finiteness of the data buffer or delay threshold, and consequently cannot guarantee any overflow or delay requirements. On 
the other hand, this policy is deterministic and the transition pattern of states only vary when there is a change in a channel state. Specifically, in this adaptation scheme, the channel will be divided into a finite $C=A$ number of states and the controller selects action $a_{i} \in \mathcal{A}$ when the gain of channel state $c_{i}$ places it in the range $\left[\gamma_{i-1}, \gamma_{i}\right)$.

2. Joint Battery, Buffer and Channel Dependent Dynamic Policy: The proposed adaptation policy is based on SMDP, and it takes into account the energy capacitor, data buffer occupancy and channel state to tackle overflow and delay in a purposeful direction. The controller considers changes rrelated to data buffer, battery, and channel to apply the proper action at each system state. This policy provides optimum power allocation and guarantees both packet overflow and buffering delay requirements.

\subsection{Solution Techniques}

We discuss the determination of the extended instant reward, which is based on the rule that determines the action for each specific state. The expected instant reward $R(s, a)$ during $\tau(s, a)$ is determined according to the discounted reward model in [93], as below:

$$
\begin{aligned}
R(s, a) & =Q(s, a)-C(s, a) E_{s}^{a}\left[\int_{0}^{\tau} e^{-\alpha t} d t\right] \\
& =Q(s, a)-C(s, a) E_{s}^{a}\left[\frac{\left[1-e^{-\alpha \tau}\right]}{\alpha}\right] \\
& =Q(s, a)-\frac{C(s, a)}{\alpha+\gamma(s, a)},
\end{aligned}
$$

where $Q(s, a)=\left[Q_{E}(s, a)-Q_{O}(s, a)-Q_{D}(s, a)\right]$ and $\alpha$ represents a continuous-time discounting factor. The policy for the channel-dependent adaptation scheme is static and unique since it is only dependent on the current measured channel state. However, this channel-dependent policy causes unavoidable queueing delay and packet overflow 
because the controller does not have any control over them. For the cross-layer adaptation scheme, the policy takes the battery occupancy and traffic behaviour into account to tackle delay and overflow in an object-oriented way. For example, the transmitter needs different transmission powers in different channel states since channel gains are different. However, the transmitter might also use a higher power level to keep the delay within limits, and to avoid packet overflow when the buffer is nearly full and/or when the average arrival rate is higher and vice versa. That is, it is assumed that the models for system state transition probabilities and cost functions for all the actions are known. Therefore, the transmitter can compute the optimal policy via dynamic programming algorithm using SMDP formulation. The formulation and its solution technique are discussed below:

\subsubsection{Cross-Layer and Policy Evaluation}

When the state transition probabilities for the system are known, the problem can be formulated as an SMDP problem and find the optimal policy using dynamic programming algorithm. In SMDP formulation, the models for system states, actions, transition probabilities and costs of the system are given. The problem formulated in Eq. (5.17) is solved using an unconstrained weighted sum of the three objectives $Q_{E}$, $Q_{O}$ and $Q_{D}$, where they are maximized via dynamic programming algorithm (i.e., policy iteration algorithm). Specifically, our objective is to find the optimum decision so that the average weighted of all the costs $\left[Q(s, a)=Q_{E}(s, a)-\beta_{1} Q_{O}(s, a)-\beta_{2} Q_{D}(s, a)\right]$ is maximized. $\beta_{1}$ and $\beta_{2}$ are weighting factors that indicate the importance of a particular cost over others. Hence, each combination of the weighting factors determines a unique optimal policy for the adaptation problem because of the unique cost function. By finding a complete mapping from the states to the actions, the optimal resource allocation policy can be obtained. Based on the above transition probabilities and the reward model in Eq. (5.18), the maximum long-term discounted reward of the state $s$ 
for our approach can be formulated as the discount reward model defined in a Bellman equation as follows:

$$
v(s)=\max _{a \in \mathcal{A}}\left[R(s, a)+\lambda \sum_{s^{\prime} \in \mathcal{S}} p\left(s^{\prime} \mid s, a\right) v\left(s^{\prime}\right)\right],
$$

where $\lambda=\frac{\gamma(s, a)}{\alpha+\gamma(s, a)}<1$ to ensure the convergence of the calculation. In addition, the state transition probability after uniformization $\tilde{p}\left(s^{\prime} \mid s, a\right)$ can be expressed as

$$
\tilde{p}\left(s^{\prime} \mid s, a\right)= \begin{cases}1-\frac{\left[1-p\left(s^{\prime} \mid s, a\right)\right] \gamma(s, a)}{L}, & s^{\prime}=s \\ \frac{p\left(s^{\prime} \mid s, a\right) \gamma(s, a)}{L}, & s^{\prime} \neq s\end{cases}
$$

where $L=\lambda_{c}+\lambda_{e}+\frac{\mu_{c}}{\xi(n)}$.

The expected real-time reward must be unitized in order to make uniform the continuoustime Markov decision process. The normalized long-term expected reward $\tilde{v}(s)$ for the proposed adaptive optimal data rate for the cross-layer EH network is stated as follows

$$
\tilde{v}(s)=\max _{a \in \mathcal{A}}\left[\tilde{R}(s, a)+\tilde{\lambda} \sum_{s^{\prime} \in S} \tilde{p}\left(s^{\prime} \mid s, a\right) \tilde{v}\left(s^{\prime}\right)\right]
$$

where the unitized reward function $\tilde{R}(s, a)$ and the unitized parameter $\tilde{\lambda}$ are determined as follows

$$
\begin{gathered}
\tilde{R}(s, a)=R(s, a) \frac{\alpha+\gamma(s, a)}{\alpha+L} \\
\tilde{\lambda}=\frac{L}{(L+\alpha)}
\end{gathered}
$$

The objective of determining $\tilde{v}(s)$ is to choose an action that maximizes the right hand side expression. Then, the optimal action (policy) can be obtained by solving $\left(P_{\text {opt }}\right)$ 
at each state $s$, which can be calculated as

$$
P_{\text {opt }}(s)=\arg \max _{a \in \mathcal{A}}\left[\tilde{R}(s, a)+\tilde{\lambda} \sum_{s^{\prime} \in S} \tilde{p}\left(s^{\prime} \mid s, a\right) \tilde{v}\left(s^{\prime}\right)\right],
$$

The optimal policy can be obtained by applying an iteration approach [91] as summarized in Algorithm 4. In the beginning, both $\tilde{v}(s)$ and $P_{\text {opt }}(s)$ are initiated to be zero

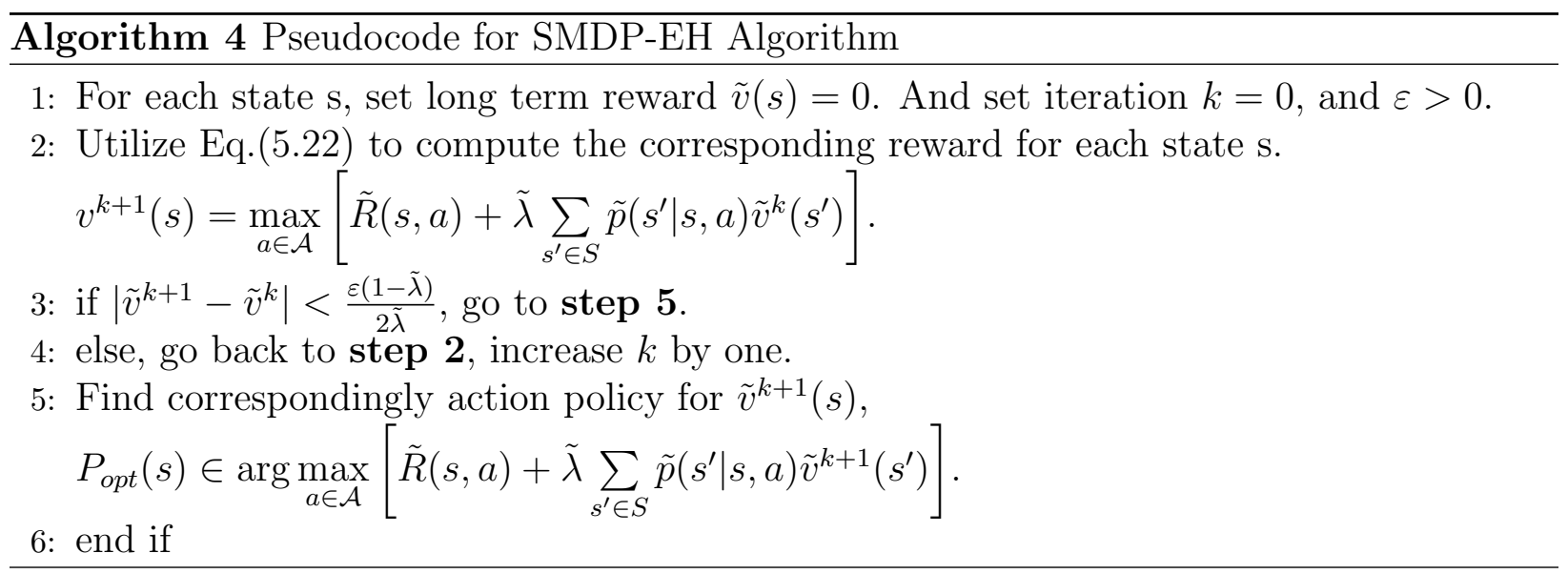

for each state $s$. For each state $s, \tilde{v}(s)$ and $P_{\text {opt }}(s)$ are repeatedly calculated until the value of $\tilde{v}(s)$ for every state $s$ equals the one of related $\tilde{v}^{\prime}(s)$ in the previous iteration, which indicates that the convergence is reached. The output $P_{\text {opt }}(s)$ for all states is the decision policy of the system, which results in obtaining the maximum discounted reward.

\subsection{Performance Evaluation}

This section shows the performance of two adaptation policies. The parameter values are set as follows: it is assumed energy harvesting rate and packet arrival rate are following a Poisson distribution with an average rate $\left(\lambda_{e}=2\right)$ and $\left(\lambda_{c}=3\right)$, respectively. In addition, it is assumed that the finite energy capacitor $K_{\max }=20$, finite 
data buffer $D_{\max }=20, N_{s} / N_{p}=1$, and the number of channel states and actions are $C=4$ and $U=4$, respectively. We assume i.i.d. Rayleigh fading channels, where the gain $h$ follows a Rayleigh distribution with mean $(\bar{m}=1)$ and average transmission power $(\bar{P}=1 \mathrm{~mW})$ and corresponding normalized average received SNR $(\bar{\gamma}=1)$, average channel BER $\left(\bar{P}_{e}=10^{-4}\right)$ and the modulation constellation set $w=[0,2,4,6]$ bits/symbol.

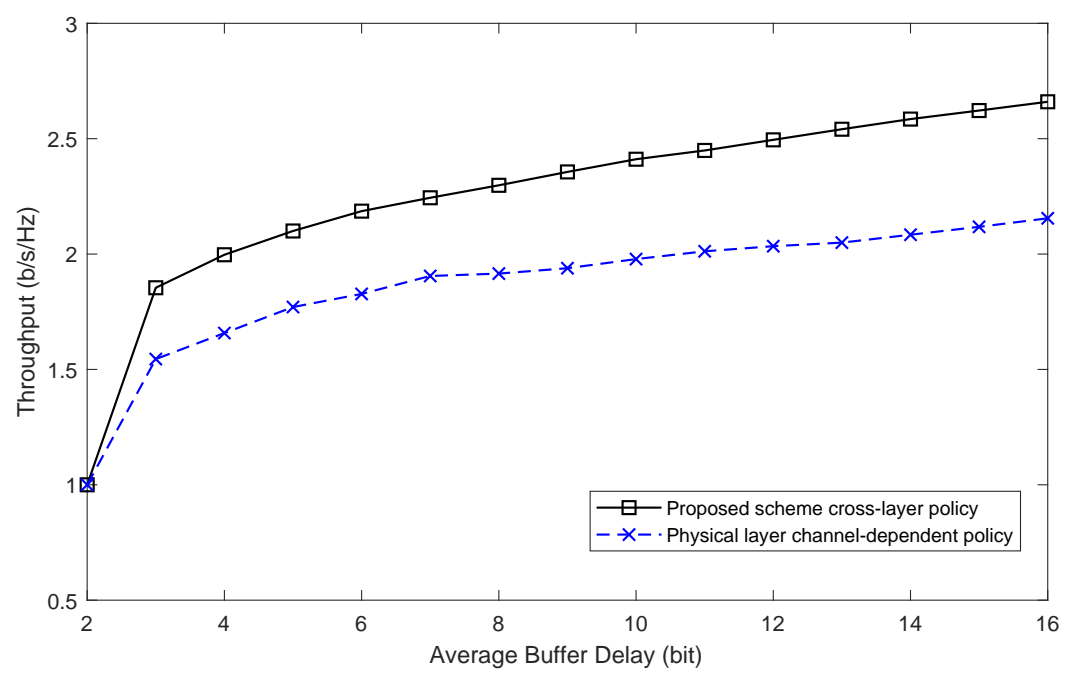

Figure 5.2: Effect of average buffer delay on the throughput among different schemes.

Fig.5.2 shows the maximum supportable throughput-maximum buffer trade-off curve for the cross-layer dynamic and single layer static policies. It is noticeable that the throughput increases with an increase in the average buffer delay for both policies. However, the throughput increasing rate is high for smaller values of data buffer size, while the throughput increasing rate slows down when buffer size increases. Fig.5.2 also shows that the proposed scheme outperforms the static policy and performance gap between them increases with increasing the average buffer delay requirement. The reason behind that the single layer static policy does not keep track of the battery and buffer states when taking an action and yields in less performance by consuming more energy that the battery contains while our proposed policy keeps track of both the 
battery and data buffer states, and takes optimal decision actions in each state. Hence, it can be concluded that despite the higher complexity of the proposed scheme, it is still worthwhile to implement the cross-layer dynamic scheme because of the enormous improvement in the performance between the two schemes, especially when $B_{d} \geq 3$.
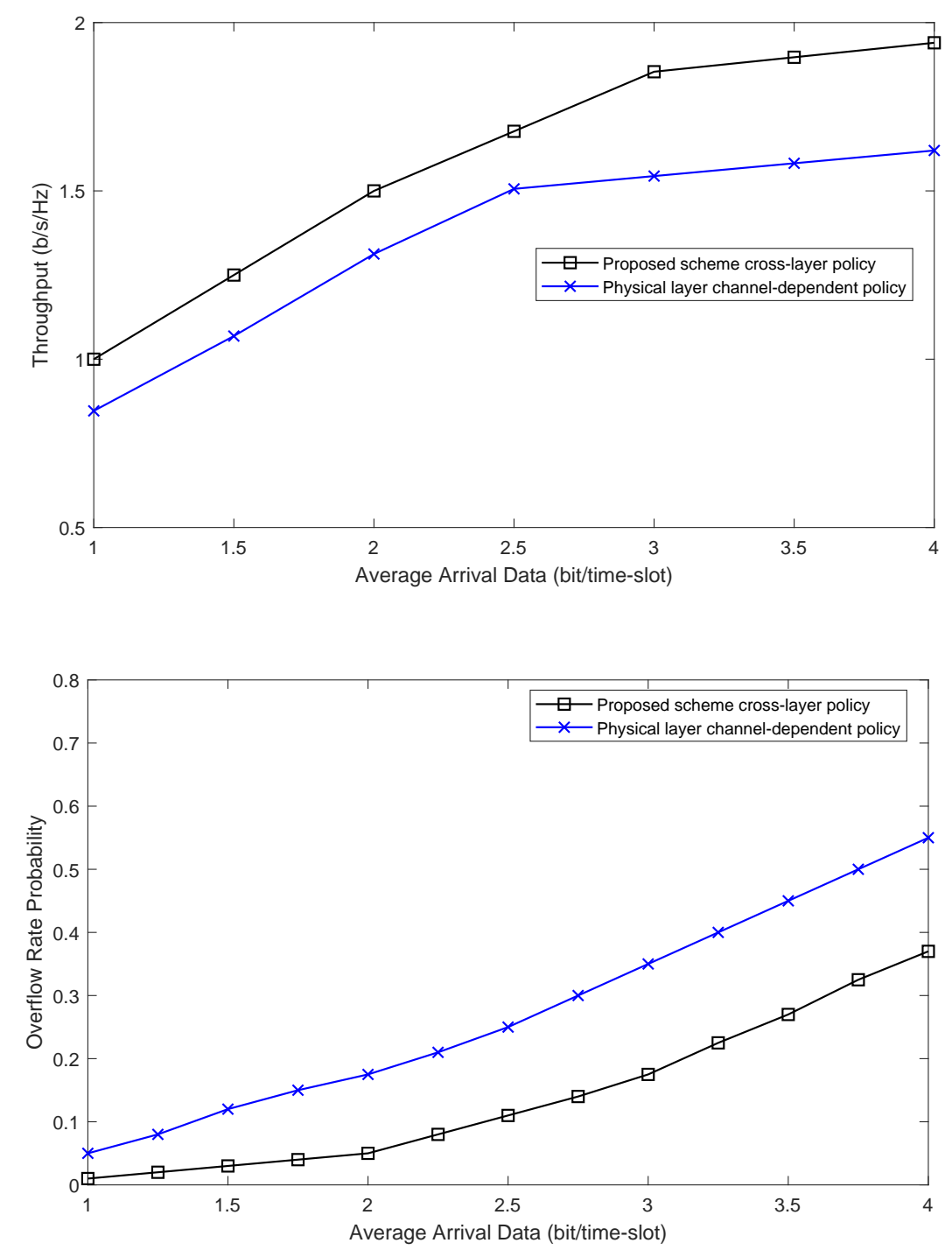

Figure 5.3: Effect of average packet arrival rate on the throughput and the overflow probability rate among different schemes.

We compare the throughput and the blocking probability of the physical-layer static 
adaptation policy with the cross-layer dynamic adaptation policy when the average buffer delay $\left(B_{d}=3\right)$ in Fig. 5.3. It illustrates that the average throughput of the cross-layer dynamic scheme outperforms the channel-dependent static policy for all packet arrival rates. It is clear that the performance of the channel-dependent static policy degrades by increasing data arrival rates. Fig.5.3 also displays how the blocking probability grows with the increase of the packet arrival rates in channel-dependent static policy while the blocking rate is low in cross-layer dynamic policy even with the increase in the data arrival rate. The reason is that the scheduler in channel-dependent policy applies different modulation constellations based on channel state only without tracking the capacitor and buffer states. Therefore, the policy has no guarantee with respect to the overflow requirement, consequently throughput. In cross-layer policy, on the other hand, BER as well as packet overflow requirements are guaranteed for increased data arrival rates.

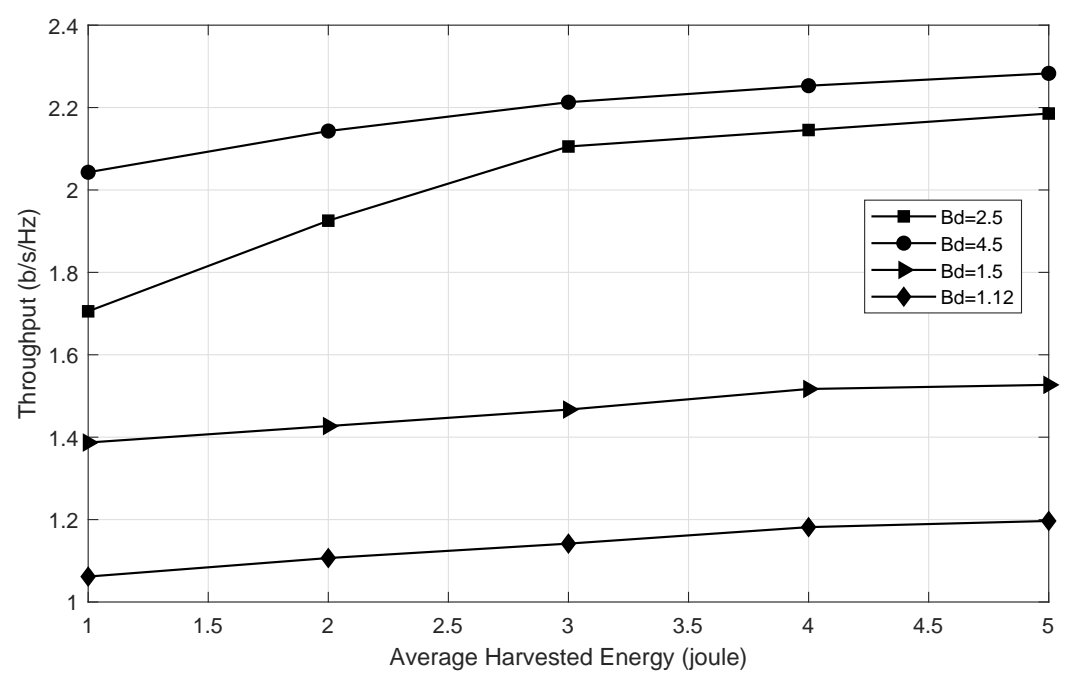

Figure 5.4: Effect of average harvested energy on the throughput for the proposed scheme among various average buffer delays.

The effect of the average harvested energy on the throughput for the proposed scheme for different values of maximum allowable average delay $B_{d}=1.12,1.5,2.5,4.5$ packets 
is shown in Fig.5.4. The throughput increases with an increase of average harvested energy for a given $B_{d}$. Although the higher available energy supports the network to transmit more data, the increasing rate is comparatively less when there is a strict delay constraint. For instance, in the case of $B_{d}=1.12$, i.e., when a system can tolerate fewer delays, the throughput can be increased by $0.14 \mathrm{~b} / \mathrm{s} / \mathrm{Hz}$ if the average harvested energy is increased from $1 \mathrm{~J} / \mathrm{s}$ to $5 \mathrm{~J} / \mathrm{s}$. In contrast, with the same incremental harvested energy, the throughput is improved by $0.48 \mathrm{~b} / \mathrm{s} / \mathrm{Hz}$ in the case of $B_{d}=2.5$, i.e., when a system can tolerate comparatively more delay.

\subsection{Chapter Summary}

In this Chapter, an SMDP framework has been utilized to determine the optimal policy of a cross-layer design for an online point-to-point EH communication system based on channel-dependent static adaptation and cross-layer dynamic adaptation. In cross-layer adaptation, throughput is maximized by tracking battery, data buffer, and channel states to optimally control the transmission power and rate over the transmission time intervals. Numerical results illustrated that cross-layer adaptation policy outperforms channel-dependent policy by guaranteeing overflow rate, and hence, network throughput in a green radio network with EH sources. Our proposed cross-layer scheme has been shown to be implementable compared to the benchmark scheme, and it still achieves almost the same throughput as the benchmark scheme. 


\section{Chapter 6}

\section{Conclusions}

\subsection{Conclusion}

In this thesis, we investigated the design of optimal transmission policies for energy harvesting communication systems. Resource allocation was considered for point-to-

point and two-hop relay networks with an energy harvesting source. The main results of each chapter are summarized as follows:

In Chapter 3, An efficient transmission policy by maximizing the network throughput has been investigated for the energy harvesting two-hop delay-tolerant and non-delaytolerant relay networks in an offline setting. It was assumed that the information on energy harvesting as well as channel fading status was available prior to the beginning of the transmission. Specifically, the simple and elegant RGWF approach that solves the power allocation problem was extended numerically into a simulation representation. The importance of this representation is that it provides more insight into the problems and the solutions such that various wireless systems can be analysed. The advantage of adapting the RGWF algorithm for the throughput maximization problem under a Rayleigh fading channel is demonstrated. For a two-hop communication system, two 
schemes were proposed that maximize the network performance from a throughput perspective for both delay tolerant and non-delay tolerant networks. The transmission scheduling time has been derived for the source and the relay based on the RGWFEH profile to obtain an efficient transmission policy. Numerical results illustrated that optimizing both transmission scheduling and power allocation result in higher throughput. Moreover, simulations show that the proposed approach is simple, efficient and provides significant guidelines on network deployment and resource management in a green radio network with $\mathrm{EH}$ sources.

Chapter 4 investigated the resource allocation problem for a point-to-point communication system with energy harvesting constraints over a fading channel. A more practical online setting was considered, where the energy arrival information and the channel state information is known causally. To overcome the high complexity and curse of dimensionality of the dynamic programming, which is the conventional approach for solving stochastic control problems, discrete dynamic programming was utilized by casting the optimization problem as a semi-Markov decision process (SMDP). In the SMDP formulation, the set of transmission power levels is discrete and finite. Moreover, the channels are quantized to a finite number of states. The quantizations of channel and power level result in a finitely-sized state space, and makes the SMDP formulation mathematically tractable to solve the throughput optimization problem in the online setting. Consequently, an optimal SMDP-based policy was proposed that is dynamically adaptive to the changing of the channel status and the varying of arriving harvested energy and data requests to making scheduling decisions regarding the energy allocation. It was shown that adapting the SMDP-based formulation results in maximizing the network throughput, reducing the service rejection probability and provides guidelines for resource management in green communication with $\mathrm{EH}$ transmitters.

In chapter 5, a cross-layer optimal adaptation transmission policy for a point-to-point 
communication system with EH constraints over a Rayleigh fading channel based on SMDP was investigated. Specifically, we studied the capability of employing the SMDP-based policy optimization for an adaptive cross-layer EH wireless communication system with the objective of maximizing network throughput while minimizing the dropping probability and data buffer delay. In cross-layer adaptation, throughput should be maximized by tracking battery, data buffer, and channel states to optimally control the transmission power and rate over the transmission time intervals. Hence, a novel framework based on the SMDP approach was formulated for the proposed system model with the objective of maximizing network throughput by optimally allocating the resource while maintaining minimum buffering delay and packet overflow. The proposed SMDP-based dynamic programming approach has proven to be dynamically adaptive to the change of the channel and/or buffers states that optimally satisfy the BER requirements at the physical layer, and the overflow requirements at the data-link layer. 
Appendix 1

Acronyms 


$\begin{array}{ll}\text { AWGN } & \text { Additive White Gaussian Noise } \\ \text { CSI } & \text { Channel State Information } \\ \text { CWF } & \text { Conventional Water-Filling } \\ \text { DP } & \text { Dynamic Programming } \\ \text { DS } & \text { Delay Sensitive } \\ \text { DT } & \text { Delay Tolerant } \\ \text { DWF } & \text { Directional Water-Filling } \\ \text { EH } & \text { Energy Harvesting } \\ \text { EHN } & \text { Energy Harvesting Network } \\ \text { ESI } & \text { energy state information } \\ \text { GR } & \text { Green Radio } \\ \text { GWF } & \text { Geometric Water-Filling } \\ \text { HU } & \text { Harvest-Use } \\ \text { HUS } & \text { Harvest-Use-Save } \\ \text { KKT } & \text { Karush-Kuhn-Tucker } \\ \text { MDP } & \text { Markov Decision Process } \\ \text { MIMO } & \text { Multiple Input, Multiple Output } \\ \text { PDDR } & \text { Pre-defined Data Rates } \\ \text { PSR } & \text { Power splitting-based relaying } \\ \text { QoS } & \text { Quality of Service } \\ \text { RF } & \text { Radio Frequency } \\ \text { RGWF } & \text { Recursive Geometric Water-Filling } \\ \text { RID-AC } & \text { Relay In Demand using Average Capacity } \\ \text { RID-AF } & \text { Relay In Demand using Average Fading } \\ \text { RIPE } & \text { Relay In Partial Epoch } \\ \text { RRM } & \text { Radio Resource Management } \\ \text { SMDP } & \text { Semi-Markov Decision Process } \\ \text { SNR } & \text { Signal-to-Noise Ratio } \\ \text { SWIPT } & \text { Simultaneous Wireless Information and Power Transfer } \\ \text { TSR } & \text { Time Switching-Based Relaying } \\ \text { WF } & \text { Water-Filling } \\ \text { WLAN } & \text { Wireless Local Area Network } \\ & \end{array}$




\section{References}

[1] International Energy Agency, "Energy technology scenarios and strategies for a more secure and sustainable energy future," International Energy Agency, Paris, June 2006.

[2] F. R. Yu, X. Zhang, and V. C. M. Leung, "Green communications and networking," New York: CRC Press, 2012.

[3] W. Ye, J. Heidemann, and D. Estrin, "An energy-efficient mac protocol for wireless sensor networks," Joint Conference of the IEEE Computer and Communications Societies, vol. 3, pp. 1567 - 1576, 2002.

[4] R. C. Shah and J. M. Rabaey, "Energy aware routing for low energy ad hoc sensor networks," IEEE Wireless Communications and Networking Conference (WCNC), vol. 1, pp. 350-355, June 2002.

[5] W. Vereecken, W. V. Heddeghem, D. Colle, M. Pickavet, and P. Demeester, "Overall ict footprint and green communication technologies," International Symposium on Communications, Control and Signal Processing, pp. 1-6, March 2010.

[6] European Comission, "Addressing the challenge of energy efficiency through information and communication technologies," May 2008. 
[7] A. Kansal, J. Hsu, S. Zahedi, and M. B. Srivastava, "Power management in energy harvesting sensor networks," ACM Transactions on Embedded Computing Systems (TECS), vol. 6, no. 4, Sept. 2007.

[8] C. Vigorito, D. Ganesan, and A. Barto, "Adaptive control of duty-cycling in energy harvesting wireless sensor networks," IEEE Communications Society Conference on Sensor, Mesh and Ad Hoc Communications and Networks, pp. 21-30, 2007.

[9] V. Sharma, U. Mukherji, V. Joseph, and S. Gupta, "Optimal energy management policies for energy harvesting sensor nodes," IEEE Transactions on Wireless Communications, vol. 9, no. 4, pp. 1326-1336, April 2010.

[10] Q. Zhang, A. Agbossou, Z. Feng, and M. Cosnier, "Solar micro-energy harvesting based on thermoelectric and latent heat effects part ii: Experimental analysis," Sensors and Actuators A: Physical, vol. 163, no. 1, pp. 284290, Sep. 2010.

[11] S. Sudevalayam and P. Kulkarni, "Energy harvesting sensor nodes: Survey and implications," IEEE Communications Surveys Tutorials, vol. 13, no. 3, pp. 443461, April 2011.

[12] C. K. Ho and R. Zhang, "Optimal energy allocation for wireless communications with energy harvesting constraints," IEEE Transactions on Signal Processing, vol. 60 , no. 9, pp. $4808-4818$, Sept. 2012.

[13] R. Rajesh, V. Sharma, and P. Viswanath, "Capacity of fading gaussian channel with an energy harvesting sensor node," IEEE Global Telecommunications Conference (GLOBECOM), pp. 1-6, December 2011.

[14] Q. Zhang, A. Agbossou, Z. Feng, and M. Cosnier, "Throughput optimal policies for energy harvesting wireless transmitters with non-ideal circuit power," IEEE Journal on Selected Areas in Communications, vol. 32, no. 2, pp. 322-332, Feb. 2014. 
[15] M. A. Antepli, E. U. Biyikoglu, and H. Erkal, "Optimal packet scheduling on an energy harvesting broadcast link," IEEE Journal on Selected Areas in Communications, vol. 29, no. 8, pp. 17211731, Sep. 2011.

[16] J. Yang and S. Ulukus, "Optimal packet scheduling in an energy harvesting communication system," IEEE Transaction on Communications, vol. 60, no. 1, pp. 220-230, Jan. 2012.

[17] O. Ozel, K. Tutuncuoglu, J. Yang, S. Ulukus, and A. Yener, "Transmission with energy harvesting nodes in fading wireless channels: Optimal policies," IEEE Journal on Selected Areas in Communications, vol. 29, no. 8, pp. 1732-1743, Sept. 2011.

[18] A. Sendonaris, E. Erkip, and B. Aazhang, "User cooperation diversity-part i: System description and part ii: Implementation aspects and performance analysis," IEEE Transaction on Communication, vol. 51, no. 11, pp. 19271948, Nov. 2003.

[19] J. Laneman, D. Tse, and G. Wornell, "Cooperative diversity in wireless networks: Efficient protocols and outage behavior," IEEE Transactions on Information Theory, vol. 50, no. 12, pp. 30623080, Dec. 2004.

[20] M. Andrews, K. Kumaran, K. Ramanan, A. Stolyar, P. Whiting, and R. Vijayakumar, "Providing quality of service over a shared wireless link," IEEE Communication Magazine, vol. 39, no. 2, pp. 150154, Feb. 2001.

[21] J. Chen, "Resource allocation for delay constrained wireless communications," PhD thesis, University College London, 2010.

[22] X. Zhang, W. Cheng, and H. Zhang, "Heterogeneous statistical qos provisioning over 5g mobile wireless networks," IEEE Network Magazine, vol. 28, no. 6, pp. 4653, Dec. 2014. 
[23] M. Agiwal, A. Roy, and N. Saxena, "Next generation 5g wireless networks: A comprehensive survey," IEEE Communications Surveys Tutorials, vol. 18, no. 3, pp. 16171655, Feb. 2016.

[24] J. Andrews, S. Buzzi, W. Choi, S. Hanly, A. Lozano, A. Soong, and J. Zhang, "What will 5g be?," IEEE Journal on Selected Areas in Communications, vol. 32, no. 6, pp. 10651082, June 2014.

[25] T. Y. Kheng, "Analysis, design and implementation of energy harvesting systems for wireless sensor nodes," Thesis: National University of Singapore, 2010.

[26] R. J. Vullers, R. Schaijk, H. J. Visser, J. Penders, and C. V. Hoof, "Energy harvesting for autonomous wireless sensor networks," IEEE Solid-State Circuits Magazine, vol. 2, no. 2, pp. 29-38, Spring 2010.

[27] V. Leonov, T. Torfs, P. Fiorini, and C. Van Hoof, "Thermoelectric converters of human warmth for self-powered wireless sensor nodes," IEEE Sensors Journal, vol. 7 , no. 5, pp. 650-657, 2007.

[28] Z. Wang, V. Leonov, P. Fiorini, and C. Van Hoof, "Realization of a wearable miniaturized thermoelectric generator for human body applications," Sensors and Actuators A: Physical, vol. 156, no. 1, pp. 95-102, 2009.

[29] X. Lu and S.-H. Yang, "Thermal energy harvesting for wsns," IEEE Conference on Systems Man and Cybernetics, vol. 163, no. 1, pp. 3045-3052, Sept. 2010.

[30] Z. A. Eu, H.-P. Tan, and W. K. G. Seah, "Wireless sensor networks powered by ambient energy harvesting: an empirical characterization," IEEE Conference on Communications, pp. 1-5, 2010.

[31] "Cebekit: Windlab junior c-0200 technical characteristics," [Online]. Available: http://fadisel.cat/docs/c-0200-ing.pd. 
[32] C. Park and P. H. Chou, "Ambimax: Autonomous energy harvesting platform for multisupply wireless sensor nodes," in Proceedings of the IEEE Sensor and Ad Hoc Communications and Networks, vol. 1, pp. 168177, Sept. 2006.

[33] Y. K. Tan and S. K. Panda, "Self-autonomous wireless sensor nodes with wind energy harvesting for remote sensing of wind-driven wildfire spread," IEEE Transactions on Instrumentation and Measurement, vol. 60, no. 4, pp. 1367-1377, April 2011.

[34] J. Taneja, J. Jeong, and D. Culler, "Design, modeling, and capacity planning for micro-solar power sensor networks," IEEE Conference on Information processing in sensor networks, pp. 407-418, 2008.

[35] T. Voigt, H. Ritter, and J. Schiller, "Utilizing solar power in wireless sensor networks," IEEE International Conference on Local Computer Networks, pp. 416-422, October 2003.

[36] D. Kruger, C. Buschmann, and S. Fischer, "Solar powered sensor network design and experimentation," International Symposium on Wireless Communication Systems, pp. 11-15, September 2009.

[37] M. Gorlatova, J. Sarik, M. Cong, G. Grebla, M. Cong, I. Kymissis, and G. Zussman, "Movers and shakers: Kinetic energy harvesting for the internet of things," IEEE Journal on Selected Areas in Communications, vol. 33, no. 8, pp. 16241639, Aug. 2015.

[38] C. Wei and X. Jing, "A comprehensive review on vibration energy harvesting: Modelling and realization," Renewable and Sustainable Energy Reviews, vol. 74, no. 1, pp. 1-18, Sept. 2017.

[39] M. Peigney and D. Siegert, "Piezoelectric energy harvesting from traffic-induced bridge vibrations,", Smart Materials and Structures, vol. 9, no. 22, 2013. 
[40] S. Scorcioni, A. Bertacchini, D. Dondi, L. Larcher, P. Pavan, and G. Mainardi, "A vibration-powered wireless system to enhance safety in agricultural machinery," IEEE Conference on Industrial Electronics Society, pp. 3510-3515, 2011.

[41] N. Nakashima, H. Nishimoto, Y. Kawahara, and T. Asami, "Human detection system based on sensing through harvesting concept," Internet of Things Conference, 2010.

[42] N. S. Shenck and J. A. Paradiso, "Energy scavenging with shoe-mounted piezoelectrics," IEEE Micro, vol. 21, no. 3, pp. 30-42, May 2001.

[43] T. Y. Kheng, "Analysis, design and implementation of energy harvesting systems for wireless sensor nodes," Thesis: National University of Singapore, 2010.

[44] H. Nishimoto, Y. Kawahara, and T. Asami, "Prototype implementation of ambient rf energy harvesting wireless sensor networks," IEEE Sensors, p. 12821287, 2010.

[45] H. J. Visser, A. C. Reniers, and J. A. Theeuwes, "Ambient rf energy scavenging: Gsm and wlan power density measurements," IEEE Conference on Microwave, vol. 163, no. 1, pp. 721-724, Sept. 2008.

[46] A. N. Parks, A. P. Sample, Y. Zhao, and J. R. Smith, "A wireless sensing platform utilizing ambient rf energy," IEEE Conference on Biomedical Wireless Technologies, Networks, and Sensing Systems, pp. 154-156, 2013.

[47] T. Le, K. Mayaram, and T. Fiez, "Efficient far-field radio frequency energy harvesting for passively powered sensor networks," IEEE Journal of Solid-State Circuits, vol. 43, no. 5, pp. 1287-1302, Sept. 2008.

[48] U. Baroudi, S. Mekid, and A. Bouhraoua, "Radio frequency energy harvesting characterization: an experimental study," IEEE Conference in Trust, Security and Privacy in Computing and Communications, pp. 1976-1981, 2012. 
[49] H. J. Visser and R. J. Vullers, "Rf energy harvesting and transport for wireless sensor network applications: Principles and requirements," in Proceedings of the IEEE, vol. 101, no. 6, pp. 1410-1423, Sept. 2013.

[50] S. A. Bhalerao, A. V. Chaudhary, R. B. Deshmukh, and R. M. Patrikar, "Powering wireless sensor nodes using ambient rf energy," IEEE International conference on systems, man and cybernetics, vol. 9, no. 4, pp. 2695-2700, April 2006.

[51] J. A. Paradiso and T. Starner, "Energy scavenging for mobile and wireless electronics," IEEE Pervasive Computing, vol. 4, no. 1, pp. 18-27, Jan. 2005.

[52] R. Zhang and C. K. Ho, "Mimo broadcasting for simultaneous wireless information and power transfer," IEEE Transactions on Wireless Communications, vol. 12, no. 5, pp. 1989-2001, 2013.

[53] X. Zhou, R. Zhang, and C. K. Ho, "Wireless information and power transfer: Architecture design and rate-energy trade off," IEEE Global Communications Conference (Globecom), pp. 3982-3987, December 2012.

[54] I. Krikidis, S. Timotheou, and S. Sasaki, "Rf energy transfer for cooperative networks: Data relaying or energy harvesting?," IEEE Communication Letter, vol. 16, no. 11, pp. 1772-1775, Nov. 2012.

[55] J. Yang and S. Ulukus, "Transmission completion time minimization in an energy harvesting system," IEEE transactions on Communications, vol. 99, no. 9, pp. 1-10, Sept. 2013.

[56] P. He, L. Zhao, S. Zhou, and Z. Niu, "Recursive water-filling for wireless links with energy harvesting transmitters," IEEE transaction on vehicular technology, vol. 63, no. 3, pp. 1232 - 1241, March 2014.

[57] O. Ozel and S. Ulukus, "On the capacity region of the gaussian mac with batteryless energy harvesting transmitters," IEEE Global Communications Conference (GLOBECOM), pp. 2385-2390, 2012. 
[58] J. A. Paradiso and M. Feldmeier, "A compact, wireless, self powered pushbutton controller," International Conference on Ubiquitous Computing, pp. 299-304, October 2001.

[59] I. Krikidis, G. Zheng, and B. Ottersten, "Harvest use cooperative networks with half/full-duplex relaying," IEEE Wireless Communications and Networking Conference (WCNC), pp. 4256-4260, 2013.

[60] V. Raghunathan, S. Ganeriwal, and M. Srivastava, "Emerging techniques for long lived wireless sensor networks," IEEE Communications Magazine, vol. 44, no. 4, pp. 108-114, April 2006.

[61] L. R. Varshney, "Transporting information and energy simultaneously," EEE International Symposium on Information Theory, pp. 1612-1616, July 2008.

[62] D. S. Michalopoulos, H. A. Suraweera, and R. Schober, "Relay selection for simultaneous information transmission and wireless energy transfer: A trade-off perspective," IEEE Journal on Selected Areas in Communications, vol. 33, no. 8, pp. 1578-1594, Aug. 2015.

[63] A. A. Nasir, X. Zhou, S. Durrani, and R. A. Kennedy, "Relaying protocols for wireless energy harvesting and information processing," IEEE Transactions on Wireless Communications, vol. 12, no. 7, pp. 1536-1576, July 2013.

[64] H. Kawabata and K. Ishibashi, "Optimal energy management policies for energy harvesting sensor nodes," IEEE International Symposium on Personal, Indoor, and Mobile Radio Communication (PIMRC), Sept. 2014.

[65] V. Sharma, U. Mukherji, V. Joseph, and S. Gupta, "Trading wireless information and power transfer: Relay selection to minimize the outage probability," IEEE Global Conference on Signal and Information Processing (GlobalSIP), pp. 253257, Dec. 2014. 
[66] I. Ahmed, A. Ikhlef, R. Schober, and R. K. Mallik, "Joint power allocation and relay selection in energy harvesting af relay systems," IEEE Transactions on Wireless Communications, vol. 2, pp. 239-242, April 2013.

[67] C. Huang, R. Zhang, and S. Cui, "Optimal power allocation for outage probability minimization in fading channels with energy harvesting constraints," IEEE Transactions on Wireless Communications, vol. 13, no. 2, pp. 1074-1087, Feb. 2014.

[68] O. Orhan and E. Erkip, "Energy harvesting two-hop networks: optimal policies for the multi-energy arrival case," IEEE Sarnoff Symposium, pp. 1-6, 2012.

[69] D. Gunduz and B. Devillers, "Two-hop communication with energy harvesting," IEEE International Workshop on Computational Advances in Multi-Sensor Adaptive Processing (CAMSAP), pp. 201 - 204, 2011.

[70] C. Huang, R. Zhang, and S. Cui, "Throughput maximization for the gaussian relay channel with energy harvesting constraints," IEEE Journal on Selected Areas in Communications, vol. 31, no. 8, pp. 14691479, Aug. 2013.

[71] I. Ahmed, A. Ikhlef, R. Schober, and R. K. Mallik, "Power allocation for conventional and buffer-aided link adaptive relaying systems with energy harvesting nodes," IEEE Transactions on Wireless Communication, vol. 13, no. 3, pp. 11821195, March 2014.

[72] Y. Luo, J. Zhang, and K. B. Letaief, "Optimal scheduling and power allocation for two-hop energy harvesting communication systems," IEEE Transactions on Wireless Communications, vol. 12, no. 9, pp. 4729 - 4741, Sept. 2013.

[73] D. P. Bertsekas, "Dynamic programming and optimal control," Belmont, MA: Athens Scientific, vol. 1, 1995.

[74] R. Berry, E. Modiano, and M. Zafer, "Energy-efficient scheduling under delay constraints for wireless networks," Morgan Claypool, 2012. 
[75] O. Orhan and E. Erkip, "Optimal transmission policies for energy harvesting two-hop networks," Conference on Information Sciences and Systems (CISS), pp. 1-6, 2012.

[76] Q. Wu, M. Tao, W. Chen, and J. Wu, "Optimal energy-efficient transmission for fading channels with an energy harvesting transmitter," IEEE Global Communications Conference (GLOBECOM), pp. 4294-4299, 2014.

[77] J. Tang and X. Zhang, "Qos-driven power and rate adaptation over wireless links," IEEE Transaction Wireless Communication, vol. 6, no. 8, pp. 30583068, Aug. 2007.

[78] S. Ko and S. Kim, "Block waterfilling with power borrowing for multicarrier communications," IEEE Vehicular Technology Conference (VTC), pp. 1 - 5, Sept. 2008.

[79] J. Peng, G. Wei, and J. Zhu, "Power allocation method for ofdm system with both total and per-antenna power constraints," IEEE Communications Letters, vol. 12 , pp. $621-623,2008$.

[80] D. Zhao, Z. Fei, S. Li, and J. Kuang, "Improved iterative water-filling algorithm in mumimo system," IET International Conference on Wireless, Mobile and Multimedia Networks, pp. 173 - 178, 2010.

[81] P. He, L. Zhao, S. Zhou, and Z. Niu, "Water-filling: A geometric approach and its application to solve generalized radio resource allocation problems," IEEE Transactions on Wireless Communications, vol. 12, no. 7, pp. 3637 - 3647, 2013.

[82] M. Baljon and L. Zhao, "Resource allocation for two-hop communication with energy harvesting constraints," IEEE International Conference on Wireless Communications Signal Processing (WCSP), pp. 1-5, Oct. 2015.

[83] M. Baljon and L. Zhao, "Resource allocation for wireless networks with energy harvesting constraints over fading channels," Journal Issue on Information and 
Communication Technology, vol. 2, no. 1, pp. 9-19, Aug. 2016.

[84] S. Boyd and L. Vandenberghe, Convex optimization, Cambridge university press, 2004.

[85] A. Wachter and L. T. Biegler, "On the implementation of an interior-point filter line-search algorithm for large-scale nonlinear programming," Mathematical Programming, pp. 25-57, 2006.

[86] R. Fletcher and S. Leyffer, "Nonlinear programming without a penalty function," Mathematical Programming, vol. 91, no. 2, pp. 239-269, 2002.

[87] "Apopt, an exact solver for minlps.," Available: https://apopt.com.

[88] M. Baljon, M. Li, H. Liang, and L. Zhao, "Smdp-based resource allocation for wireless networks with energy harvesting constraints," IEEE Vehicular Technology Conference (VTC), pp. 1-6, Sep. 2017.

[89] H. Zhang, S. Huang, C. Jiang, K. Long, V. C. M. Leung, and H. Vincent Poor, "Energy efficient user association and power allocation in millimeter wave based ultra dense networks with energy harvesting base stations," IEEE Journal on Selected Areas in Communications, vol. 9, no. 35, pp. 1936-1947, Sept. 2017.

[90] L. Cai, Y. Liu, H. Luan, X. Shen, J. Mark, and H. Poor, "Sustainability analysis and resource management of wireless mesh net.," IEEE Journal on Selected Areas in Communications, vol. 32, no. 2, pp. 345-355, Feb. 2014.

[91] D. Bertsekas, "Approximate policy iteration: a survey and some new methods," Journal of Control Theory and Applications, vol. 9, no. 3, pp. 310-335, 2011.

[92] S. Mine and M. Puterman, "Markovian decision process," Elsevier, 1970.

[93] M. Puterman, "Markov decision processes: Discrete stochastic dynamic programming," Wiley, 2005.

[94] C. Thomas, H. Cormen, R. L. Rivest, and C. Stein, Introduction to algorithms, 2009. 
[95] H. Liang, L. X. Cai, D. Huang, X. Shen, and D. Peng, "An smdp-based service model for interdomain resource allocation in mobile cloud networks," IEEE Transactions on Vehicular Technology, vol. 61, no. 5, pp. 2222-2232, June 2012.

[96] Y. Liu, M. J. Lee, and Y. Zheng, "Adaptive multi-resource allocation for cloudletbased mobile cloud computing system," IEEE Transactions on Mobile Computing, vol. 15, no. 10, pp. 2398-2410, Oct 2016.

[97] K. Zheng, H. Meng, P. Chatzimisios, L. Lei, and X. Shen, "An smdp-based resource allocation in vehicular cloud computing systems," IEEE Transactions on Industrial Electronics, vol. 62, no. 12, pp. 7920-7928, Dec. 2015.

[98] H. He, H. Shan, A. Huang, and L. Sun, "Smdp-based resource allocation for video streaming in cognitive vehicular networks," IEEE International Conference on Communications in China (ICCC), pp. 1-6, 2015.

[99] J. G. Proakis, "Digital communications," Sensors and Actuators A: Physical, 2000.

[100] J. Lu, K. B. Letaief, J. C.-I. Chuang, and M. L. Liou, "M-psk and m-qam ber computation using signal-space concepts," IEEE Transactions on Communications, vol. 47, no. 2, pp. 181184, Feb. 1999. 
REFERENCES 